\title{
Machine Learning Aided Design of Conformal Cooling \\ Channels for Injection Molding
}

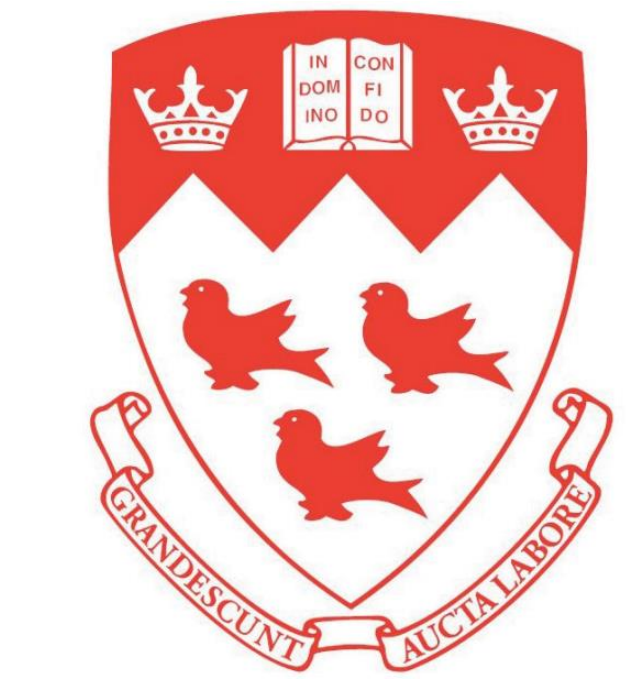

\author{
Zhenyang Gao \\ Department of Mechanical Engineering
}

Faculty of Engineering

McGill University, Montreal

April 2020

A thesis submitted to McGill University in partial fulfillment of the requirements of the degree of Master of Science

CZZhenyang Gao 2020 


\section{TABLE OF CONTENTS}

LIST OF FIGURES iv

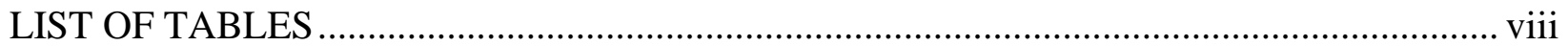

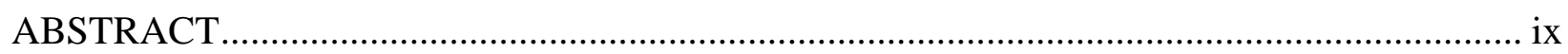

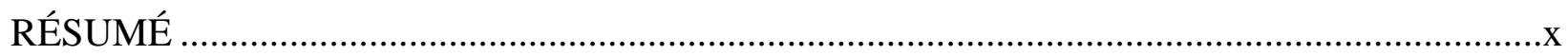

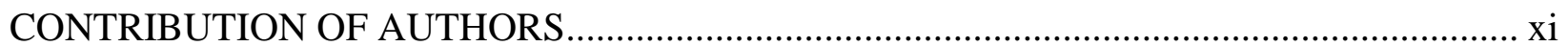

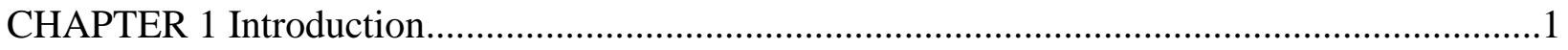

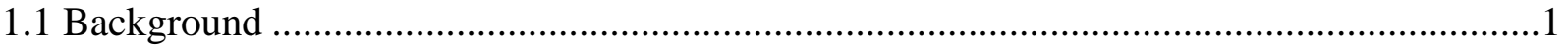

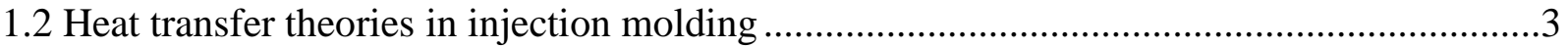

1.3 Feasible design space for conformal cooling and literature review .....................................

1.4 Temperature variance issue in conformal cooling ........................................................12

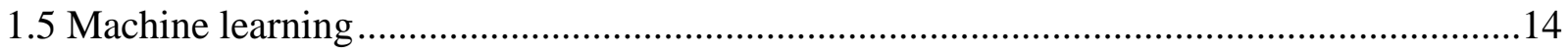

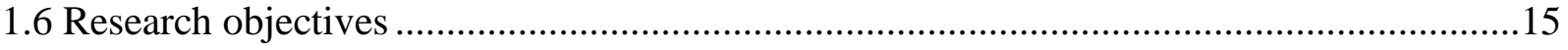

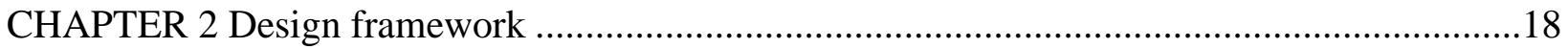

CHAPTER 3 Identification of design parameters and division of cooling surface .......................23

3.1 Identification of design parameters ................................................................................2

3.1.1 Spiral, zigzag, and porous cooling systems .............................................................24

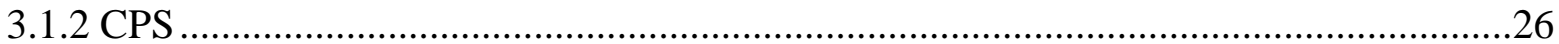

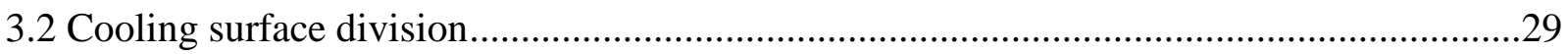

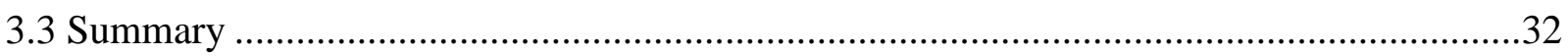

CHAPTER 4 Machine learning temperature prediction model.....................................................33

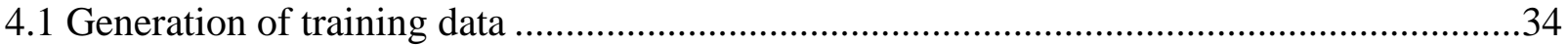

4.1.1 Algorithm design for the generation of zigzag cooling channels ..................................35

4.1.2 Algorithm design for the generation of spiral cooling channels ...................................38

4.1.3 Algorithm design for generation of porous and CPS cooling channels .........................42

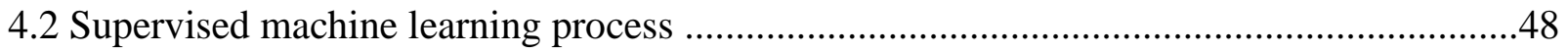

4.2.1 ANN training process .............................................................................................49

4.2.2 Evaluation of the surrogate temperature model.......................................................53

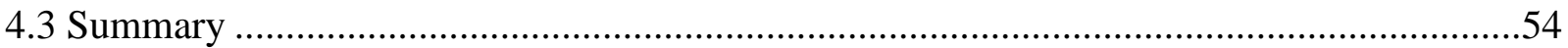

CHAPTER 5 Temperature variance minimization process ....................................................56

5.1 Optimization of spiral cooling channels .......................................................................56

5.2 Optimization of zigzag cooling channels ..........................................................................63 


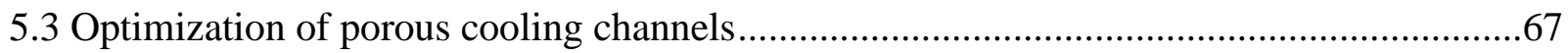

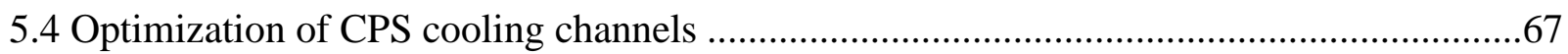

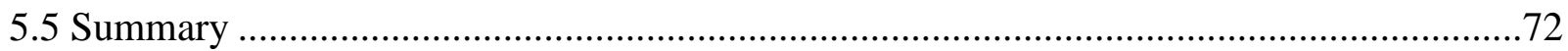

CHAPTER 6 Validation case studies and implementation of MLACCD software ......................73

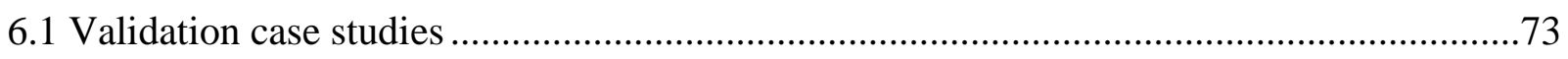

6.1.1 Freeform part surface and shoe sole …………..........................................................74

6.1.2 Swimming pedal ....................................................................................................

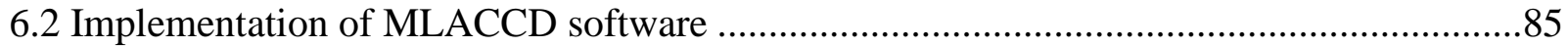

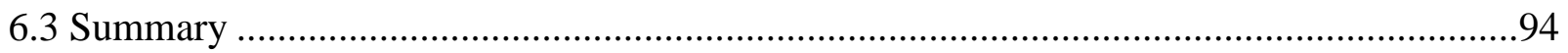

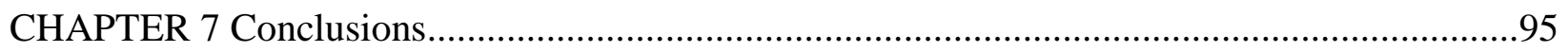

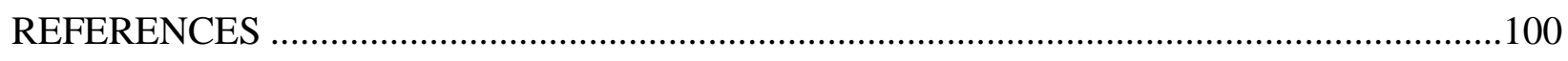

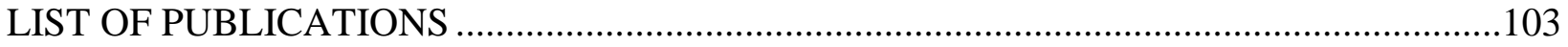




\section{LIST OF FIGURES}

Figure 1-1. The proportion of time spent on a typical injection molding cycle ........................ 1

Figure 1-2. A typical injection molding machine [6] ................................................... 2

Figure 1-3. Injection molding schematic: (a) a plunger; (b) a reciprocating rotating screw [1].... 2

Figure 1-4. Heat transfer in a conformal cooling cell .................................................. 4

Figure 1-5. The porous structure heat transfer model .................................................. 6

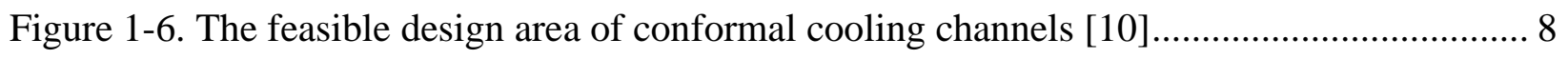

Figure 1-7. Temperature variance caused by the part thickness variation.............................. 13

Figure 2-1. Design procedure of a Voronoi conformal cooling circuit [15] ............................ 18

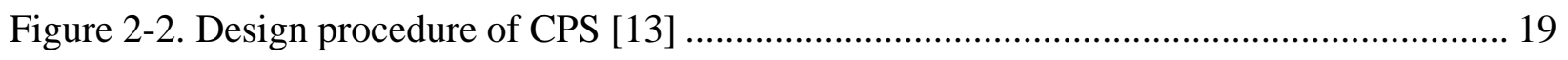

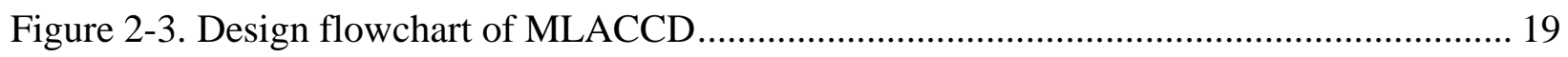

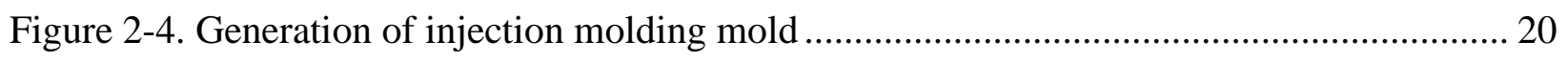

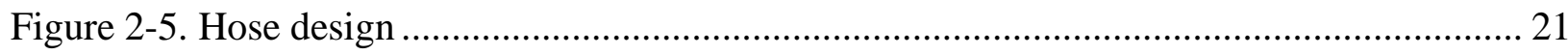

Figure 3-1 Design parameters for spiral, zigzag, and porous cooling channels ....................... 24

Figure 3-2. The coupling effect of changing the diameters of cooling channels ....................... 25

Figure 3-3. Required design parameters for a three-layer CPS ........................................ 26

Figure 3-4. The simulation configuration and an example temperature collection for trial

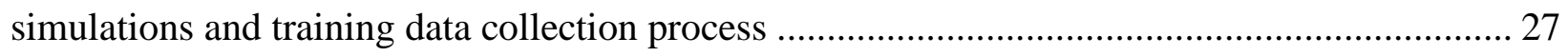

Figure 3-5. The cooling efficiency variation directions $C E V$ for each cooling channel design ... 30

Figure 3-6. General procedure of the cooling surface division .......................................... 31

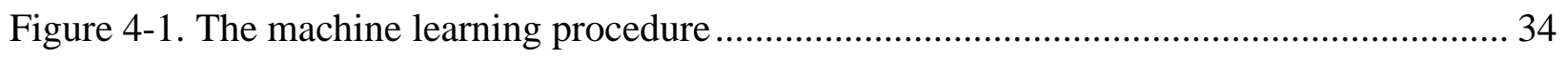

Figure 4-2. The zigzag simulation files generation algorithm ........................................ 35

Figure 4-3. The coordinates of the cooling channel control points and the block object edges ... 36 
Figure 4-4. Example simulation configuration and temperature distribution result for zigzag

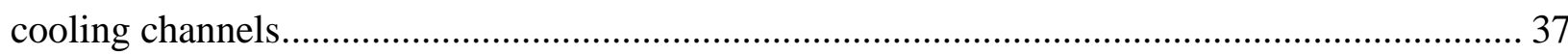

Figure 4-5. Simplified spiral BDM method ............................................................... 38

Figure 4-6. The part design and the relative part to channels positions ................................. 40

Figure 4-7. The spiral simulation files generation algorithm .......................................... 41

Figure 4-8. Example simulation configuration and temperature distribution result for spiral

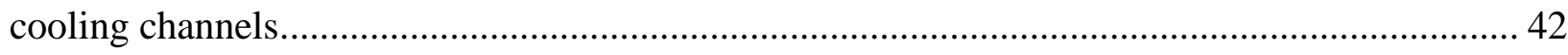

Figure 4-9. The CPS cooling channels generation method................................................. 43

Figure 4-10. The porous cooling channels generation method ............................................. 44

Figure 4-11. The CPS and porous structure simulation files generation algorithm.................. 46

Figure 4-12. Example simulation configuration and temperature distribution result for porous

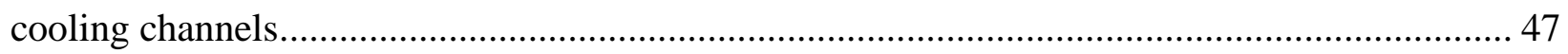

Figure 4-13. The ANN layout for different cooling channel topologies ................................ 50

Figure 4-14. Loss function of the machine learning process ........................................... 51

Figure 4-15. Training data for the supervised machine learning process ............................. 52

Figure 4-16. Part temperature prediction from the machine learning surrogate model.............. 53

Figure 5-1. The design procedure of machine learning aided spiral cooling channels............... 57

Figure 5-2. Division of spiral design areas from multiple local thickness maxima .................. 57

Figure 5-3. An example of a spiral cooling surface with gradient function and control lines...... 58

Figure 5-4. Generation of WOCP on a spiral cooling surface .......................................... 58

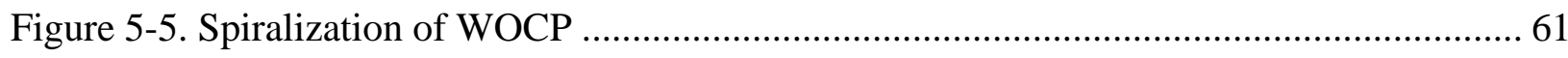

Figure 5-6. Generation of $l m$ - optimized control points of spiral cooling channels................. 61

Figure 5-7. Machine learning aided spiral cooling channel.............................................. 62 
Figure 5-8. The optimization procedure of zigzag cooling channels. 63

Figure 5-9. An example of a zigzag cooling surface with gradient function and control lines .... 64

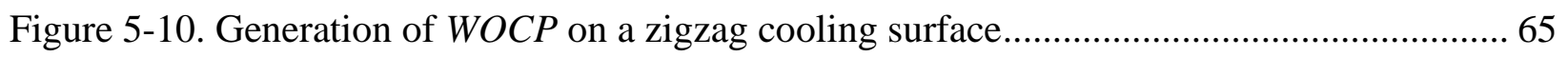

Figure 5-11. Generation of $l m$ - optimized control points of zigzag cooling channels ................ 66

Figure 5-12. Machine learning aided zigzag cooling channel ............................................. 66

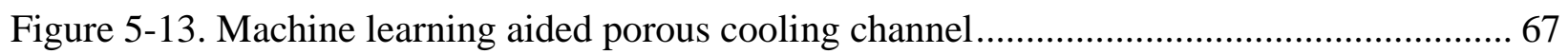

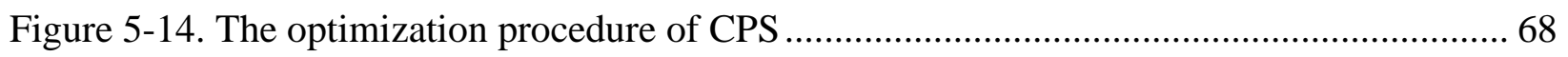

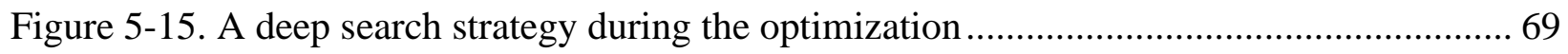

Figure 5-16. Design data and the optimized design data ................................................ 71

Figure 5-17. Machine learning aided CPS ........................................................................ 71

Figure 6-1. The part designs of case studies ........................................................................ 73

Figure 6-2. Comparison of CCCD and MLACCD channels ............................................ 75

Figure 6-3. The temperature distribution and the temperature variance of the part surface achieved by MLACCD channels and conventional conformal cooling channels ..................... 76

Figure 6-4. The coolant temperature distribution of the cooling channels for MLACCD channels

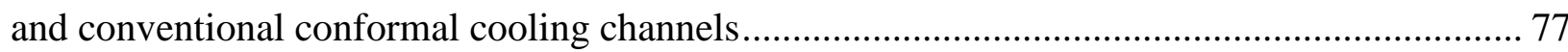

Figure 6-5. The part surface temperature distribution along the center of the part geometry for MLACCD channels and conventional conformal cooling channels..................................... 78

Figure 6-6. The coolant flow rate distribution of MLACCD channels................................... 79

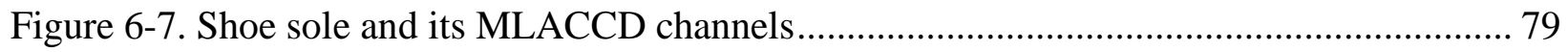

Figure 6-8. The temperature distribution and the temperature variance of the part surface achieved by MLACCD channels and conventional conformal cooling channels ..... 80 
Figure 6-9. The part surface temperature distribution along the center of the part geometry for MLACCD channels and conventional conformal cooling channels. 81

Figure 6-10. State-of-the-art conformal cooling channel topologies............................................. 81

Figure 6-11. Coolant pressure drop for MLACCD channels and conventional conformal cooling

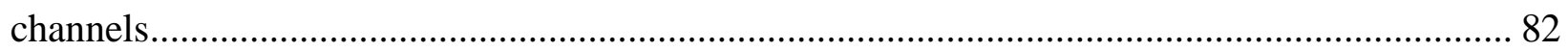

Figure 6-12. Machine learning aided CPS and conventional CPS ……………....................... 83

Figure 6-13. Resulting part temperature distribution for machine learning aided CPS and

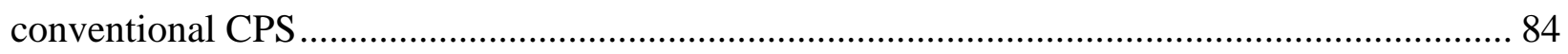

Figure 6-14. Resulting part temperature variance for machine learning aided CPS and

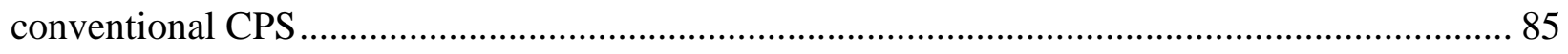

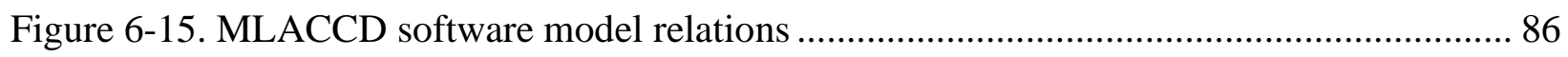

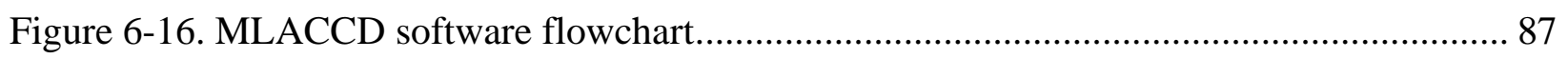

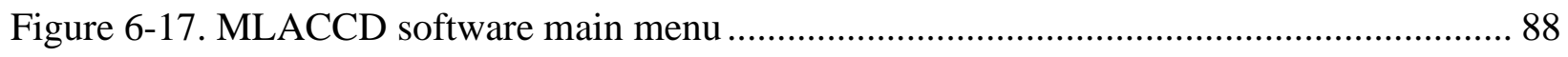

Figure 6-18. Design topology selection menu ........................................................................ 89

Figure 6-19. Temperature variance minimization (TVM) setting menu ....................................... 90

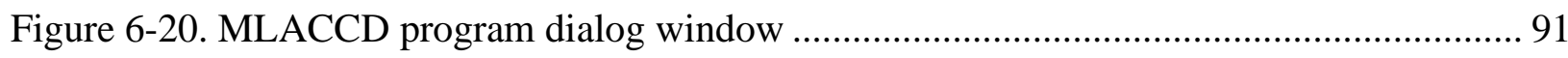

Figure 6-21. Example cooling surfaces …………………….............................................. 91

Figure 6-22. MLACCD CPS cooling channels for example cooling surfaces ............................ 92

Figure 6-23. Generation of cooling channels for the part with large geometrical angle variations

Figure 6-24. Generation of cooling channels for the part with small features 93 


\section{LIST OF TABLES}

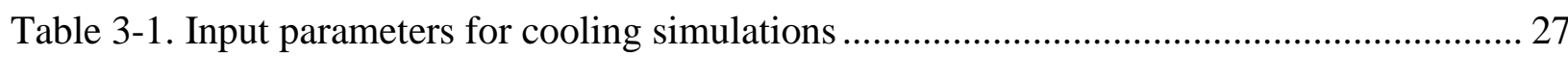

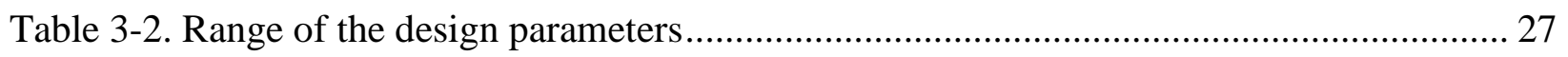

Table 3-3. Effectiveness evaluation results for cps design parameters ................................. 28

Table 4-1. Cooling simulations input parameters for training data collection ........................ 37

Table 4-2. Approximate computational cost of the simulation software and the machine learning

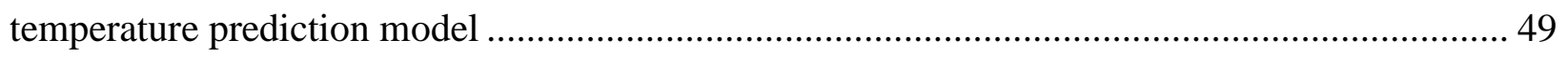

Table 4-3. The accuracy of the surrogate temperature prediction model ................................ 54

Table 5-1. The temperature variance minimization (TVM) strategies for each cooling topology

Table 5-2. Time efficiency of deep search algorithm and nested loop approach ..................... 70

Table 6-1. Parameters of injection molding cooling simulation for the case studies ................. 74

Table 6-2. A summary of simulation results for the first design case including: temperature

variance at critical locations, $\sigma_{\mathrm{T}}$, and the pressure drop .............................................. 75 


\begin{abstract}
The effectiveness of the cooling system in the injection molding processes significantly affects production efficiency and part quality. The current cooling system design is strictly limited by conventional manufacturing processes such as the casting and drilling process, which typically create straight or simple cooling channels. However, the maturing additive manufacturing (AM) technology allows the design and fabrication of complex conformal cooling channels. Typical advantages of conformal cooling are reduced cooling cycle time, smaller temperature variance, and better cooling quality. However, the existing conformal cooling designs do not support parts with non-uniform thickness values, which leads to high temperature variance. To improve the conformal cooling design in terms of the temperature variance, a machine learning aided design method is proposed to create cooling system which conforms not only to the part surface but also to the part thickness values. A surrogate part temperature prediction model is trained through a supervised machine learning process. Based on this model, optimization strategies are applied to different cooling topologies including zigzag, spiral, porous, and conformal porous structures (CPS) so that the resulting temperature variance of the part is minimized.
\end{abstract}




\section{RÉSUMÉ}

L'efficacité du système de refroidissement dans les processus de moulage par injection affecte considérablement l'efficacité de production et la qualité des pièces. La conception actuelle du système de refroidissement est strictement limitée par les processus de fabrication conventionnels tels que le processus de coulée et de forage, qui créent généralement des canaux de refroidissement droits ou simples. Cependant, la technologie de fabrication additive (AM) en cours de maturation permet la conception et la fabrication de canaux de refroidissement conformes et complexes. Les avantages typiques du refroidissement conforme consister à une réduction du temps de cycle de refroidissement, une plus petite variance de température et une meilleure qualité de refroidissement. Cependant, les conceptions de refroidissement conformes existantes ne prennent pas en charge les pièces dont les valeurs d'épaisseur ne sont pas uniformes, ce qui entraîne une variance de température élevée. Pour améliorer la conception de refroidissement conforme en termes de variance de température, une méthode de conception assistée par apprentissage automatique est proposée pour créer un système de refroidissement qui se conforme non seulement à la surface de la pièce mais également aux valeurs d'épaisseur de la pièce. Un modèle de prédiction de

température de pièce de substitution est formé par un processus d'apprentissage automatique supervisé. Sur la base de ce modèle, des stratégies d'optimization sont appliquées sur différentes topologies de refroidissement, y compris les structures poreuses en zigzag, en spirale, poreuses et conformes (CPS) de sorte que la variance de température résultante de la pièce soit minimisée. 


\section{ACKNOWLEDGMENTS}

First, I want to express my sincere gratitude to my supervisor, Yaoyao Fiona Zhao, for offering me this opportunity to work and contribute in the additive manufacturing industry, and patiently guiding my research in the past two years. I'm very grateful that she is always supportive when I try to propose my own research idea to solve the problems, and giving me much thoughtful and useful advice to help me improve my research. It is very a pleasure to work with her in this research field.

I also want to thank to the financial support from NSERC Strategic Network for Holistic Innovation in Additive Manufacturing (HI-AM) Grant No. NETGP 494158-16 and NSERC Discovery Accelerator Supplements program RGPAS-2018-522708, which makes my international study and research at McGill University possible.

Finally, I would like to thank to my parents, Hong Dang and Kunhua Gao, who have continuously given me financial and mental support. I also want to give my deepest love and gratefulness to my fiancée, Shiheng Liu, who has accompanied me throughout the study, brought the happiness to my life, and encourages me to bravely conquer any challenges that I encounter. 


\section{CONTRIBUTION OF AUTHUORS}

The final thesis reflects beneficial advice and comments provided by thesis supervisor Prof.

Yaoyao Fiona Zhao. In addition to this, all work presented below was conducted by the author. 


\section{CHAPTER 1 \\ Introduction}

\subsection{Background}

As a common industrial manufacturing process, the injection molding method fabricates parts by injecting molten material into the mold [1]. With the pre-designed mold and cooling systems, parts with complicated geometry can be produced within a short time [2]. The injection molding technology is widely applied in aerospace [3], automotive [4], and plastics industries [5]. A typical injection molding cycle includes six procedures: (1) mold close, (2) filling, (3) pack and hold, (4) cooling and recovery, (5) mold open, (6) ejection as shown in Figure 1-1. At the beginning of an

- Mold Close
Fill Time
- Pack and Hold
- Cooling and Recovery
Mold Open
- Ejection

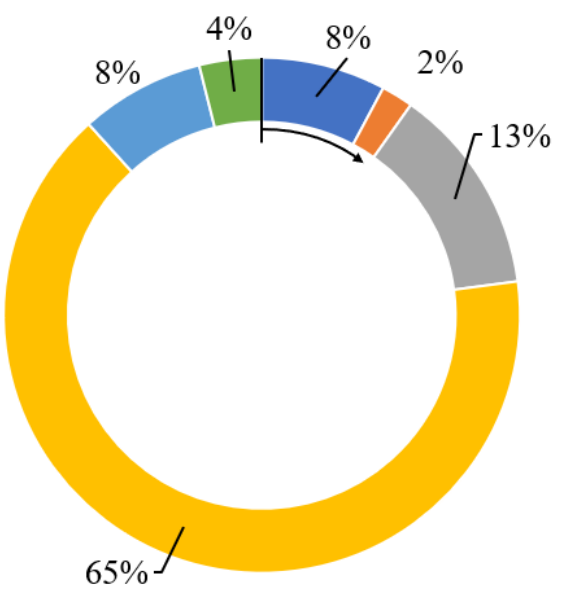

Figure 1-1. The proportion of time spent on a typical injection molding cycle

injection molding cycle, the mold is closed to generate a sealed cavity for the liquid of molten plastic. The liquid plastic is initially plastic powder and pellets, which are imported from the hopper container, and melted in the cylindrical injection area. These materials are transferred through the sprue into the mold by the rotating and reciprocating screw as shown in Figure 1-2 [6], where some injection molding machines also use a plunger for the transfer of the molten plastic as shown in Figure 1-3 [1]. Afterwards, the molten material is packed and held within the mold for 


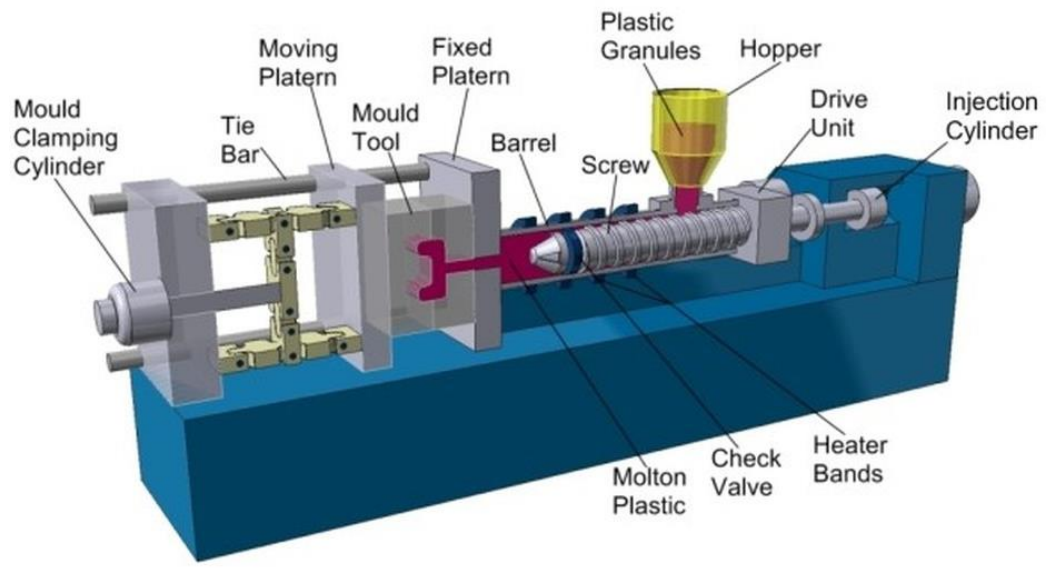

Figure 1-2. A typical injection molding machine [6]

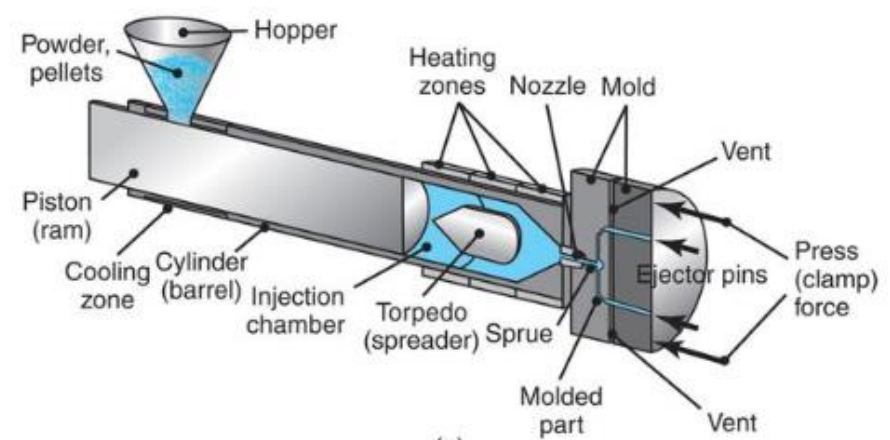

(a)

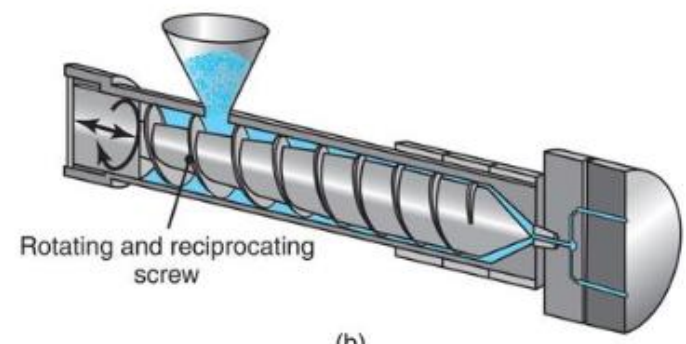

(b)

Figure 1-3. Injection molding schematic: (a) a plunger; (b) a reciprocating rotating screw [1]

4 to 6 seconds. The liquid plastic is then solidified by the cooling system of the mold. Finally, the mold is opened, and the complete product is ejected after the cooling process. During these procedures, the cooling and recovery takes $60 \%$ to $70 \%$ of the overall cycle time. In addition, it significantly affects the resulting quality and the dimensional accuracy of the product [7]. Thus, the cooling system design has a critical effect on the production cycle time and part quality. 
Nevertheless, the limitation of the conventional drilling and casting process imposes constraints in designing complex and effective cooling channels. Traditionally, the cooling system of the injection molding machine is either designed with straight cooling channels [8], or an array of cooling baffles [9]. These conventional cooling channels are not necessarily conformal to the part geometry, which results in a longer production cycle time and poor quality of the part [10]. However, the maturing Additive Manufacturing (AM) technology makes the fabrication of true conformal cooling channels possible with reasonable time and cost [11]. In this chapter, a review of theoretical works of conformal cooling heat transfer are first provided in Section 1.2. Feasibility of conformal cooling design and the conformal cooling literature review are given in Section 1.3. In Section 1.4, the problems and challenges for existing conformal cooling systems including the part temperature variance and the coolant pressure drop are discussed. To solve the temperature variance problem, a machine learning aided approach solution is proposed in Section 1.5. In Section 1.6, the objective of this research is discussed, and the organization of the thesis is provided.

\subsection{Heat transfer theories in injection molding}

The conformal cooling channel is defined as the cooling passageway which is conformal to the geometrical profile of the mold core, so that a rapid cooling efficiency and an uniform cooling performance of the cooling system are achieved [12]. The heat transfer theory and design guideline of conformal cooling channels are first proposed by Xu et al. [10]. Based on their model, the energy conservation of the mold during the cooling process can be molded by equation (1-1):

$$
E_{p}=E_{m}+E_{c}
$$

where $E_{p}$ is the heat transferred from half of the part to the mold, $E_{c}$ is the energy loss through the coolant, and $E_{m}$ is the energy accumulated in the mold. The schematic of this energy relation is 


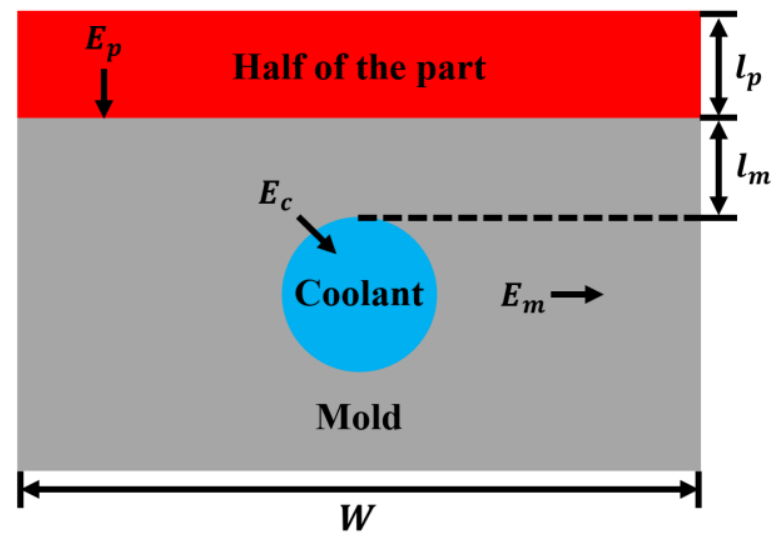

Figure 1-4. Heat transfer in a conformal cooling cell

provided in Figure 1-4. According to the heat transfer theories, equation (1-2) to equation (1-5) are derived:

$$
\begin{gathered}
E_{p}=\rho_{p} c_{p} l_{p} W\left(T_{\text {melt }}-T_{\text {eject }}\right) \mathrm{dt} / t_{\text {cycle }} \\
E_{m}=\rho_{m} c_{m} l_{m} W\left(T_{m}(\mathrm{t}+\mathrm{dt})-T_{m}(\mathrm{t})\right) \\
E_{c}=K_{m} W\left(T_{m}(\mathrm{t})-T_{a}(\mathrm{t})\right) \mathrm{dt} / l_{m} \\
E_{c}=\frac{1}{2} h \pi D\left(T_{a}(\mathrm{t})-T_{c}\right) \mathrm{dt}
\end{gathered}
$$

where equation (1-2) is derived based on the average energy loss in a time dt over the total cycle time $t_{c y c l e}$ during a cooling cycle for a part (density $\rho_{p}$, specific heat capacity $c_{p}$, half of the thickness $l_{p}$ ) cooled from its melt temperature $T_{\text {melt }}$ to the ejection temperature $T_{\text {eject }}$. Equation (1-3) represents the heat accumulated within the mold of a cooling cell (density $\rho_{m}$, specific heat capacity $c_{m}$, pitch width $W$, pitch to mold surface distance $l_{m}$ ) due to the mold temperature raises from $T_{m}(\mathrm{t})$ to $T_{m}(\mathrm{t}+\mathrm{dt})$. Equations (1-4) and (1-5) are constructed based on the heat flux at the mold-coolant interface, where $K_{m}$ is the heat conductivity of the mold material, $h$ is the heat transfer coefficient at the mold-coolant interface, $D$ is the diameter of the cooling channel, $T_{a}(\mathrm{t})$ 
is the cycle averaged temperature at the mold-coolant interface. To solve $T_{a}(\mathrm{t})$, equation (1-4) and equation (1-5) are combined in equation (1-6):

$$
\frac{K_{m} W\left(T_{m}(\mathrm{t})-T_{a}(\mathrm{t})\right) \mathrm{dt}}{l_{m}}=\frac{1}{2} h \pi D\left(T_{a}(\mathrm{t})-T_{c}\right) \mathrm{dt}
$$

where the resulted $T_{a}(\mathrm{t})$ is given in equation (1-7):

$$
T_{a}(\mathrm{t})=\frac{K_{m} W T_{m}(\mathrm{t})+\frac{1}{2} h \pi D l_{m} T_{c}}{K_{m} W+\frac{1}{2} h \pi D l_{m}}
$$

submitting equation (1-7) to equation (1-4), equation (1-8) is obtained using the relation from equation (1-1) and the heat transfer calculations in equations (1-2) to (1-4).

$$
\frac{\rho_{m} c_{m} l_{m} W\left(T_{m}(\mathrm{t}+\mathrm{dt})-T_{m}(\mathrm{t})\right)}{\mathrm{dt}}+\frac{h \pi D K_{m}}{2 K_{m} W+h \pi D l_{m}}\left(T_{m}(t)-T_{c}\right)=\frac{\rho_{p} c_{p} l_{p}\left(T_{\text {melt }}-T_{\text {eject }}\right)}{t_{c y c l e}}
$$

By applying $\lim _{\mathrm{dt} \rightarrow 0}$ to equation (1-8), a first order differential equation is obtained:

$$
\rho_{m} c_{m} l_{m} W \frac{\mathrm{d} T_{m}(t)}{\mathrm{dt}}+\frac{h \pi D K_{m}}{2 K_{m} W+h \pi D l_{m}}\left(T_{m}(t)-T_{c}\right)=\frac{\rho_{p} c_{p} l_{p}\left(T_{m e l t}-T_{e j e c t}\right)}{t_{c y c l e}}
$$

where the solution of equation (1-9) is expressed in equation (1-10):

$$
T_{m}(t)=\overline{T_{m}}+\left(T_{m 0}-\overline{T_{m}}\right) e^{-t / \tau}
$$

where $\overline{T_{m}}$ is the cycle averaged mold temperature as shown in equation $(1-11), \tau$ is the time constant as shown in equation (1-12), and $T_{m 0}$ represents the initial mold temperature.

$$
\begin{gathered}
\overline{T_{m}}=T_{c}+\frac{\rho_{p} c_{p} l_{p}\left(2 K_{m} W+h \pi D l_{m}\right)\left(T_{m e l t}-T_{e j e c t}\right)}{h \pi D K_{m} t_{c y c l e}} \\
\tau=\frac{\rho_{m} c_{m} l_{m}\left(h \pi D l_{m}-2 K_{m} W\right)}{h \pi D K_{m}}
\end{gathered}
$$


Based on equation (1-10), the mold temperature of typical conformal cooling channels designed from offset cooling surface can be modeled.

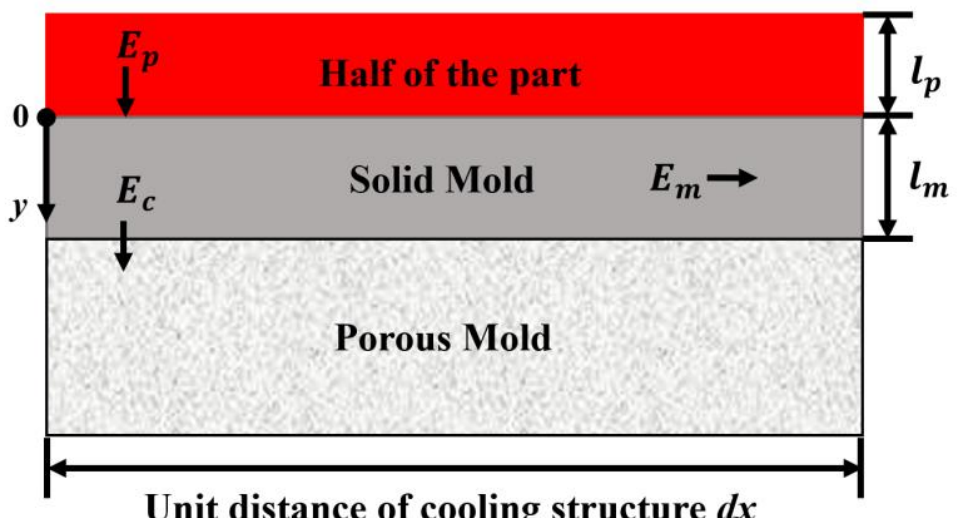

Figure 1-5. The porous structure heat transfer model

To improve the cooling efficiency and reduce the pressure drop of the mold, the porous cooling systems are another type of the cooling system that has recently been developed and studied. Thus, adaptations of the heat transfer model from $\mathrm{Xu}$ et al. [10] also exist for the mold temperature calculation of general porous conformal cooling topologies [13]. Instead of modeling the cooling structures with a circular cross section as shown in Figure 1-4, a rectangular chunk of porous cooling area beneath the mold material is considered as shown in Figure 1-5. Hence, the $E_{c}$ and $E_{m}$ in equation (1-1) are re-calculated in equation (1-13) and equation (1-14), where $\rho_{m}$ is the density of the solid mold material, $c_{m}$ is the specific heat capacity of the solid mold, $T_{\text {mold }}(t, y)$ represents the mold temperature function at time $t$, position $y, K_{m}$ is the heat conductivity of the mold material, $h$ is the effective heat convection coefficient for the solid and porous mold interface, and $T_{c}$ is the coolant temperature.

$$
\begin{gathered}
E_{m}=\rho_{m} c_{m} d x d z \int_{0}^{l_{m}}\left(T_{\text {mold }}(t+d t, y)-T_{\text {mold }}(t, y)\right) d y \\
E_{c}=\frac{h d x d z\left(K_{m} T_{m s u r f}(t)-K_{m} T_{c}\right)}{K_{m}+l_{m} h} d t
\end{gathered}
$$




$$
T_{m s u r f}(t)=\overline{T_{m s u r f}}+\left(T_{0}-\overline{T_{m s u r f}}\right) e^{-t / \tau}
$$

The modified average mold surface temperature $\overline{T_{m s u r f}}$ and the time constant $\tau$ for equation (1$15)$ is then derived in equation (1-16) and equation (1-17):

$$
\begin{gathered}
\overline{T_{\text {msurf }}=}\left(T_{c}+\frac{K_{m}+l_{m} h \rho_{p} c_{p} l_{p}\left(T_{\text {melt }}-T_{\text {eject }}\right)}{h K_{m} t_{c y c l e}}\right) \\
\tau=\frac{\rho_{m} c_{m} l_{m}\left(l_{m} h+2 K_{m}\right)}{2 h K_{m}}
\end{gathered}
$$

where $T_{\text {msurf }}(t)$ is the mold surface temperature at time $t, \overline{T_{\text {msurf }}}$ is the average mold surface temperature for an injection molding cycle, $\tau$ is a time constant, $\rho_{m}$ is the density of the solid mold material, $c_{m}$ is the specific heat capacity of the solid mold, $K_{m}$ is the heat conductivity of the mold material, $h$ is the effective heat convection coefficient for the solid and porous mold interface, $T_{c}$ is the coolant temperature, $T_{0}$ is the initial temperature of the mold, $\rho_{p}$ and $c_{p}$ are the density and specific heat capacity of the part.

To precisely predict the local part temperature however, the existing theoretical models are not sufficient. First, the resulting part surface temperature cannot be derived from $T_{m}(t)$ in equation (1-10) or $T_{m s u r f}(t)$ in equation (1-15), since they mainly reflect the thermal status of the mold during an injection molding cycle. Thus, it is impossible to utilize these theoretical models for a fast and accurate prediction of the local part temperature based on a given set of cooling channel design parameters. In addition, the assumption is too general for the precise prediction of local part temperature during the derivation process of porous conformal cooling theory. According to the derivation process proposed by Tang et al. [13], this model approximates the porous cooling channels to a cooling area with uniform heat flux $\dot{Q}$, which is clearly insufficient in supporting the design of CPS composed of cooling cells with different cell sizes that can have variety of heat flux 
values across the mold surface. Another shortcoming of the porous model is that it does not link the design parameters of the cooling channels to its resulting thermal effects on the mold.

\subsection{Feasible design space for conformal cooling and literature review}

For the feasible design area, $\mathrm{Xu}$ et al. [10] proposed a model relating the coolant pressure drop, uniformity of the cooling performance, geometric constraint of the mold, and the manufacturing limitations as shown in Figure 1-6 [10]. Note that this feasible design area will differ if the design of the part, mold, or conformal cooling channel design varies. Typically, a smaller diameter of the cooling channels results in an increase of the cooling efficiency but a reduction in terms of the manufacturability and mechanical properties (i.e. strength, damage tolerance) of the mold. Among different conformal cooling research and engineering analysis [14-16], the minimum diameter of the cooling channels is $6 \mathrm{~mm}$ balancing the manufacturability and the cooling efficiency of the cooling system. In this research, the minimum diameter of $6 \mathrm{~mm}$ is selected to achieve a high cooling performance of the cooling channels without introducing significant industrial fabrication difficulties.

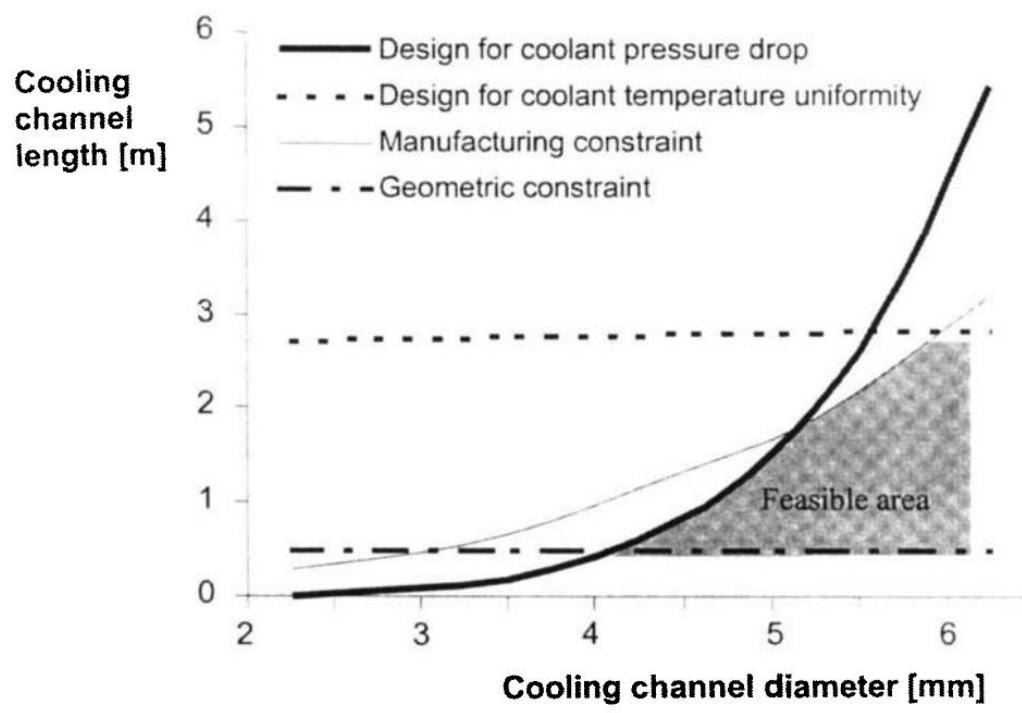

Figure 1-6. The feasible design area of conformal cooling channels [10] 
Although the heat transfer theory and design guideline of the conformal cooling channels have been established, the design of conformal cooling channel has only been investigated to very limited extent. Khan et al. [17] provided a cycle time reduction method by combining zigzag and parallel conformal cooling channel topologies to appropriate cooling surfaces. In their work, analytical comparison is made among different cooling topologies in terms of the cooling time, temperature variance, and the part warpage, where a suitable topological design of the conformal cooling channels can be provided based on a given design of the part. An automatic design of conformal cooling channels is studied by Wang et al. [15] by creating the centroidal Voronoi diagram (CVD) on the conformal surface. A geometric algorithm is developed in their work to automatically generate a Voronoi cooling surface which is conformal to the part in a short time. With their design method, the resulting volumetric shrinkage and the cooling time of the part is reduced. The CVD design is then compared with the spiral design method of conformal cooling channels proposed by Wang et al. [14], where the spiral design of conformal cooling channels is proven to have shorter cooling time and lower part temperature variance. The comparison results also indicate that the spiral cooling channels have advantages over Voronoi cooling channels in terms of fabrication cost and the uniformity of coolant temperature and Reynold number.

However, these conformal cooling channels are designed on cooling surfaces extracted and offset from the part surface geometry. Since the design of these cooling systems is limited to these 2D cooling surfaces, a lot of mold design space is not utilized to improve the cooling performance of the mold. Thus, the porous conformal cooling systems are developed to further reduce the cooling time and part temperature variations. These type of the cooling systems also have the advantages in terms of its small coolant pressure drop [18], mass reduction [19], and rapid cooling [20]. Specifically, Au et al. [21] proposed a multi-connected porous coolant passageway design 
with a finite number of cubical cooling cells. The fluid dynamics simulation results indicate that a more uniform cooling performance and a smaller number of part defects are achieved. Brooks et al. [22] designed a porous conformal cooling mold with self-supporting lattice structures. The resulting convective heat transfer of the mold is improved by its innovative cooling system design. Among different porous systems, the conformal porous structure (CPS) is an innovative porous structure proposed by Tang et al. [13], which is defined as a unique type of porous set of cooling channels that has the cooling cells conformal to the part surface. Since uniformity of the distance between the cooling cells and the mold surface is improved, a more uniform cooling performance, smaller temperature variance of the part, and reduced coolant pressure drop can be achieved. However, if the part to be molded has a lot of thickness variations, the large temperature variance along the part surface will be a severe issue for all the existing conformal cooling topologies.

A number of publications have reported on the optimization of design parameters of conformal cooling channels. A feature deposition method is provided by $\mathrm{Li}$ et al. [23] to recognize and separate the part geometry into different cooling areas. By designing the appropriate cooling channels into each cooling region, the resulting part surface temperature variance is reduced. Park et al. [24] provided the optimized design of baffles in their cooling system. The pitch-to-pitch distances of the baffles are designed with an optimized value, and the resulting cooling time is decreased. Jahan et al. [25] optimized the design of porous conformal cooling channel topology based on the mechanical behavior and thermal properties of 3D printed stainless steel porous conformal cooling molds with different mold porosities and structures. Two design cases of plastic parts with discrete thickness values ranging from $1 \mathrm{~mm}$ to $6 \mathrm{~mm}$ are also studied by Jahan et al. [26] using the design of experiment method (DOE). The optimized design of helix conformal cooling channels is able to minimize cooling time for plastic parts with a cylindrical and conical 
shape. To optimize the cellular cooling structures, Wu et al. [19] provided a multiscale lattice topology optimization method. In their research, an AM manufacturable mechanical stable mold is developed with rapid cooling capability. Although a $30 \%$ mass reduction of the mold is achieved with the same cooling performance, the optimized mold is not able to improve the part manufacturability and the quality due to temperature variance during the cooling process. In addition, Park et al. [16] has proposed an optimization method of conformal cooling channels based on the simulation results of the temperature distribution after the filling stage. It is found that the resulting cooling time is reduced by applying proper types of conformal cooling channels into different cooling regions of the part surface. The effect of mold porosity on its density, specific heat, and thermal conductivity is studied by Jahan et al. [27]. In their work, the porosity of the mold is optimized with respect to the cooling time for discrete thickness values of the part. Nevertheless, the optimization research related to part warpage minimization mainly focused on optimizing the parameters of the injection molding process or the design of part geometries. For instance, the optimization method of the injection molding gate location is proposed by Pandelidis et al. [28]. Moreover, Lee at al. [29] provided the optimization of part thickness design for warpage minimization. Nevertheless, there is only very limited research on the minimization of part surface temperature variance by optimizing the design parameters of cooling channels. There is also lack of research on the design and optimization of conformal cooling channels for the part with continuous thickness variations. In addition, the current cooling system design method for different part thickness cannot be easily adopted for large part thickness variation.

To properly handle the part surface temperature variance problem, the relation between the design parameters of the cooling channels, the part thickness values, and the resulting part surface temperature needs to be derived. Dimla et al. [30] proposed an optimization method of an injection 
molded part. To reduce the cycle time, the optimal part design, gate locations, and the cooling channels design are determined based on the FEA and thermal analysis result. However, there is still lack of detailed discussion and solution on the temperature variance caused by the part thickness variations in their research. To obtain an optimized design of cooling channels in injection molds, a design of experiments (DOEs) technique is applied by Jahan et al. [31] to investigate the design parameter effects of cooling channels on its cooling performance. Nevertheless, the proposed model in their work does not exhibit self-learning and improvement abilities if the design parameters change their limits. In addition, the proposed model and DOEs method is not suitable to be applied for more complicated conformal cooling channel design such as CPS. A part warpage optimization based on DOEs and Glowworm Swarm Optimization (GSO) method is proposed by Hazwan et al. [32]. In their study, the process parameters of the injection molding are optimized, and the resulting part warpage is reduced by $39.1 \%$. A multi-objective optimization of injection molding process parameters is also proposed by Kitayama et al. [33] to improve the cooling performance and reduce the part warpage. Still, due to the fact that the majority of the optimization research is focused on optimizing the injection molding process parameters, limited discussion is found regarding to the optimization for the conformal cooling channel design instead. These challenges call for the investigation of applying innovative temperature prediction techniques to aid the design and optimization of conformal cooling channels for the minimization of the temperature variance.

\subsection{Temperature variance issue in conformal cooling}

The temperature variance is one of the existing problems for the conformal cooling design research. It is typically caused by the non-uniformity of the part thickness distribution as shown in Figure 1-7. Specifically, part regions with different heat inertia are cooled by the channels distributed 


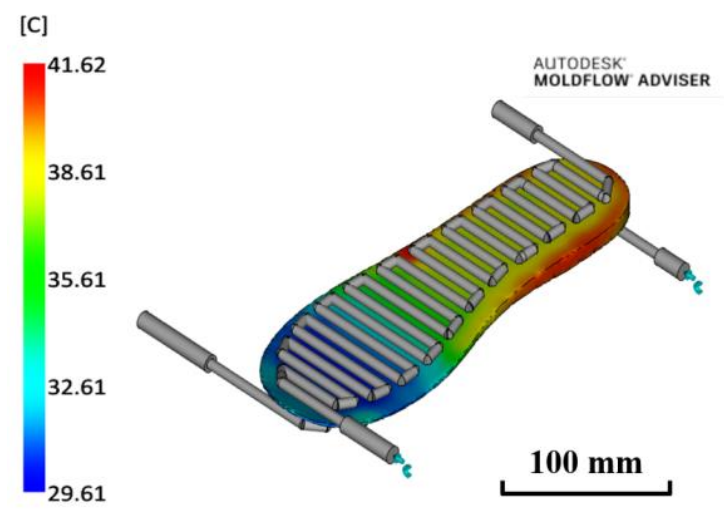

Figure 1-7. Temperature variance caused by the part thickness variation

above the part surface for a fixed distance with the same flow rate. The resulting non-uniform temperature along the part surface leads to thermal residual stress, which typically exists in the part regions that have large thickness variations. As indicated by Jacques et al. [34], the temperature variance of an injection molded part will cause the warpage problem and hence a poor part quality. In addition, it is stated by Shayfull et al. [35] that the warpage issue is even more likely to be caused by the temperature variance for the injection molded part with smaller thickness values. The existing literatures mainly focus on solving this issue by alternating the original design of the part [36] and optimizing the injection molding process parameters [37, 38]. Nevertheless, limited optimization efforts have been found on handling this issue with a properly designed cooling system. Since the pressure drop of the injection molding cooling systems is already significantly reduced by different novel designs such as multi-connected porous structures and CPS $[13,21]$, the temperature variance is the major remaining problems for current conformal cooling research. To solve the temperature variance problem of a part with non-uniform thicknesses, a prediction of the local part surface temperature is required based on a given set of cooling system design parameters. However, precisely predicting the local part surface temperature remains unsolved. This is due to the complexity of the heat transfer process in the 
injection molding, where running a large amount of cooling simulations to predict the part surface temperature during the optimization process of the conformal cooling channels is unrealistic. Therefore, an accurate yet fast prediction tool of the local part surface temperature is required to further study and solve the temperature variance issue.

\subsection{Machine learning}

The main challenge of handling the temperature variance problem is the fast and accurate prediction of the local part temperature based on the given cooling channel design parameters. Due to the complexity of heat transfer during an injection molding cycle, it is difficult to link the design parameters of different conformal cooling topologies to the resulting part surface temperature precisely. However, this type of problem is a well-suited candidate for machine learning. Machine learning, as a subset of artificial intelligence, has attracted a lot of research interests in the recent years. It is defined as a set of methods which are able to automatically detect data patterns to predict future data or make decisions based on uncovered patterns of data [39]. With a sufficient volume of data, the machine learning will be an ideal temperature prediction tool to detect underlying trends and patterns of the relations between the resulting part temperature and design parameters [40].

Recently, different machine learning strategies have been applied to improve the manufacturing industry. An agent-based (holonic) AI systems is proposed by Monostori [41] to handle the complexity, changes and disturbances in production systems. Instead of using dispatching rules, Priore et al [42] applied inductive learning, backpropagation neural networks, and case-based reasoning (CBR) to schedule the flexible manufacturing systems. A significant improvement over the conventional dispatching rules is obtained by their machine learning approach. Chi et al [43] developed an adaptive Automated Intelligent Manufacturing System 
(AIMS) to aid the pharmaceutical development and manufacturing process. In their study, the AIMS is proofed to be both explanatory and predictive. More machine learning aided approach in manufacturing processes is reviewed by Priore et al [44]. For the injection molding industry, Park et al. [45] proposed a real-time intelligent control of the injection molding process parameters. Based on their method, a smart and automatic injection molding process is achieved by training the algorithm with the machine learning method. Nevertheless, the machine learning approach in their work is focused more on the process parameters aspects, and there is not much discussion on the improvement of the cooling channel designs. Shi et al. [46] shows that the injection molding process can be optimized with an artificial neural network (ANN) algorithm to reduce the part warpage. In their research, an ANN surrogate model is built to approximate the relations between the design variables of the injection molding process and part quality index. However, there is limited research effort found so far on the machine learning aided approach to design the conformal cooling channels. Thus, it is worthwhile to investigate the machine learning assisted design and optimization of the cooling system by training a surrogate model to predict the resulting part surface temperature.

\subsection{Research objectives}

The goal of this research is to study and solve temperature variance issue caused by the nonuniform thickness distribution of the part in conformal cooling research, where several challenges exist. As stated in Section 1.5, machine learning is selected as the temperature prediction tool in this research to achieve an efficient and precise part temperature prediction. To train the machine learning model, however, the training data need to be properly selected for different cooling channel topologies (i.e. effective design parameters need to be identified, sufficient amount of training data need to be gathered, etc.). Once the temperature prediction is achieved by the 
machine learning method, optimization algorithms need to be designed for different cooling channel topologies to finally generate the cooling channels with machine learning aided conformal cooling design (MLACCD). To address these challenges, there are in total four research objectives in this thesis:

1) To identify the design parameters of different conformal cooling topologies including zigzag, spiral, porous, and CPS. These topologies are chosen due to their validated effective cooling performance in the reported literatures $[13,14,16,21]$. The selected design parameters should be both essential in geometrical construction of the cooling channels, and effective in influencing the resulting local cooling performance.

2) To build sufficient amount of training data for the supervised machine learning process. The Moldflow Advisor ${ }^{\circledR}$ simulation software is selected in this work to generate the training data, where the accuracy of this simulation software is validated via previous research on different cooling systems [14-16, 24]. Since large amount of training data is required to train the machine learning algorithm, specific programs need to be developed to automatically generate simulation files to gather the required training data.

3) To train the surrogate temperature prediction through the supervised machine learning process. The resulting temperature prediction model should be able to efficiently and precisely predict a local part surface temperature based on the given part thickness values and the conformal cooling design parameters.

4) To develop optimization strategies that minimize the temperature variance of different cooling channel topologies. With the surrogate temperature prediction tool, the optimizations need to be performed so that the MLACCD can be finally achieved. Due to the large design variations among different cooling channels topologies in terms of their geometrical 
distribution of the cooling channels, different optimization method is developed for each topological design of the conformal cooling system. Each optimization strategy should be time efficient, and able to effectively minimize temperature variance along the part surface by updating the wireframe design of conformal cooling channels.

With these objectives, the rest of the thesis is organized as follows. The design framework of the MLACCD is given in Chapter 2. In Chapter 3, the design parameter identifications of zigzag, spiral, porous, and CPS cooling topologies are provided, and a cooling surface division method is proposed. To discuss the machine learning strategy of this work, the training data generation and collection, supervised machine learning process, and the performance of the surrogate temperature prediction model is discussed in Chapter 4. In Chapter 5, the optimization strategies for zigzag, spiral, porous, and CPS cooling channels are delivered. This is followed by Chapter 6, where the proposed method is compared with conventional conformal cooling designs on different case studies including a freeform part surface, shoe sole, and a swimming pedal. In Chapter 7, a comprehensive summary of this research is made, and the potential future research directions are concluded. 


\section{CHAPTER 2}

\section{Design framework}

In this chapter, the overall design procedure of the machine learning aided conformal cooling design (MLACCD) is provided. Traditionally, the conformal cooling channels are created on a cooling surface that is offset from the part surface geometry as shown in Figure 2-1 [15], or within a design volume with uniform distributions of cooling structures as shown in Figure 2-2 [13]. Typically, the existing conformal cooling designs are constrained by the conventional conformal cooling concept, which requires the cooling channels to have a uniform cooling efficiency on the part surface (i.e. uniform pitch to mold surface distance $l_{m}$, pitch width $W$, etc.). Thus, the traditional conformal cooling design procedure is not suitable for the proposed MLACCD method due to two limitations: (1) the design parameters of MLACCD channels are not necessarily uniform (i.e. non-uniform pitch to mold surface distance $l_{m}$ ) since they are alternated and optimized from their original values based on the thickness distribution of the part; (2) the design concept of MLACCD involves new design procedures such as the training of the supervised machine learning surrogate part temperature model and the optimization of the conformal cooling channel design parameters using the machine learning part temperature prediction, which is totally different from the traditional conformal cooling design.

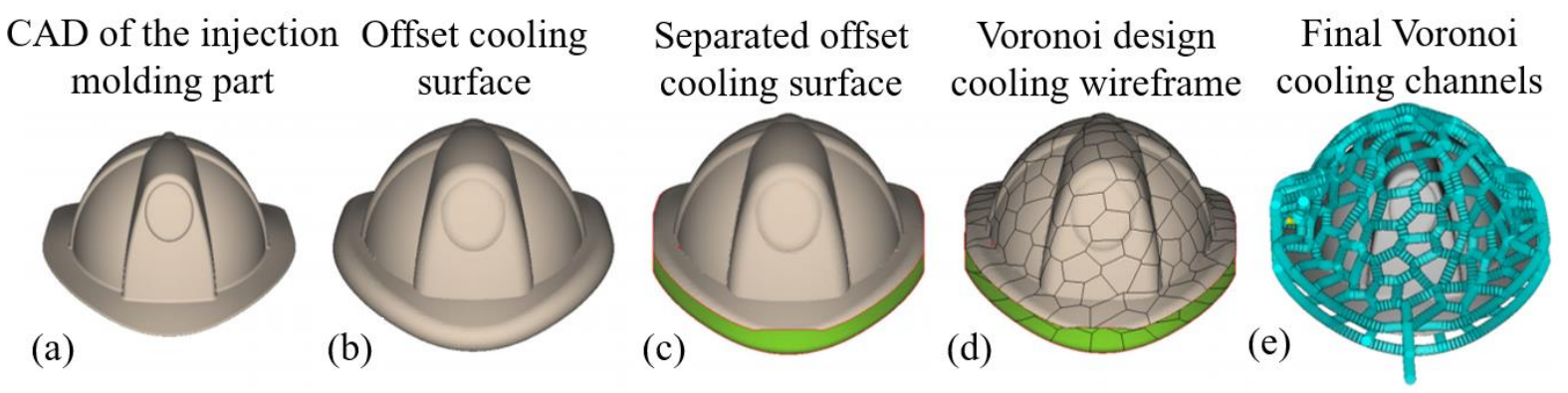

Figure 2-1. Design procedure of a Voronoi conformal cooling circuit [15] 


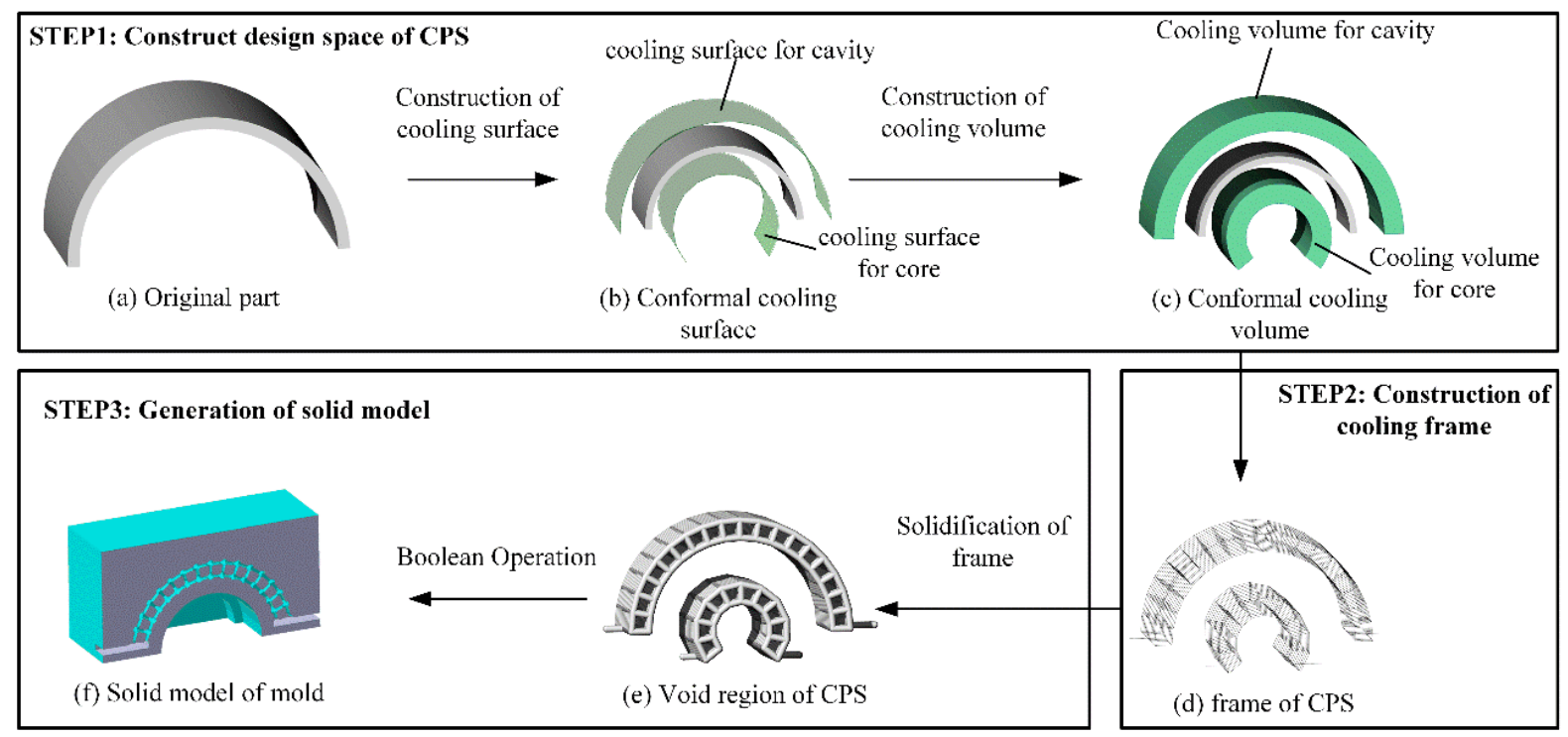

Figure 2-2. Design procedure of CPS [13]

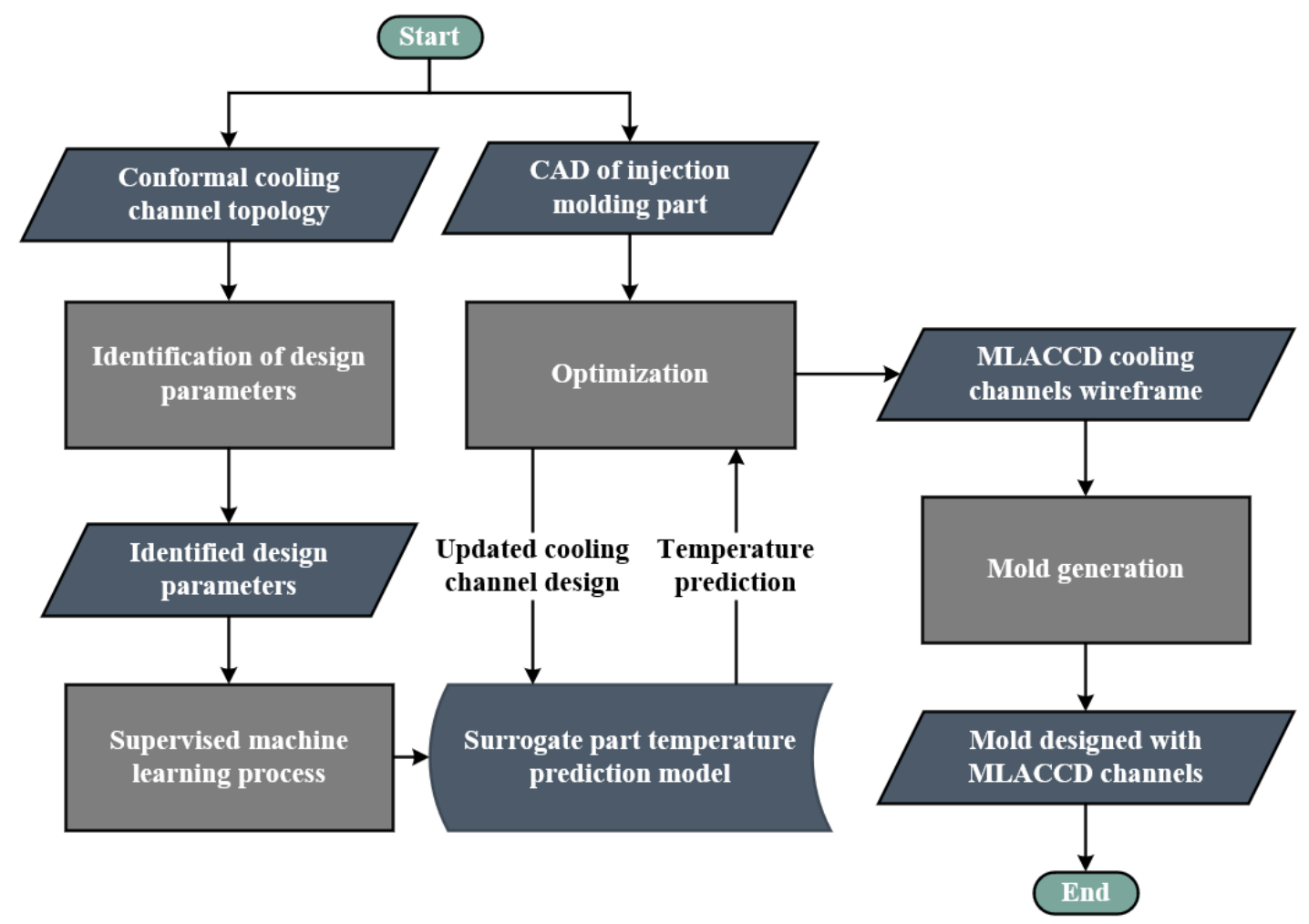

Figure 2-3. Design flowchart of MLACCD

The design flowchart of the MLACCD is provided in Figure 2-3, which consists of four steps:

(1) identification of design parameters; (2) supervised machine learning process; (3) optimization; 
(4) mold generation. A more detailed explanation of step (1) to step (3) is given in Chapter 3 to Chapter 5, so only a brief overview of those steps is given here. For a given conformal cooling topology, the design parameters are first identified. Based on these design parameters, cooling simulations are performed on Moldflow Advisor ${ }^{\circledR}$ to gather the resulting part temperatures for the supervised machine learning process. During the supervised machine learning process, the training data are fed into a two-layer ANN to train the surrogate part temperature prediction model. Note that the quality of the training process is tested by the loss function, and the accuracy of the model is evaluated by the test data randomly selected from the training data. Based on the surrogate temperature prediction model and the CAD design of the injection molding part, the optimization of the design parameters is performed and the wireframe of conformal cooling channels is generated. This optimized wireframe is used to construct the solid MLACCD channels so that a MLACCD injection molding mold can be built by the procedure shown in Figure 2-4. Typically,

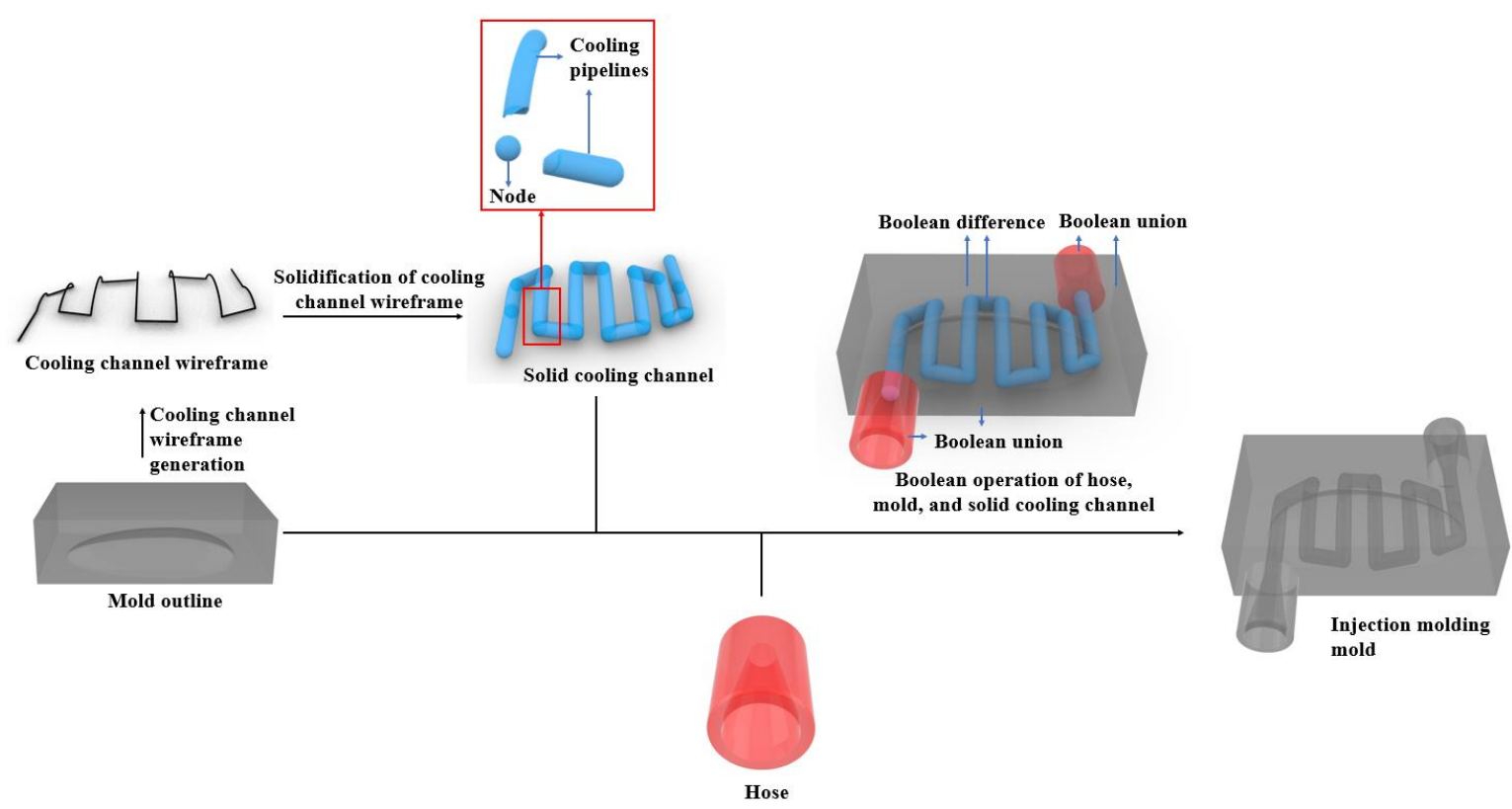

Figure 2-4. Generation of injection molding mold 
the generation of the solid conformal cooling channels is composed of the designs of nodes and cooling pipelines. According to Tang et al. [13], the relation between the diameters of nodes and the connected cooling channels is modeled in equation (2-1):

$$
D_{\text {node }}=C \frac{D_{\text {channel }}}{\sin \frac{\theta}{2}}
$$

where $D_{\text {node }}$ is the diameter of the node, $D_{\text {channel }}$ is the diameter of the channel, $\theta$ represents the minimum angle between the cooling channels connected at the node, $C$ is a constant greater than one, which avoids intersections between two connected cooling channels. In addition, the hoses are usually required to form a proper connection between the coolant pipes and the mold. The hose design of the mold is provided in Figure 2-5. The inner diameter of the hose usually exhibits

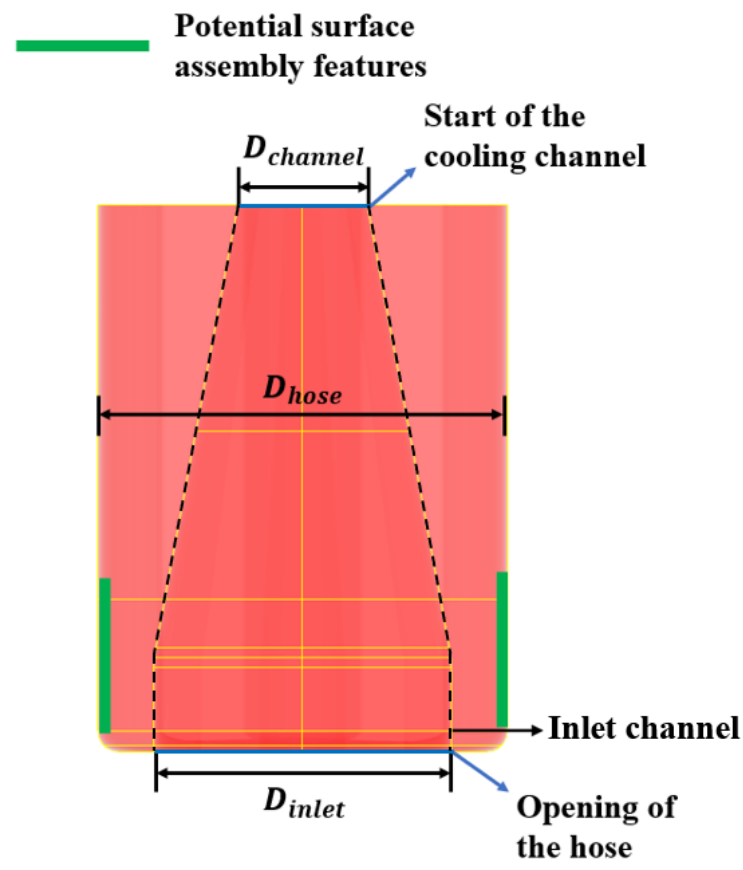

Figure 2-5. Hose design

a steady decrease from the opening of the hose $D_{\text {inlet }}$ to the start point of the cooling channels $D_{\text {channel }}$ to provide a smaller pressure drop of the coolant at inlet locations. The outer surface 
design of the hose is typically determined by the dimensions and the assembly features of the coolant inlet pipes (i.e. in Figure 2-5, the $D_{\text {hose }}$ depends on the inner diameter of the pipe to be connected, and the surface assembly feature is determined based on the connection designs of the pipe). The inlet design will not be discussed in detail in this thesis, since this work mainly focus on the design of conformal cooling channels. To obtain the final MLACCD injection molding mold, Boolean operations are applied to combine the hose to the mold outline, and subtract the solid cooling channels as shown in Figure 2-4.

To summarize, a new conformal cooling design procedure is discussed in this chapter, since the conventional approach is no longer suitable for the MLACCD proposed in this work. There are four design steps to generate a MLACCD cooling system. First, the design parameters of a given conformal cooling topology are identified. Then, a supervised machine learning process is performed to obtain a surrogate model relating the identified design parameters and the resulting part surface temperature. Based on the surrogate temperature model, a temperature variance minimization (TVM) tool is developed to minimize the temperature variance of the part. Finally, the MLACCD mold can be generated based on the wireframe of the TVM cooling channels, and the mold outline. The detailed discussion of each step of the design process will be provided in Chapter 3 to Chapter 5, and validated by the case studies given in Chapter 6 . 


\section{CHAPTER 3 \\ Identification of design parameters and division of cooling surface}

In this chapter, the design parameters of the most suitable cooling channel topologies including zigzag, spiral, porous, and CPS are identified in Section 3.1 for the purpose of training data construction. Additionally, an effectiveness evaluation of the CPS design parameters in terms of their influences on the resulting part surface temperature is performed through the Moldflow Advisor $^{\circledR}$ simulations due to its novel design and unexplored influence of the design parameters on the cooling efficiency. In Section 3.2, a cooling surface division method is introduced for zigzag, spiral, and porous cooling channels, where each cooling topology is mapped to the part geometry to allow its largest optimization potentials. Since CPS is a unique type of porous structures that does not exhibit significant variations on its cooling performance for different geometrical designs of the part, it will not be considered in the division algorithm.

\subsection{Identification of design parameters}

In this section, the design parameters of zigzag, spiral, porous, and CPS cooling channels are selected to construct the training data sets in the machine learning process, to estimate the part surface temperature during the optimization process, and to define the cooling efficiency variation direction $\overrightarrow{\boldsymbol{C E V}}$ for the cooling surface division. Three rules need to be considered during the identification of the design parameters: (1) the design parameters should have the ability to effectively affect the cooling efficiency of the cooling system; (2) it should be practical to adjust the design parameters in the optimization process; (3) the design parameters should be essential in fully defining the geometrical design of the cooling channels. In Section 3.1.1, the identification of the design parameters for theoretically well-established conformal cooling topologies including zigzag, spiral, and porous designs is discussed. For CPS, the theoretical links between the design parameters and their resulting influence on the cooling efficiency is unclear. Thus, the 
effectiveness of the CPS design parameters needs to be evaluated, and the design parameter identification for CPS will be individually discussed in Section 3.1.2.

\subsubsection{Spiral, zigzag, and porous cooling systems}

As indicated in the heat transfer theory proposed by Xu et al. [10], the cooling efficiency of a spiral or zigzag conformal cooling channel is mainly affected by the half-part thickness $l_{p}$, coolant Reynold number Re, channel diameter $d$, cooling channel pitch width $W$, and cooling channel pitch to mold surface distance $l_{m}$ as shown in Figure 3-1. For porous conformal cooling channels, the most influential parameters besides $l_{p}$ are the porosity of the mold $\phi$, and the Reynold number Re. Among these parameters, $d, l_{m}$, and $\phi$ are the design parameters that are critical to fully

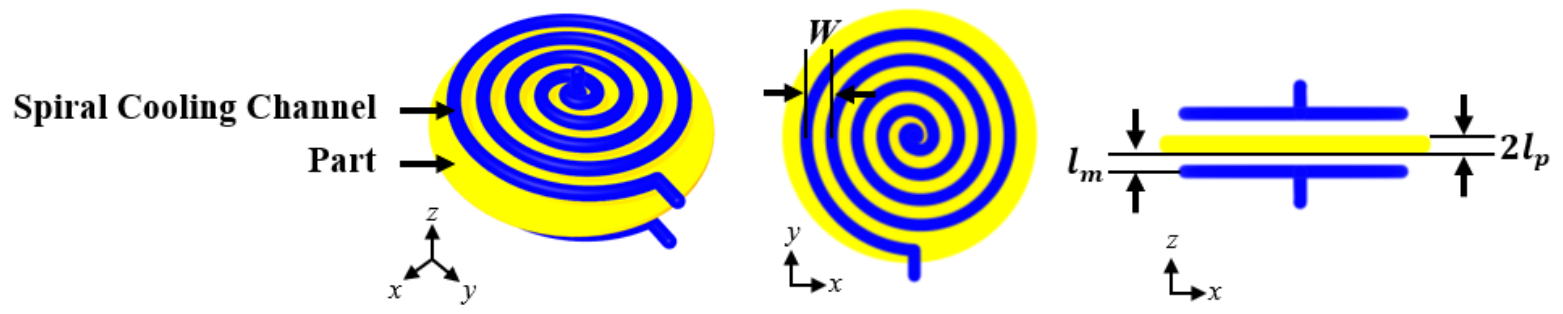

(a) Design parameters for spiral cooling channels

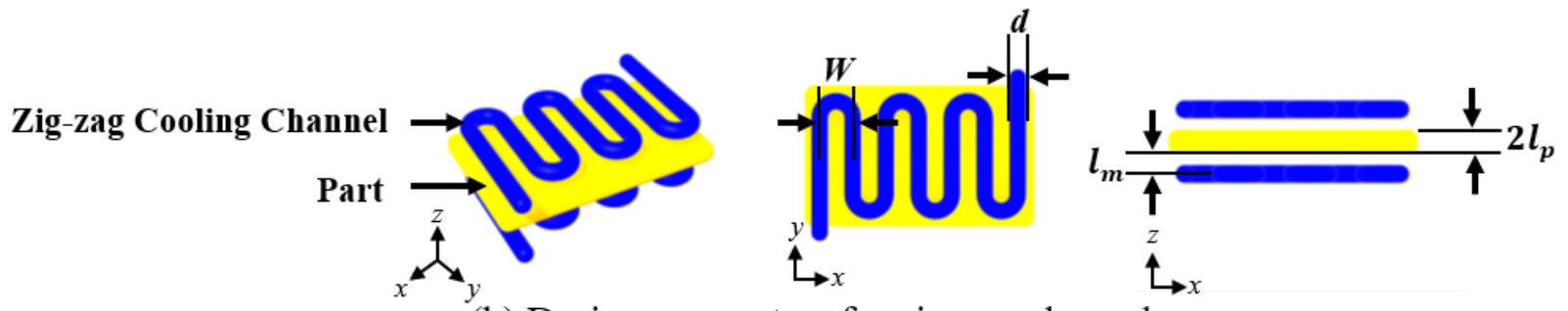

(b) Design parameters for zig-zag channels
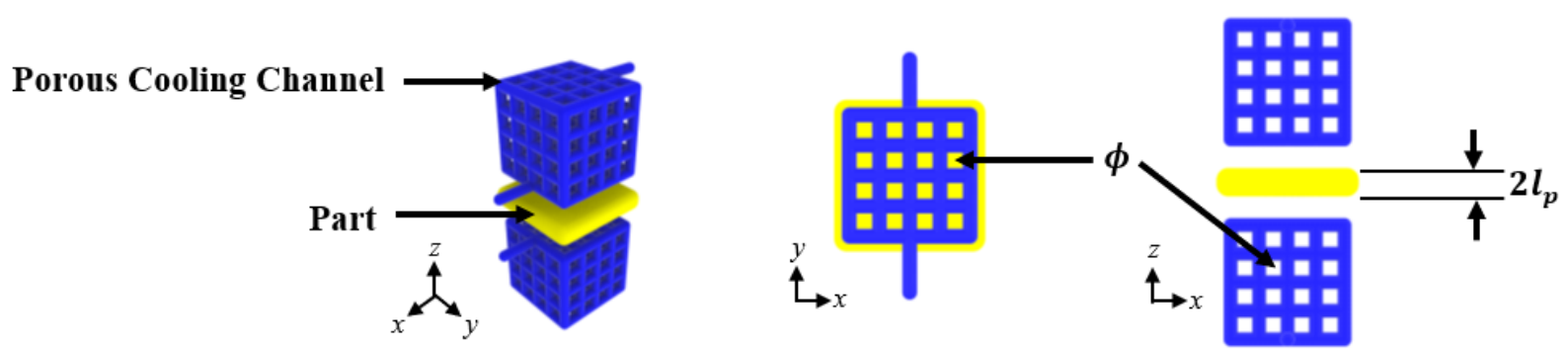

(c) Design parameters for porous channels

Figure 3-1 Design parameters for spiral, zigzag, and porous cooling channels 
define the geometrical designs of the cooling channels. However, there are two reasons that Re and $d$ are impractical to be varied during the optimization. First, the variation of Re requires designing multiple coolant inlets for different flow rates, which significantly increases the complexity of the cooling system. Second, the channel diameter $d$ is coupled with $l_{m}, W$, and Re.

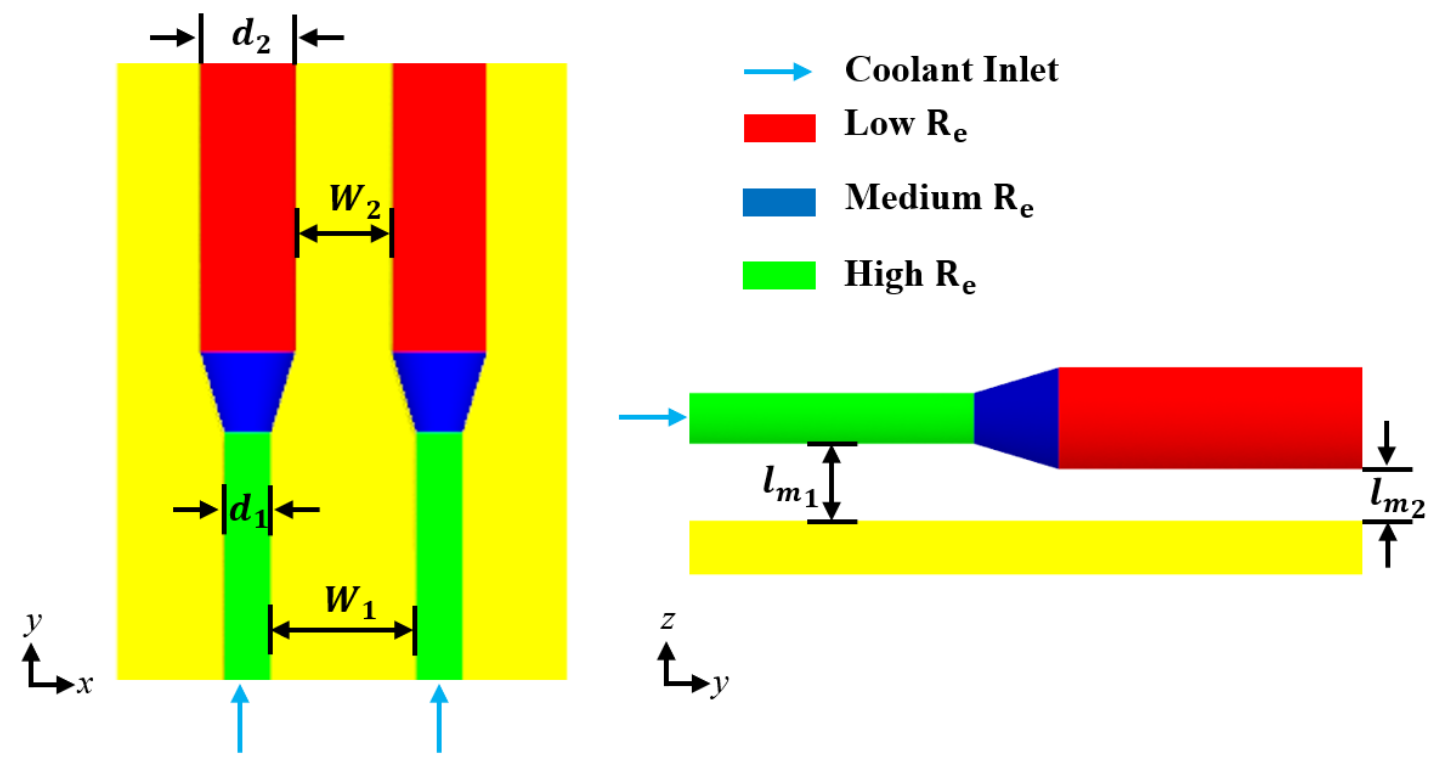

Figure 3-2. The coupling effect of changing the diameters of cooling channels

An example is shown in Figure 3-2 to explain the coupling effect of Re, $l_{m}, W$, and $d$. As the channel diameter varied from $d_{1}$ to $d_{2}$, the original pitch-to-pitch distance $W_{1}$ and pitch to mold surface distance $l_{m_{1}}$ are forced to be adjusted to $W_{2}$ and $l_{m_{2}}$ as given in equation (3-1) and equation (3-2):

$$
\begin{gathered}
W_{2}=W_{1}-\left(d_{2}-d_{1}\right) \\
l_{m_{2}}=l_{m_{1}}-\left(d_{2}-d_{1}\right)
\end{gathered}
$$

In addition, the mathematical relation between Re and $d$ for a circular channel is provided in equation (3-3): 


$$
\operatorname{Re}=\frac{Q}{\pi v d}
$$

where $Q$ is the volumetric flow rate, and $v$ represents the kinematic viscosity of the coolant. Therefore, increasing $d$ results in a reduction of Re as shown in Figure 3-2. These coupled effects make it very challenging to adjust $d$ during the optimization process. With these considerations and design parameters selection procedure, $W$ and $l_{m}$ are selected as the design parameters for spiral and zigzag cooling channels, and $\phi$ is selected as the design parameters for porous channels as shown in Figure 3-1.

\subsubsection{CPS}

To fully-define a three-layer CPS, the CPS design parameters have to be identified first. There are several reasons of selecting a three-layer CPS. First, additional cooling layers will have a much larger pitch to mold surface distance, which is not cost effective in terms of its cooling efficiency versus additional mass and manufacturing complexity. Second, recent publications have proven that two to three cooling layers will already produce a rapid cooling for the porous cooling channels

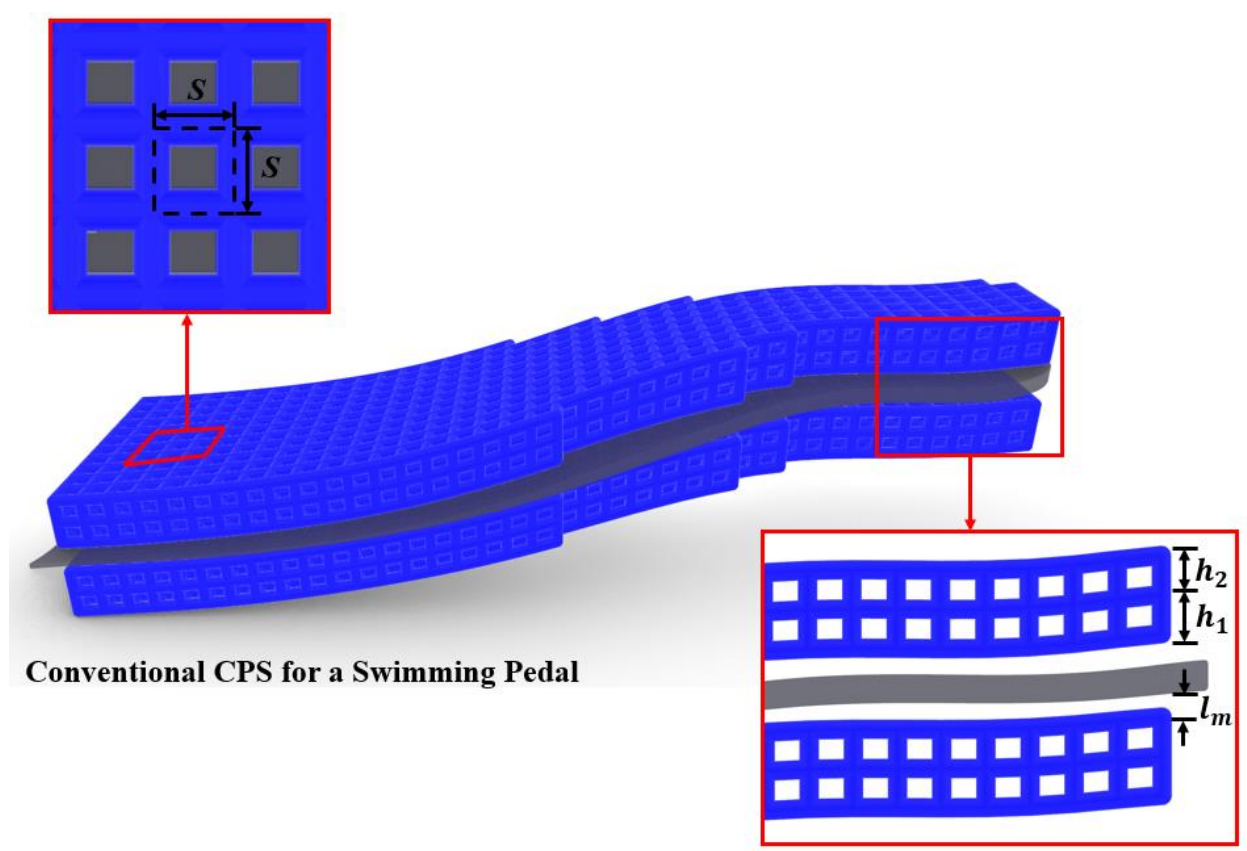

Figure 3-3. Required design parameters for a three-layer CPS 
$[13,21]$. As shown in Figure 3-3, four design parameters are needed to fully define a three-layer CPS: (1) CPS to mold surface distance $\boldsymbol{l}_{\boldsymbol{m}}$; (2) CPS cell size $S$; (3) distance between the first and the second cooling layers $\boldsymbol{h}_{\mathbf{1}}$; (4) distance between the second and the third cooling layers $\boldsymbol{h}_{\mathbf{2}}$.

(a) The simulation configuration for CPS trail simulations and training data collection

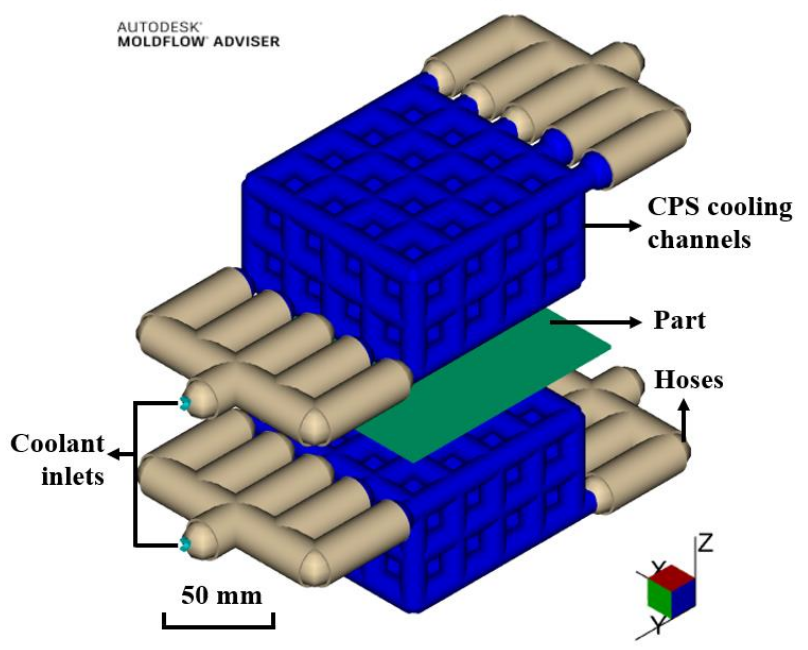

(b) Part temperature collection location

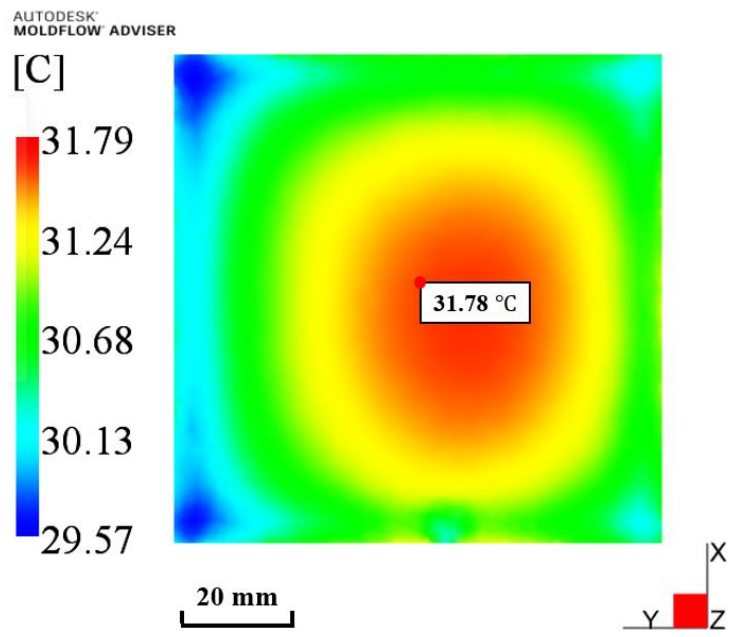

Figure 3-4. The simulation configuration and an example temperature collection for trial simulations and training data collection process

Table 3-1. Input parameters for cooling simulations

\begin{tabular}{|l|l|}
\hline Mold material & H13 Tool Steel \\
\hline Initial mold temperature & $323.15 \mathrm{~K}$ \\
\hline Part material & Generic PP \\
\hline Melt temperature & $493.15 \mathrm{~K}$ \\
\hline Coolant type & Water \\
\hline Coolant inlet temperature & $298.15 \mathrm{~K}$ \\
\hline Coolant flow rate & 20 lit/min \\
\hline Injection cycle time & 35 seconds \\
\hline CPS design parameters & {$\left[l_{m}, S, h_{1}, h_{2}\right]$} \\
\hline
\end{tabular}

Table 3-2. Range of the design parameters

\begin{tabular}{|l|l|}
\hline Design parameters & Range \\
\hline CPS to mold surface distance $l_{m}$ & $6 \mathrm{~mm} \sim 30 \mathrm{~mm}$ \\
\hline CPS cell size $S$ & $12 \mathrm{~mm} \sim 30 \mathrm{~mm}$ \\
\hline Distance between the first and the second cooling layers $h_{1}$ & $12 \mathrm{~mm} \sim 30 \mathrm{~mm}$ \\
\hline Distance between the second and the third cooling layers $h_{2}$ & $12 \mathrm{~mm} \sim 30 \mathrm{~mm}$ \\
\hline
\end{tabular}


Table 3-3. Effectiveness evaluation results for cps design parameters

\begin{tabular}{|c|c|c|c|c|}
\hline $\begin{array}{c}\text { Potential CPS } \\
\text { design parameters }\end{array}$ & $\begin{array}{l}\text { Parameter set } \\
{\left[\begin{array}{l}{\left[l_{m}, S, h_{1}, h_{2}\right]} \\
(\mathrm{mm})\end{array}\right.}\end{array}$ & $T\left({ }^{\circ} \mathrm{C}\right)$ & $\Delta T\left({ }^{\circ} \mathrm{C}\right)$ & Effectiveness evaluation \\
\hline \multirow[b]{2}{*}{$l_{m}$} & {$[6,12,12,12]$} & 28.6 & \multirow{2}{*}{3.5} & \multirow{2}{*}{ Effective } \\
\hline & {$[28,12,12,12]$} & 32.1 & & \\
\hline \multirow{2}{*}{$S$} & {$[6,12,12,12]$} & 28.6 & \multirow{2}{*}{1.9} & \multirow{2}{*}{ Effective } \\
\hline & {$[6,28,12,12]$} & 30.5 & & \\
\hline \multirow{2}{*}{$h_{1}$} & {$[6,12,12,12]$} & 28.6 & \multirow{2}{*}{0.2} & \multirow{2}{*}{ Not effective } \\
\hline & {$[6,12,28,12]$} & 28.8 & & \\
\hline \multirow{2}{*}{$h_{2}$} & {$[6,12,12,12]$} & 28.6 & \multirow{2}{*}{0} & \multirow{2}{*}{ Not effective } \\
\hline & {$[6,12,12,28]$} & 28.6 & & \\
\hline
\end{tabular}

Traditionally, the overall cooling performance of a CPS can be roughly modeled by the theoretical model derived previously in our lab [13]. However, whether the design parameters including $S, l_{m}, h_{1}$, and $h_{2}$ are effective in terms of affecting the resulting part surface temperature cannot be concluded from this model. Thus, the effectiveness if CPS design parameters in terms of cooling efficiency was not theoretically or numerically evaluated in previous publications, which is different from zigzag, spiral, and porous cooling systems as discussed before. Therefore, trail simulations are performed on Moldflow Advisor ${ }^{\circledR}$ with the input parameters provided in Table 3-1 to determine the effectiveness of these parameters in Figure 3-3 in terms of controlling the cooling efficiency, and the simulation configuration shown in Figure 3-4a. The resulting part surface temperature is observed as the CPS design parameters are set as their upper and lower limits, while the others remain unchanged. An example temperature collection is provided in Figure $3-4 \mathrm{~b}$. Note that only the temperature at the center of the part surface is collected, since the coolant flow rate of CPS is the most stable at that point. The range of these parameters are provided in Table 3-2 based on the feasible design area proposed by Xu et al. [10]. The input $\left[l_{m}, S, h_{1}, h_{2}\right]$ values together with the results of these trial simulations are provided in Table 3-3, where $\Delta T$ is the temperature difference between the simulation results of two parameter sets for each CPS 
design parameter. According to the effectiveness evaluation, the $h_{1}$ and $h_{2}$ are not effective in terms of influencing the cooling performance. Thus, the $l_{m}$, and $S$ are selected as the design parameters for CPS in this work, while the others are automatically set to their lower limits.

\subsection{Cooling surface division}

To achieve better cooling controllability and channel distribution flexibility during the design process of zigzag, spiral, and porous cooling channels and map the best cooling topology into different part regions, the cooling surface of the part is divided into three sub-regions. The CPS is not discussed in this section since it does not have large variations on its cooling performance for different part geometries. The separation of cooling surfaces is based on the comparison result between the cooling efficiency variation directions $\overrightarrow{C E V}$ of different cooling topologies as shown in Figure 3-5, and the thickness distribution of the cooling surface. The $\overrightarrow{C E V}$ is defined as the direction that the local cooling efficiency of the channels which can be varied through locally adjusting the conformal cooling design parameters including $W, l_{m}$, and $\phi$, where the local cooling efficiency is the cooling rate on the part surface below a specific region of the cooling channels. For spiral cooling channels as shown in Figure 3-5a, variation of the spiral pitch-topitch distance $W_{s i j}$ and pitch to mold surface distance $l_{m s i j}$ provides the cooling controllability on the direction perpendicular to the channel distribution direction $\vec{c}_{s}$. Thus, the cooling efficiency variation directions of the spiral channels $\overrightarrow{C E V_{s l}}$ is defined in equation (3-9):

$$
\overrightarrow{C E V_{s l}} \in\left\{\overrightarrow{C E V} \mid \overrightarrow{C E V} \perp \vec{c}_{s}\right\}
$$

Obviously, the pattern of $\overrightarrow{C E V_{s l}}$ as shown in Figure 3-5a matches to a centripetal thickness variation, which results in a closed and circular thickness contour line on the part geometry. For zigzag cooling channels as shown in Figure 3-5b, the cooling channels are mainly distributed along $\pm \vec{y}$. In addition, the variation of both $W_{z i}$ and $l_{m z i}$ contributes to the cooling controllability on 


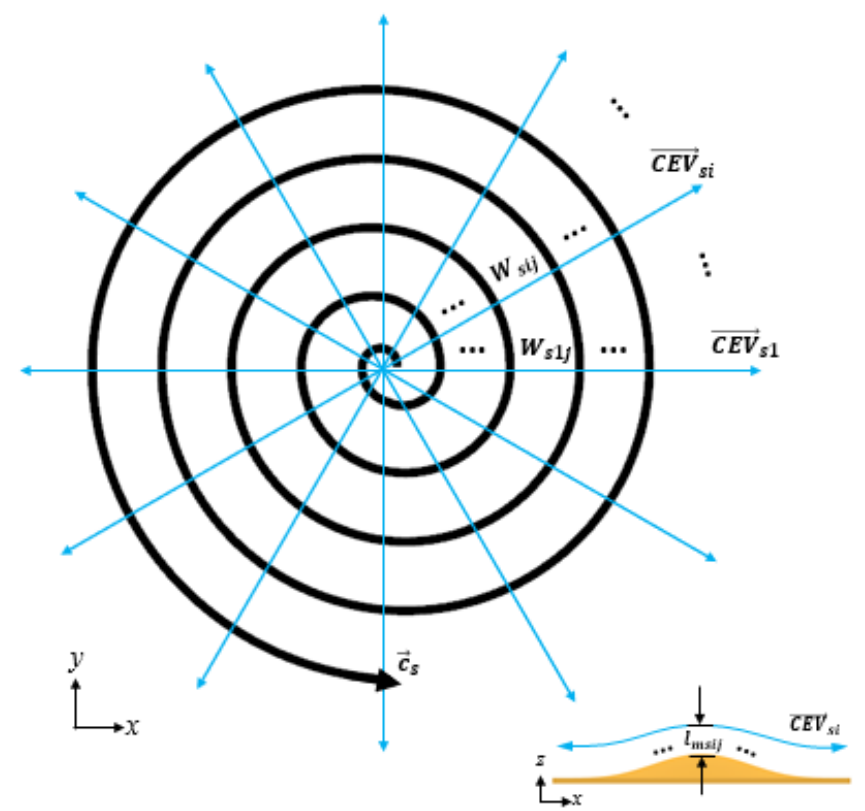

(a) Spiral $\overrightarrow{C E V}$ pattern
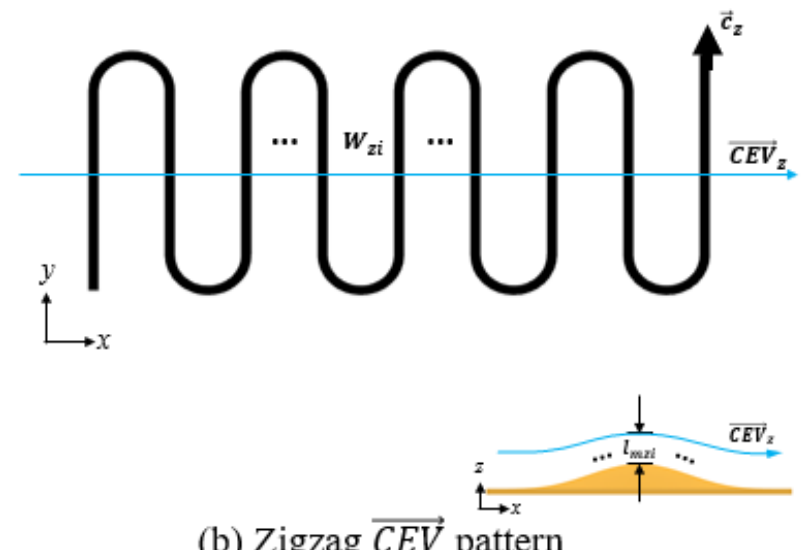

(b) Zigzag $\overrightarrow{C E V}$ pattern

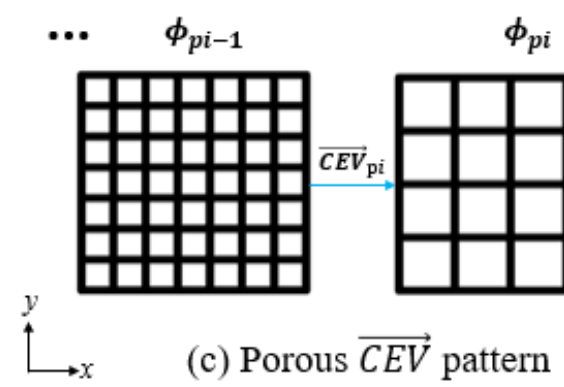

Figure 3-5. The cooling efficiency variation directions $\overrightarrow{C E V}$ for each cooling channel design the direction vertical to $\vec{c}_{z}$ similar to the spiral cooling channels. Hence, the cooling efficiency variation directions of the zigzag channels $\overrightarrow{C E V_{z}}$ is defined in equation (3-10): 


$$
\overrightarrow{C E V_{z}} \in\{\overrightarrow{C E V} \mid \overrightarrow{C E V} \perp \vec{y}\}
$$

Different from spiral cooling channels, the pattern of $\overrightarrow{C E V_{z}}$ matches with a unidirectional thickness variation, which has an open contour line of part thickness. For porous cooling channels, the porosity $\phi_{i}$ varies regionally as shown in Figure 3-5c. Therefore, the pattern of $\overrightarrow{C E V_{p}}$ contains the vectors $\overrightarrow{C E V_{p l}}$ pointing from region $i-1$ to region $i$, where each region $i$ exhibits a uniform

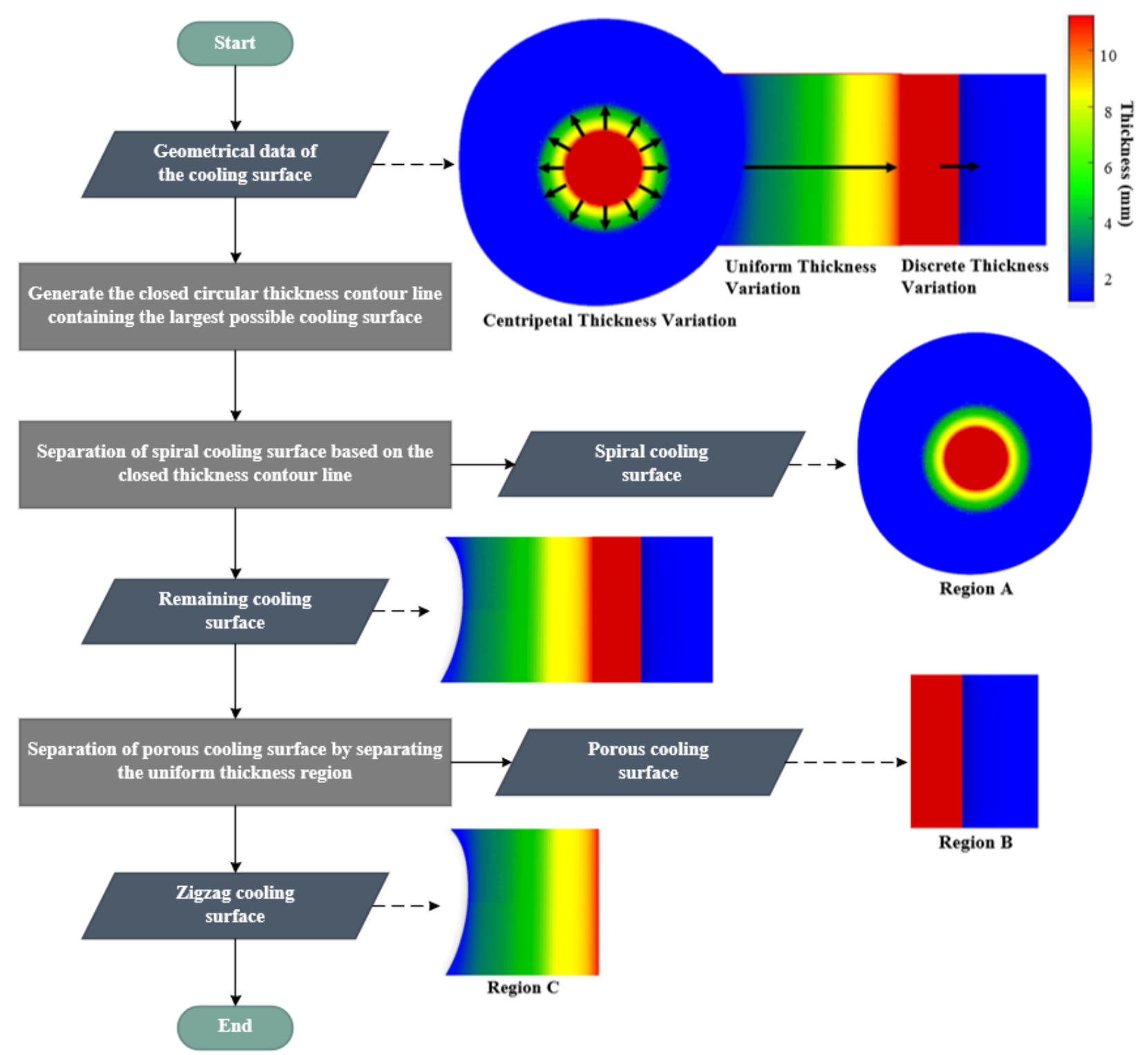

Figure 3-6. General procedure of the cooling surface division 
thickness value. Thus, $\overrightarrow{C E V_{p}}$ matches to a discrete thickness variation of part geometry, which refers to uniform thickness regions.

There are three steps to achieve the cooling surface division as shown in Figure 3-6. First, the closed circular thickness contour line is derived containing the largest possible cooling area. This contour line represents the boundary of the largest sub-regions with centripetal thickness variation, and hence it is the dividing line for the spiral cooling surface. Second, the spiral cooling surface is separated from the total cooling surface based on the closed circular contour line derived. Then, the porous cooling region is divided from the rest of the total cooling surface by separating the sub-regions with uniform thickness values, and the remaining cooling surface after the separation represents the zigzag cooling region.

\subsection{Summary}

In this chapter, the identification of design parameters for conformal cooling topologies including zigzag, spiral, porous, and CPS are discussed. In addition, a cooling surface division method is proposed to provide a better cooling controllability and channel distribution flexibility for the situation where multiple cooling channel topologies are applied for a same part. Based on the design parameter selection principles proposed at the beginning of this chapter, $\left[W, l_{m}, l_{p}\right],[\phi$, $\left.l_{p}\right],\left[S, l_{m}, l_{p}\right]$ are identified as the design parameters for zigzag and spiral, porous, and CPS cooling channels, respectively. Using the cooling surface division method, a given part geometry can also be divided into sub-regions for zigzag, spiral, and porous cooling channels based on the comparison result between the $\overrightarrow{C E V}$ patterns of the part and the cooling topologies. The identified design parameters are then applied to generate the training data during the machine learning process, which will be discussed in the next chapter. 


\section{CHAPTER 4 \\ Machine learning prediction of part surface temperature}

In this chapter, the machine learning process is carefully discussed, where in total four conformal cooling topologies including CPS, zigzag, spiral, and porous structures are analyzed using the supervised machine learning method. A general description of the machine learning procedure is provided in Figure 4-1. To generate the training data, algorithms are first designed to create the Moldflow Advisor ${ }^{\circledR}$ simulation files for these cooling channel topologies. Specifically, these programs generate the simulation files that can be executed by Moldflow Advisor ${ }^{\circledR}$ with all the possible combination of the cooling channel design parameters for every possible $l_{p}$ value. The part surface temperatures are then collected from the cooling simulation results with respect to different sets of the design parameters, where these temperature data are combined with their corresponding design parameters as the training data. The training data are then fed into the supervised machine learning algorithm which is a two-layer ANN, where the detailed architecture of the ANN will be discussed in Section 4.2.1. Within the ANN, the input layer contains all the design parameters, and the output layer is the resulting temperature value. As a result, a trained surrogate temperature model is generated. To evaluate the model, the loss function and the maximum temperature difference between the test data and the predicted data are calculated. If the loss function has a steady decrease, and the maximum model error is acceptable, the surrogate model is considered to be ready for being applied during the optimization process. Otherwise, revisions are required for the program parameters of ANN to decrease the loss during the training, and more training data have to be generated and fed into the ANN to improve the model accuracy. In this chapter, the detailed explanation of the training data construction for these topologies is given in Section 4.1. In Section 4.2, the ANN training process, and the evaluation results of the trained machine learning surrogate temperature model are provided. 


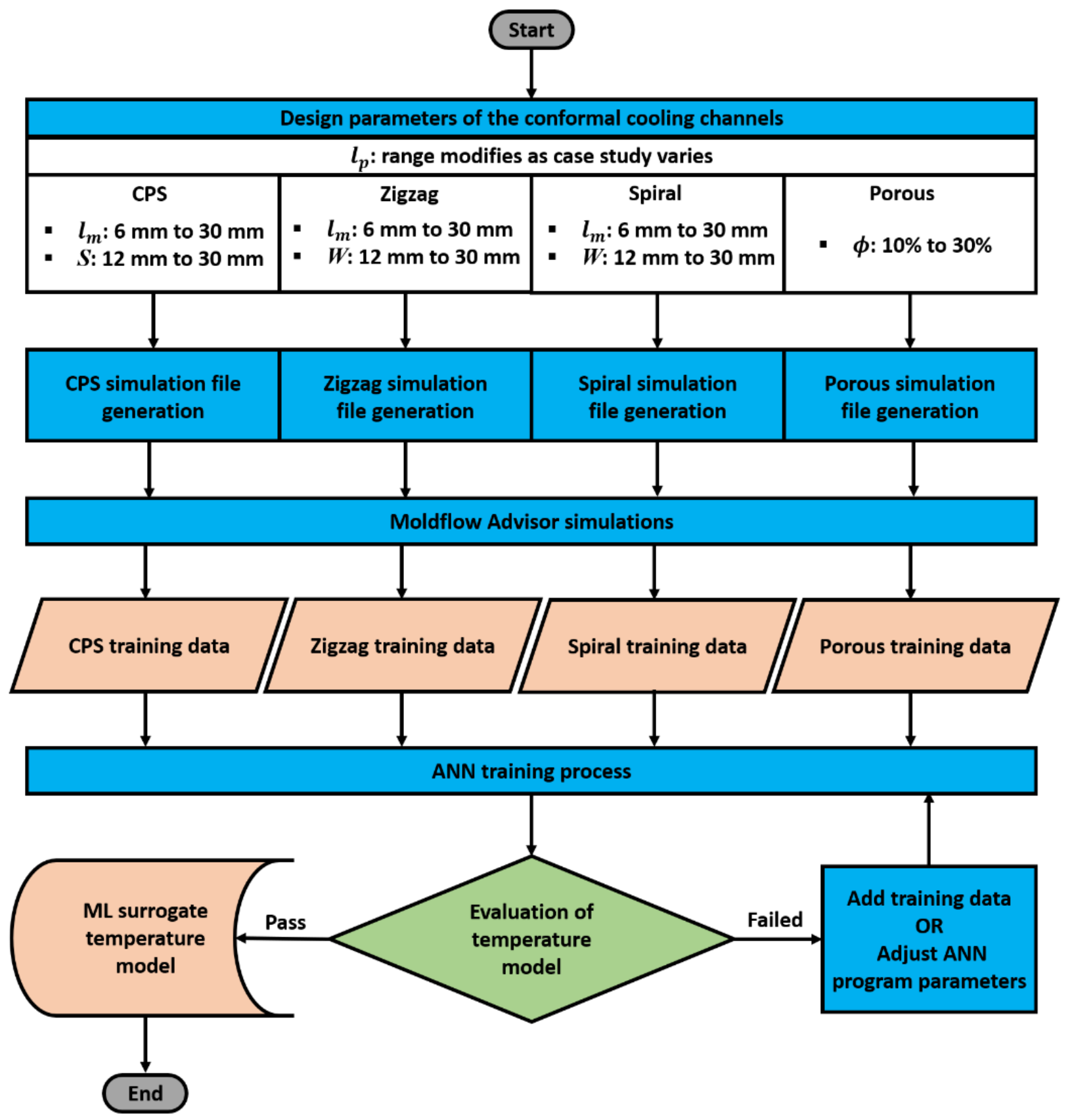

Figure 4-1. The machine learning procedure

\subsection{Generation of training data}

In this section, the algorithms that generates the training data simulation files of CPS, zigzag, spiral, and porous conformal cooling topologies are given in Section 4.1.1 to 4.1.3. The simulation 
configuration for the temperature data collection of each cooling topology is also provided in these sections and discussed carefully.

\subsubsection{Algorithm design for the generation of zigzag cooling channels}

The flowchart for the zigzag cooling channels generation program is shown in Figure 4-2. The inputs of this program are lists of $W, l_{m}$, and $l_{p}$ values containing all the possible design parameter values that the defined by the user for the training process. With given lists of $W, l_{m}$, and $l_{p}$ values

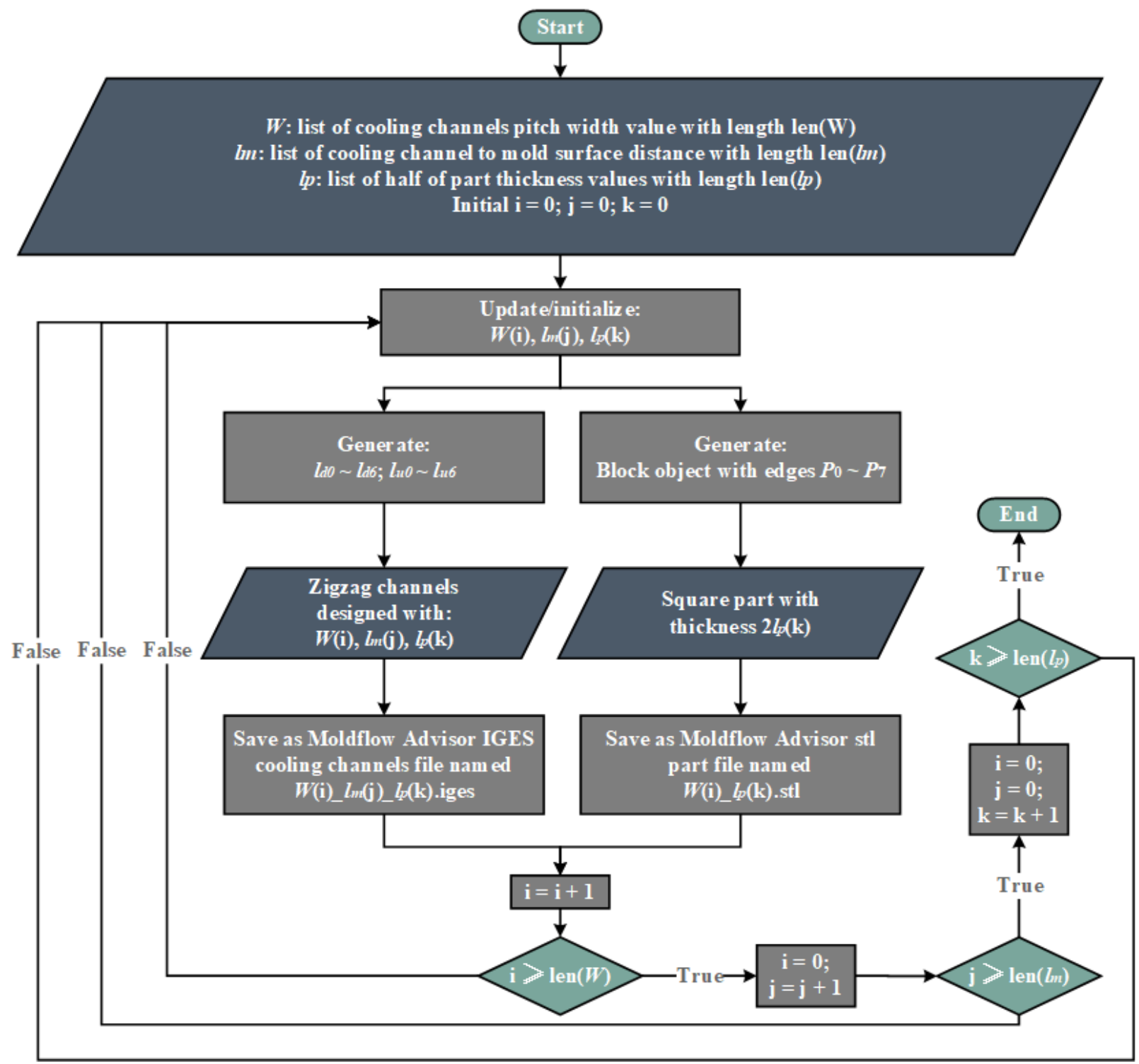

Figure 4-2. The zigzag simulation files generation algorithm 
and the list positions $\mathrm{i}, \mathrm{j}$, and $\mathrm{k}$, the program generates two files: (1) a rectangular part with the thickness of $2 l_{p}(\mathrm{k})$ designed for the cooling channels with the width of $W(\mathrm{i}) ;(2)$ the control lines of the cooling channels with a pitch-to-pitch width of $W(\mathrm{i})$, and a distance of $l_{m}(\mathrm{j})$ to surface of

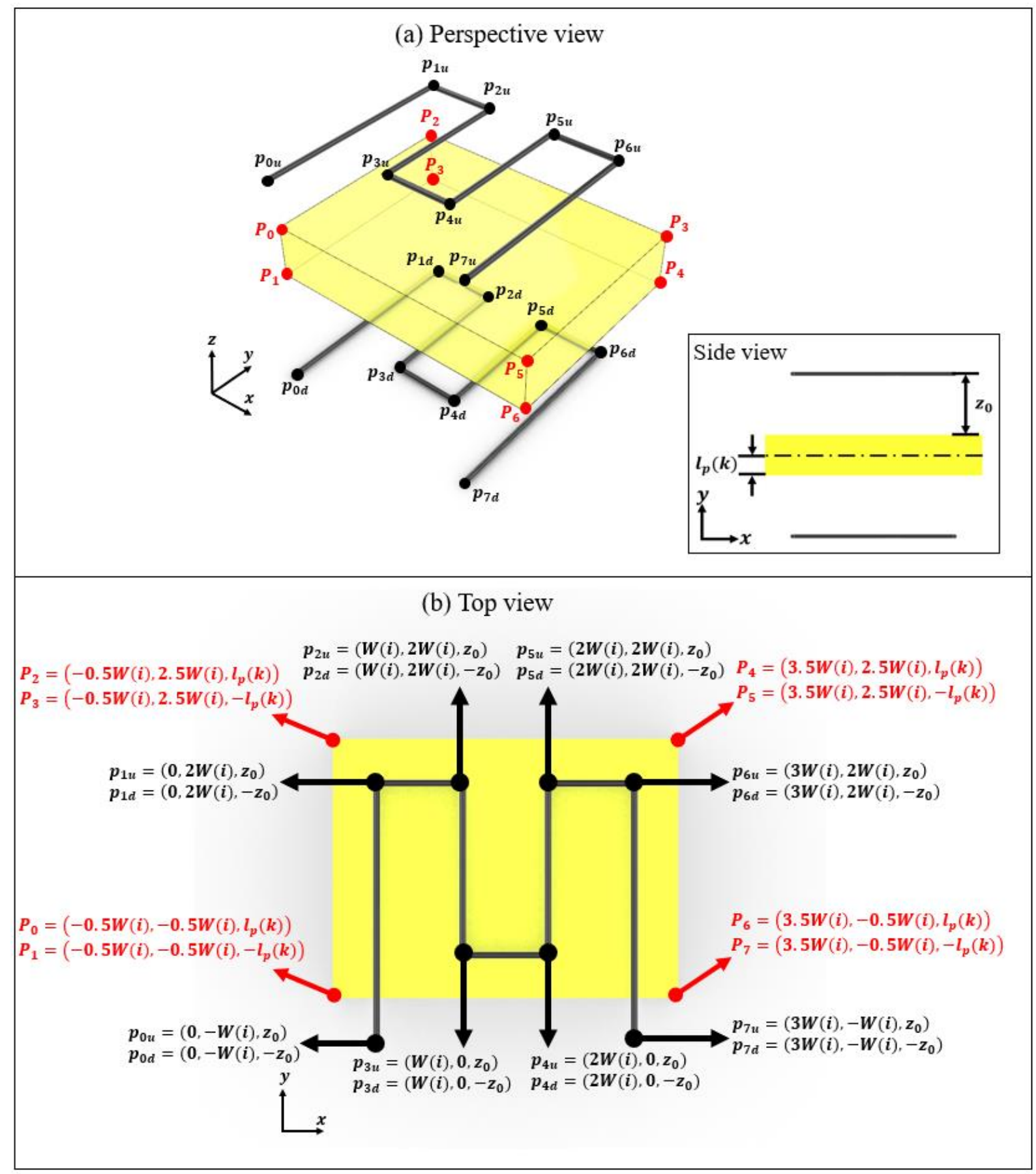

Figure 4-3. The coordinates of the cooling channel control points and the block object edges 
the part with the thickness of $l_{p}(\mathrm{k})$. Specifically, the control point coordinates of the cooling channel control lines, and the edge coordinates of the part is provided in Figure 4-3. The generated cooling channel file and the part file are saved with the names of $W(\mathrm{i})_{-} l_{m}(\mathrm{j})_{-} l_{p}(\mathrm{k})$.iges and $W(\mathrm{i}) \_l_{p}(\mathrm{k})$. stl, respectively. This process is iterated by updating the values of $\mathrm{i}, \mathrm{j}, \mathrm{k}$ so that every possible combinations of $W(\mathrm{i}), l_{m}(\mathrm{j}), l_{p}(\mathrm{k})$ are accessed as shown in Figure 4-2.

Table 4-1. Cooling simulations input parameters for training data collection

\begin{tabular}{|l|l|l|}
\hline Topology & Zigzag and spiral & Porous and CPS \\
\hline Mold material & H13 Tool Steel & H13 Tool Steel \\
\hline Initial mold temperature & $323.15 \mathrm{~K}$ & $323.15 \mathrm{~K}$ \\
\hline Part material & Generic PP & Generic PP \\
\hline Melt temperature & $493.15 \mathrm{~K}$ & $493.15 \mathrm{~K}$ \\
\hline Cooling channel diameter & $6 \mathrm{~mm}$ & $6 \mathrm{~mm}$ \\
\hline Coolant type & Water & Water \\
\hline Coolant inlet temperature & $298.15 \mathrm{~K}$ & $298.15 \mathrm{~K}$ \\
\hline Coolant flow rate & 5 lit/min & 5 lit/min \\
\hline Injection cycle time & 35 seconds & 35 seconds \\
\hline
\end{tabular}
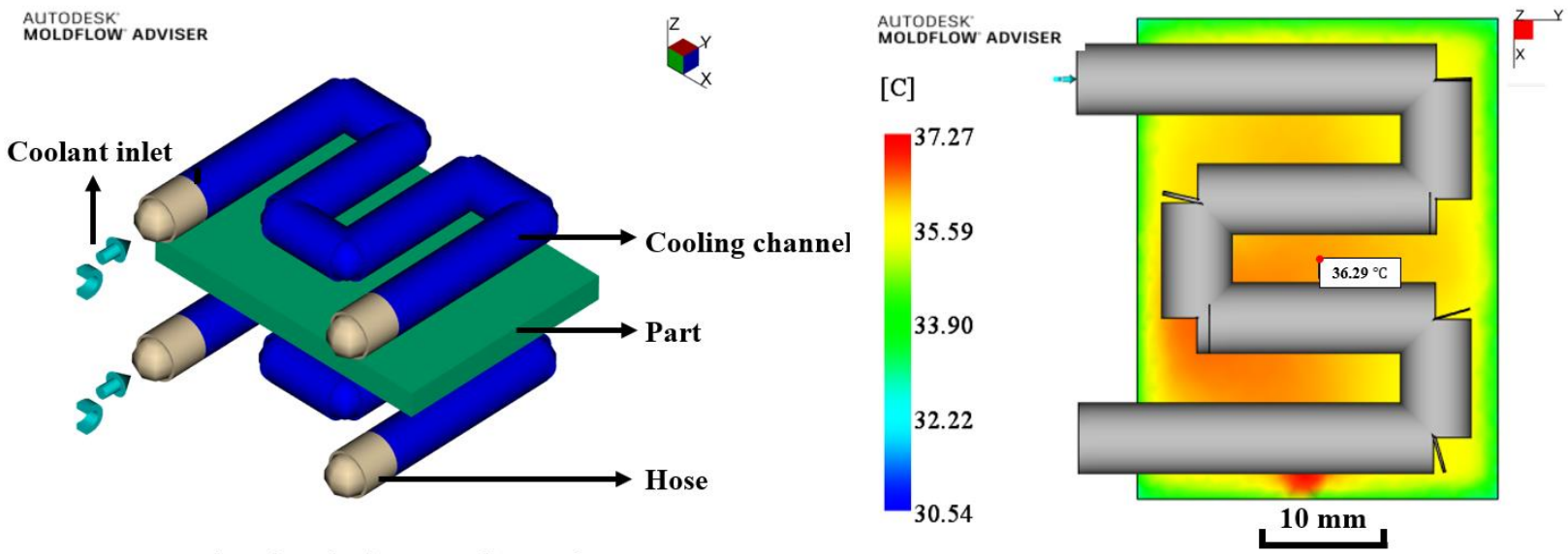

(a) The simulation configuration for zigzag training data collection

(b) Part temperature collection location

Figure 4-4. Example simulation configuration and temperature distribution result for zigzag cooling channels

Once the simulation files are generated, the Moldflow Advisor ${ }^{\circledR}$ cooling simulation is performed with the input parameters as shown in Table 4-1. Figure 4-4a shows an example of a 
simulation set up with the imported zigzag cooling channels and block-shaped part, where Figure 4-4b shows the part temperature distribution of the cooling simulation. From the result of each simulation, a corresponding temeprature of the part is collected for each set of the design parameters, and will be finally used in the ANN training process.

\subsubsection{Algorithm design for the generation of spiral cooling channels}

To understand the overall spiral cooling channels generation algorithm, a simplified version of the spiral boundary distance mapping (BDM) strategy proposed by Wang et al. [14] needs to be first explained. Figure 4-5 shows the procedure of creating spiral cooling channels based on a given

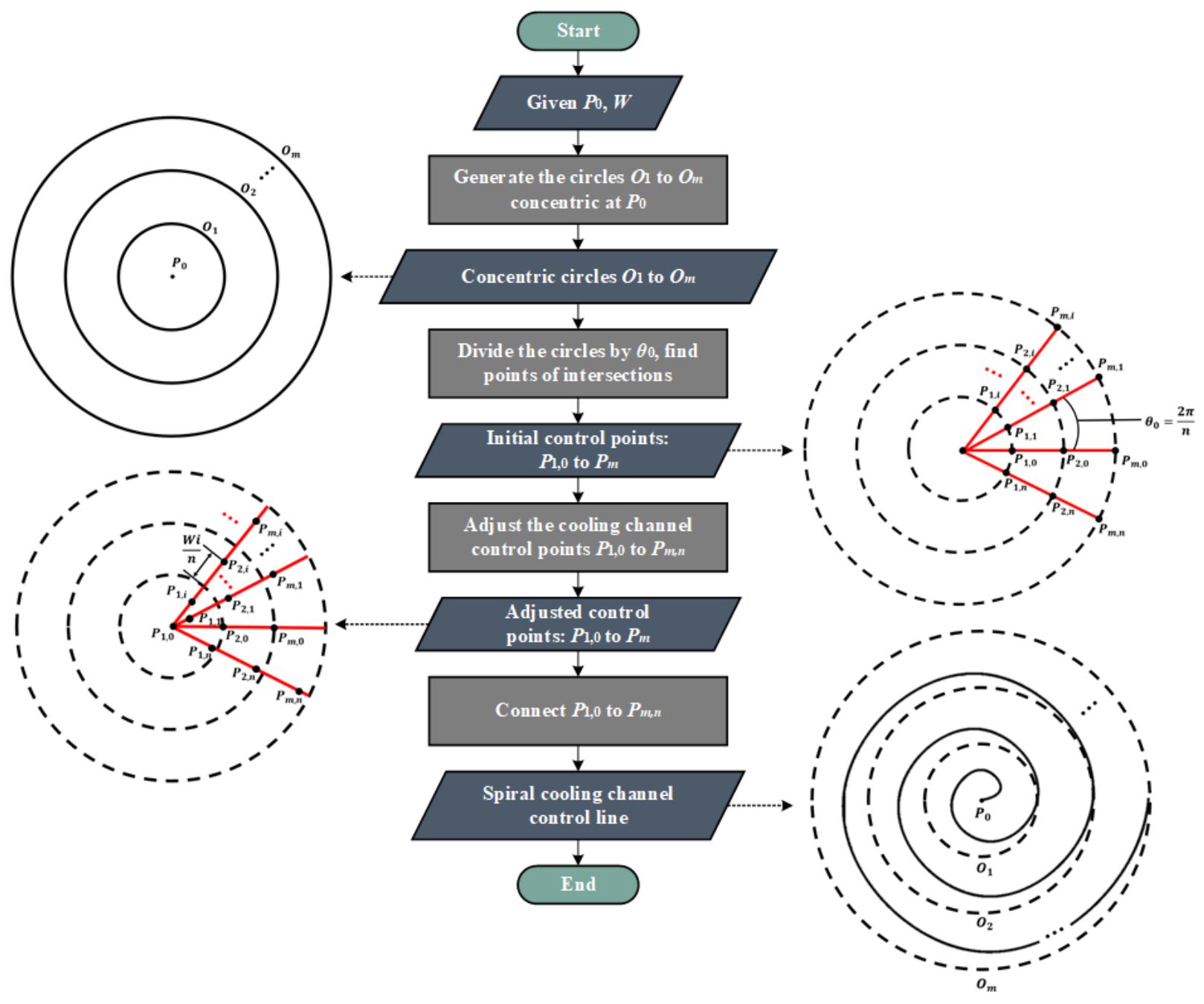

Figure 4-5. Simplified spiral BDM method 
start point $P_{0}$, and $W$ value. To create a cooling channel control line with $m$ helixes separated by the pitch width $W$, circles $O_{1}$ to $O_{m}$ concentric at $P_{0}$ are designed with the radius $W$ to $m W$. These circles are then divided into $n+1$ arcs by angle $\theta_{0}=\frac{2 \pi}{n}$, where $n$ is the resolution for the division. As a result, points of intersections $P_{1,0}$ to $P_{m, n}$ are extracted, where $P_{i, j}$ represents the intersection between the $i^{\text {th }}$ circle and $j^{\text {th }}$ division line. Afterwards, the spiralization technique is applied to generate the spiralized control points. To illustrate, every points of intersections $P_{1, i}$ to $P_{m, i}$ are adjusted by a distance $\frac{W i}{n}$ on the division line as shown in Figure 4-5. For each $O_{a}$ to $O_{a+1}$, a smooth connection is formed through connecting the adjusted $P_{a, 0}$ to $P_{a, n}$. Finally, all the adjusted points are connected with the sequence provided in equation (4-1), and the resulting spiral cooling channels are formed. For the training data collection purposes, the simulation file should be as simple and straightforward as possible to improve the data collection efficiency. Thus, the part files as shown in Figure 4-6a are generated based on the design of spiral cooling channels. The disk-shaped parts are chosen because it not only reduces the modelling and design efforts, but also decreases the simulation time.

$$
P_{1,0} \rightarrow P_{1,1} \rightarrow \cdots \rightarrow P_{1, n} \rightarrow P_{2,0} \rightarrow \cdots \rightarrow P_{2, n} \rightarrow \cdots \rightarrow P_{m, 0} \rightarrow \cdots \rightarrow P_{m, n}
$$

The overall flowchart explaining the generation of the spiral simulation files is provided in Figure 4-7. Basically, the program is designed to iterate over all combinations of $W, l_{m}$, and $l_{p}$ values in a similar way as zigzag simulation file generation program, and generates the spiral simulation files. Note that the z-coordinates of $P_{0}$ for the upper and lower spiral cooling channels are set as $\pm\left(l_{m}+l_{p}\right)$ as shown in Figure 4-6b. This distance makes sure that the spiral cooling channels for a part of $2 l_{p}$ thickness exhibit a pitch to mold surface distance of $l_{m}$. The number of 


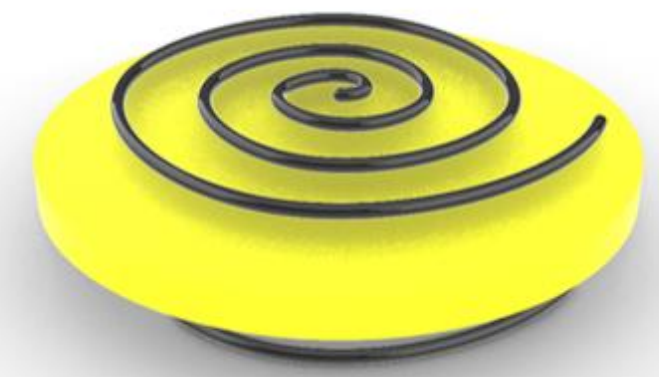

(a) Perspective view of the disk-shaped part design

$$
\overrightarrow{l_{m}}+\overline{l_{p}} \underline{\underline{\beth}}
$$

(b) The relative positions of the part and the channel control lines

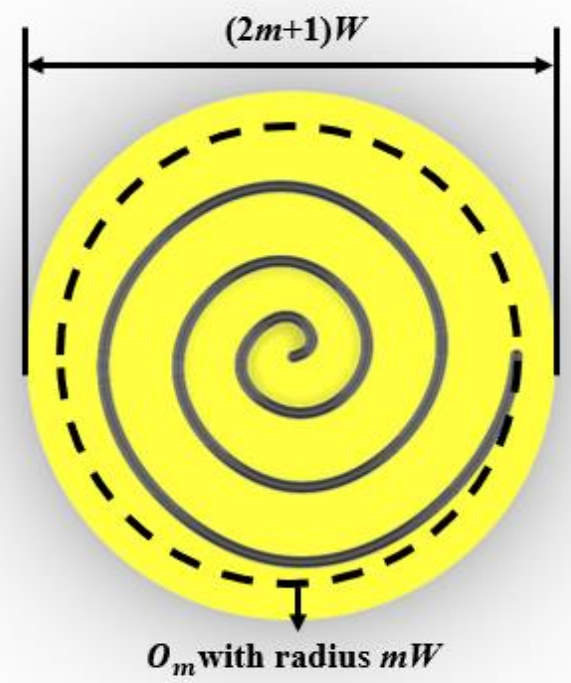

(c) Diameter of the disk-shaped part

Figure 4-6. The part design and the relative part to channels positions

the initial concentric circles are set to $m=3$ during the iteration, where the radius of the diskshaped part is automatically designed as $1.5 \mathrm{~W}$ following the calculation provided in Figure 4-6c. There are three reasons of setting $m$ to 3: (1) for any $m>3$, the simulation time significantly increase, which increase the overall training data collection time; (2) for any $m>3$, the coolant 


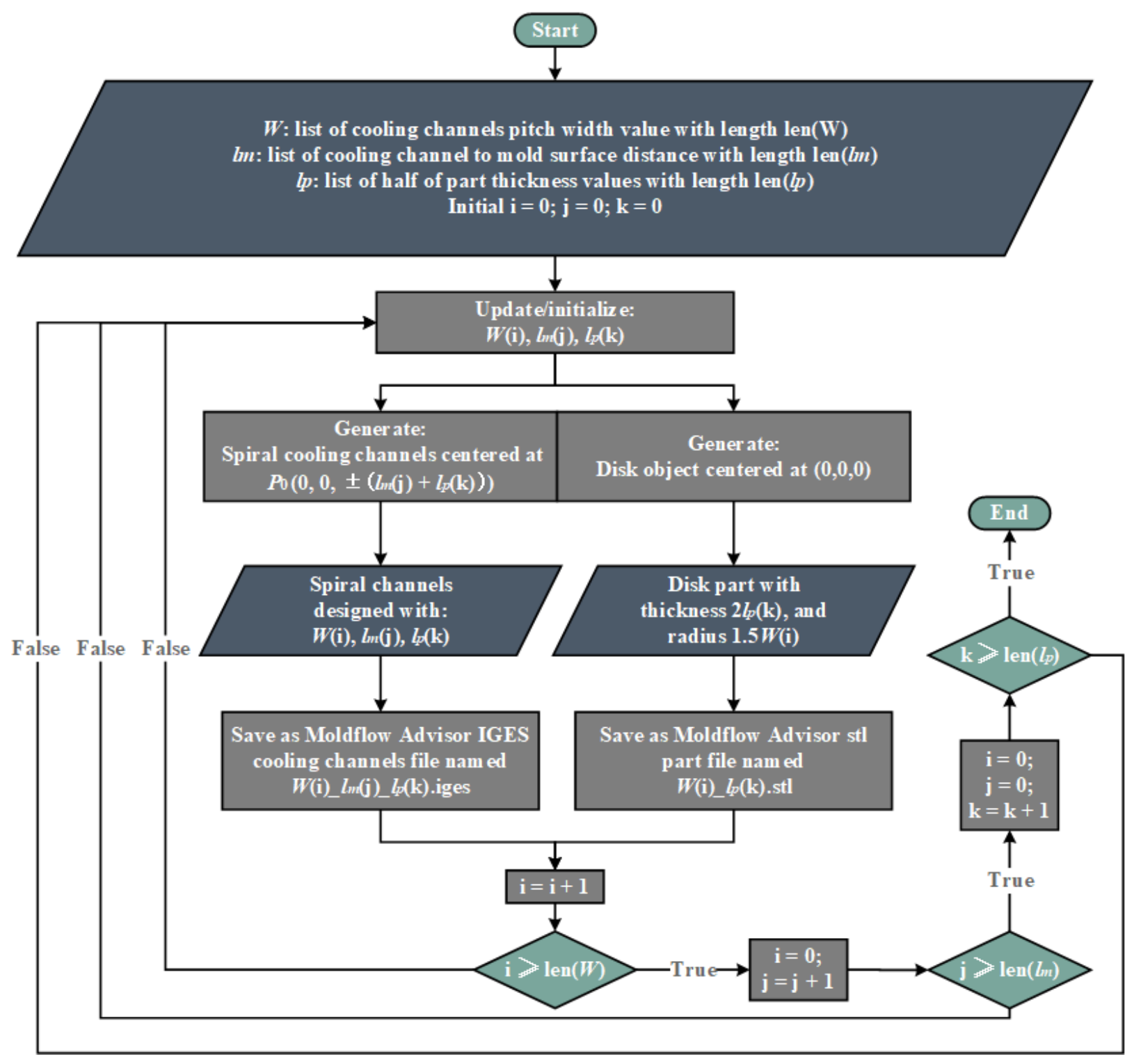

Figure 4-7. The spiral simulation files generation algorithm

will have a non-negletable temperature raise, which results in a reduction of the training data accuracy; (3) for any $m<3$, the temperature collection point will be too close to either the inlets or the outlets of the coolant, which negatively affect the training data accuracy. Figure 4-8a provides the simulation configuration for the training data collection of spiral conformal cooling channels with the input parameters provided in Table 4-1, where Figure 4-8b shows the simulation 
result. Based on the simulation result, the resulting part surface temperature is finally collected for the ANN training process.

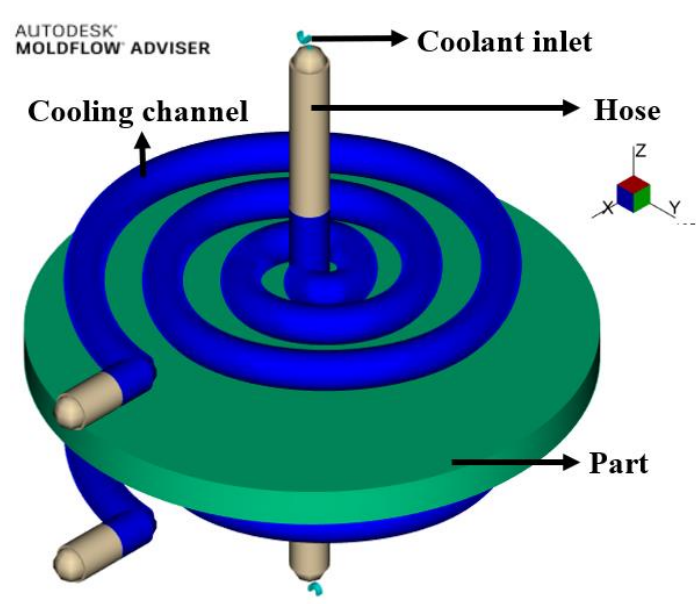

(a) The simulation configuration for spiral training data collection

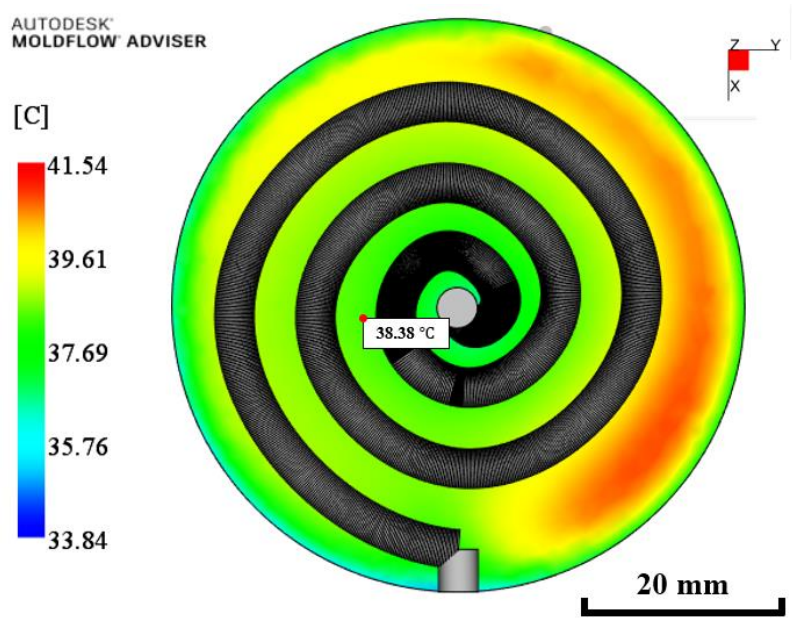

(b) Part temperature collection location

Figure 4-8. Example simulation configuration and temperature distribution result for spiral cooling channels

\subsubsection{Algorithm design for generation of porous and CPS cooling channels}

The algorithms that generate the porous and CPS simulation files for training data collection purposes are discussed together in this section due to similarities of porous and CPS in terms of their channel topologies. Based on a given set of design parameters, Figure 4-9 and Figure 4-10 provide the flowcharts for the generation of CPS cooling channels, porous cooling channels, and their correpsonding parts. For CPS cooling channels, the upper and lower initial cooling planes are first generated based on given $l_{m}, l_{p}$, and $S$ values, where the coordinate of the node on $i^{\text {th }}$ row and $j^{\text {th }}$ column of the initial cooling plane is derived in equation (4-2):

$$
P_{0(i, j)}=\left(i S, j S, \pm\left(l_{m}+l_{p}\right)\right)
$$

where $P_{0(i, j)}$ represents the node intersected by $i^{\text {th }}$ row and $j^{\text {th }}$ column of the initial cooling planes, the \pm sign indicates that in total two initial cooling planes are generated, which are positioned above and below the part object as shown in Figure 4-9. To construct three layers of the cooling 


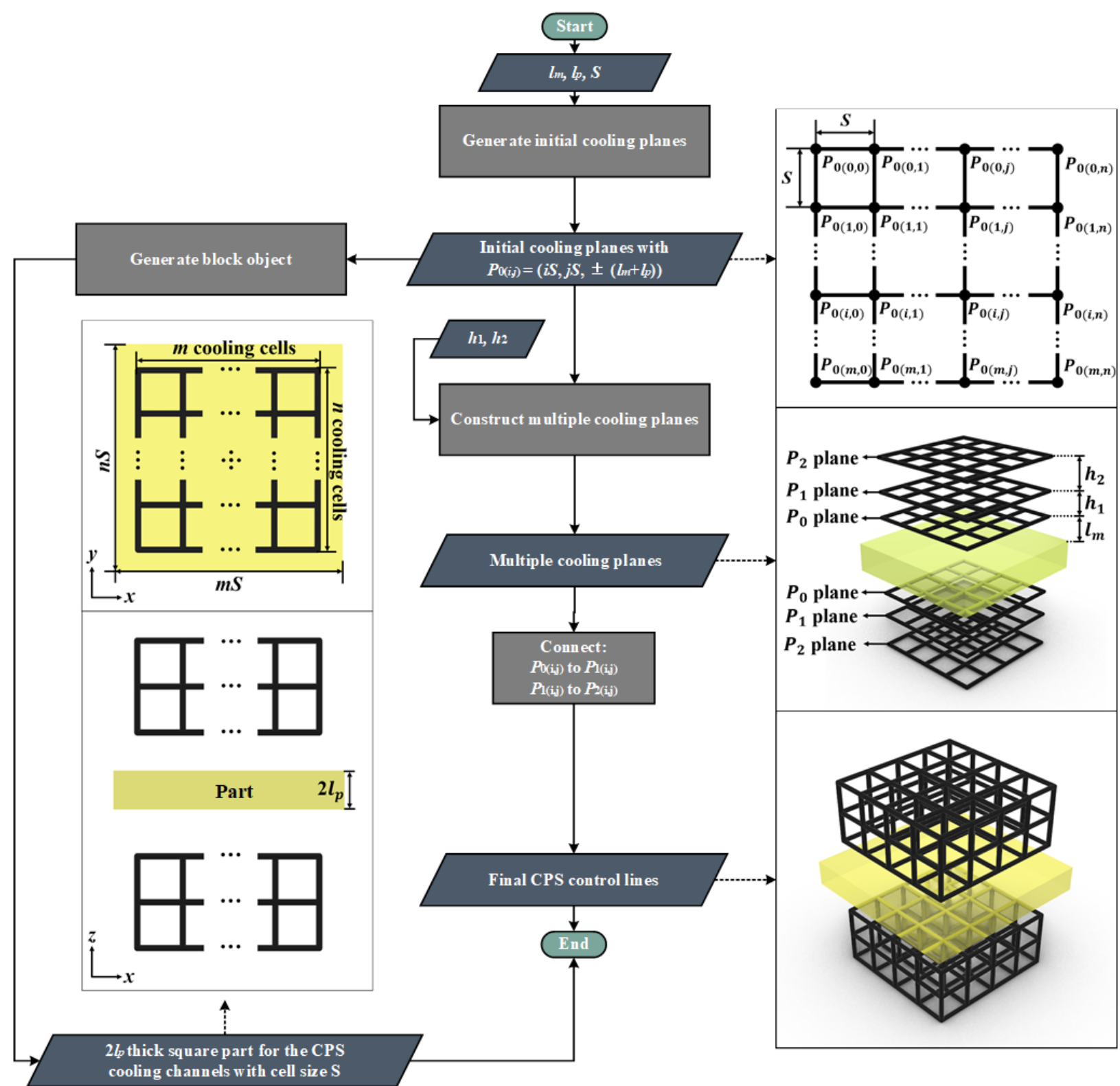

Figure 4-9. The CPS cooling channels generation method

surfaces, these cooling planes are then copied and lifted from the part surface with the distance $h_{1}$ and $h_{1}+h_{2}$ as shown in Figure 4-9. Connection lines are then formed between each $P_{0(i, j)}, P_{1(i, j)}$ and $P_{1(i, j)}, P_{2(i, j)}$ pairs so that the final CPS cooling channels are formed for the training data collection purposes. A block-shaped object with the thickness of $2 l_{p}$ is designed as the part for the training data simulation due to its simple geometry, which reduces the simulation time. The 
width of the part is set to be $m S$ and $n S$ for the cooling planes of $m \times n$ size, since these width values can provide a porper $0.5 \mathrm{~S}$ edge distance (this distance ensures the edge of the part be porperly but not over cooled, which affects simulation accuracy) between the edge of cooling channels and the part.

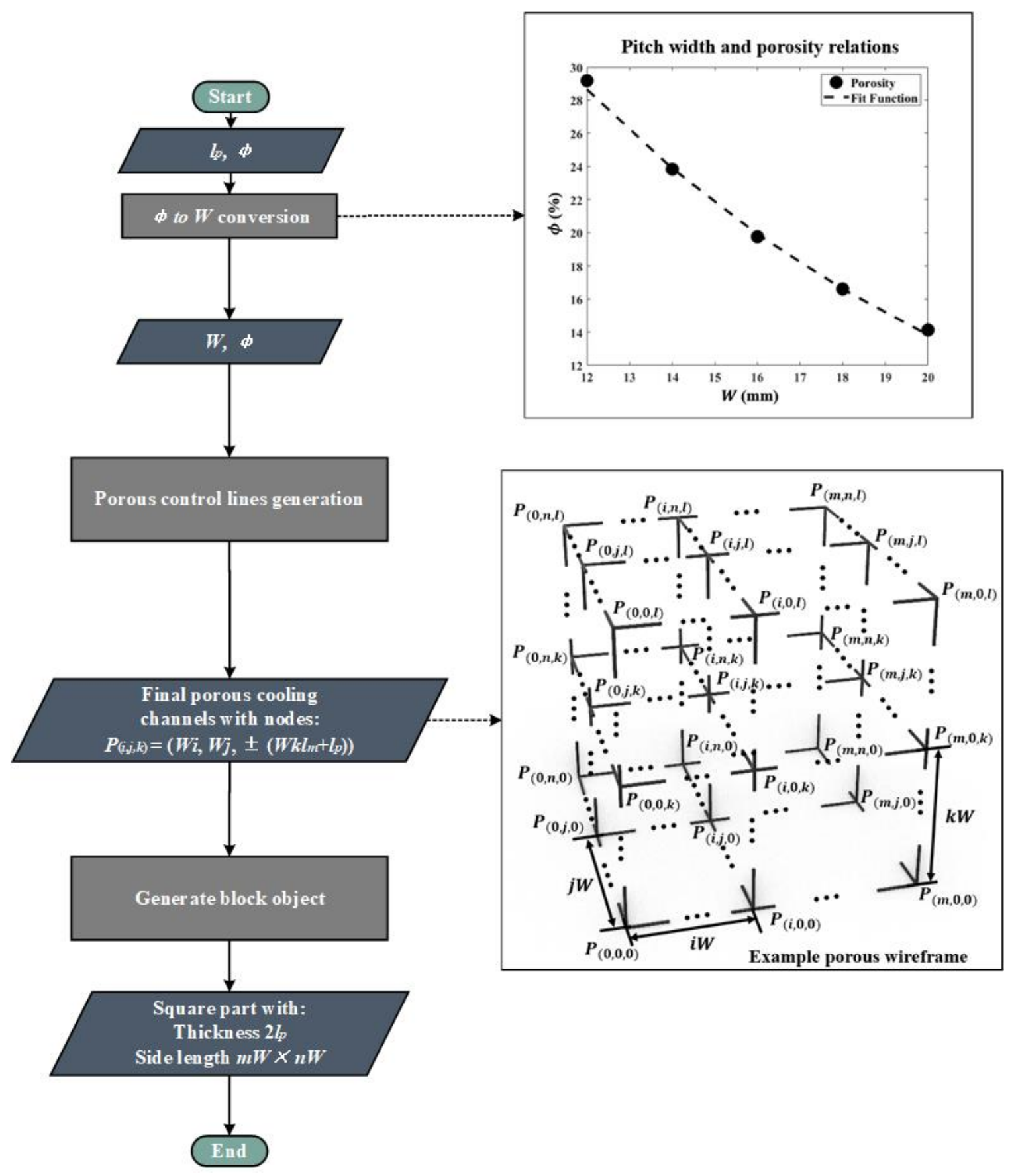

Figure 4-10. The porous cooling channels generation method 
For the porous cooling channels, the design parameters are $l_{p}$ and $\phi$. To generate the cooling channel control lines, relation between the pitch width of the porous cooling channels $W$ and the porosity $\phi$ is derived in Figure 4-10, where the fitting function is provided in equation (4-3):

$$
\phi=85.4 e^{-0.1 W}
$$

As shown in Figure 4-10, a convertion from $\phi$ to $W$ is performed at the beginning of the porous structure generation process. Different from the CPS generation algorithm, the spacing between the cooling plane is directly set to the cooling channel cell size of $W$ so that a uniform $\phi$ can be found at different mold positions. Hence, the wireframe of the porous structures is directly generated with the node coordinate calculation derived in equation (4-4):

$$
P_{(i, j, k)}=\left(i W, j W, \pm\left(k W+l_{m}+l_{p}\right)\right)
$$

where $P_{(i, j, k)}$ represents the node at the intersection among $i^{\text {th }}$ row, $j^{\text {th }}$ column, and $k^{\text {th }}$ cooling plane, and the \pm sign indicates wether a given node of porous cooling structures is above (+) or below (-) the part.

Based on the CPS and porous generation method provided in Figure 4-9 and Figure 4-10, the simulation files for all combinations of the design parameters can be generated as shown in Figure 4-11. For CPS cooling channels, the program updates $l_{m}(i), S(j)$, and $l_{p}(k)$ values to generate the IGES CPS cooling channel files named $l_{m}(i)_{-} S(j)_{-} l_{p}(k)$.igs, and the STL part file named $S(j) l_{l} l_{p}(k)$.stl. For the simulation files generation of the porous structure, the program iterates over all combinations of $\phi(i)$ and $l_{p}(j)$ values, and provides the IGES porous cooling channel files named $\phi(i) \_l_{p}(j)$.igs, and the STL part files named $\phi(i)_{-} l_{p}(j)$.stl. As discussed in Section 3.1.2, the $h_{1}$ and $h_{2}$ are not effective in terms of influencing the resulting part temepratures, 
(a) CPS simulation files generation algorithm

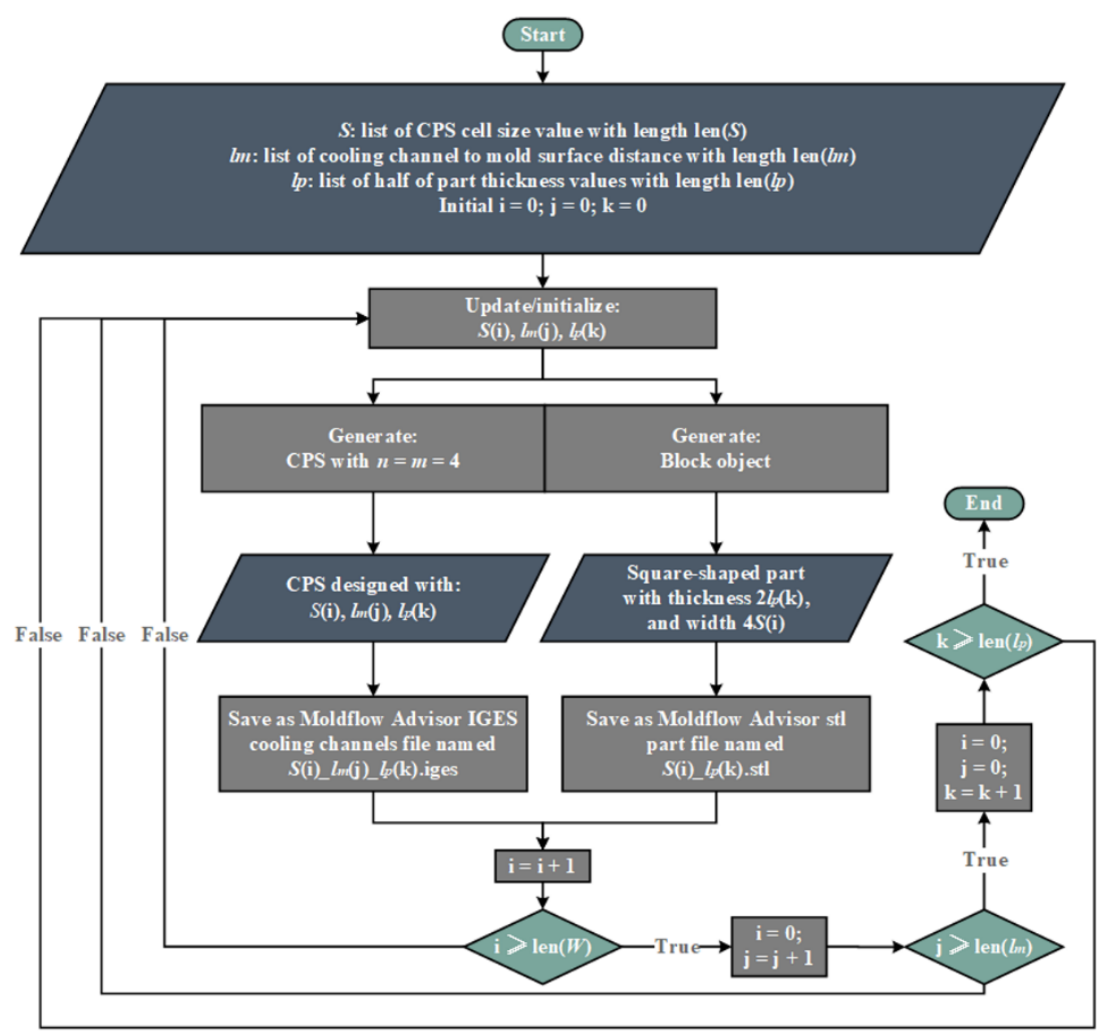

(b) Porous structure simulation files generation algorithm

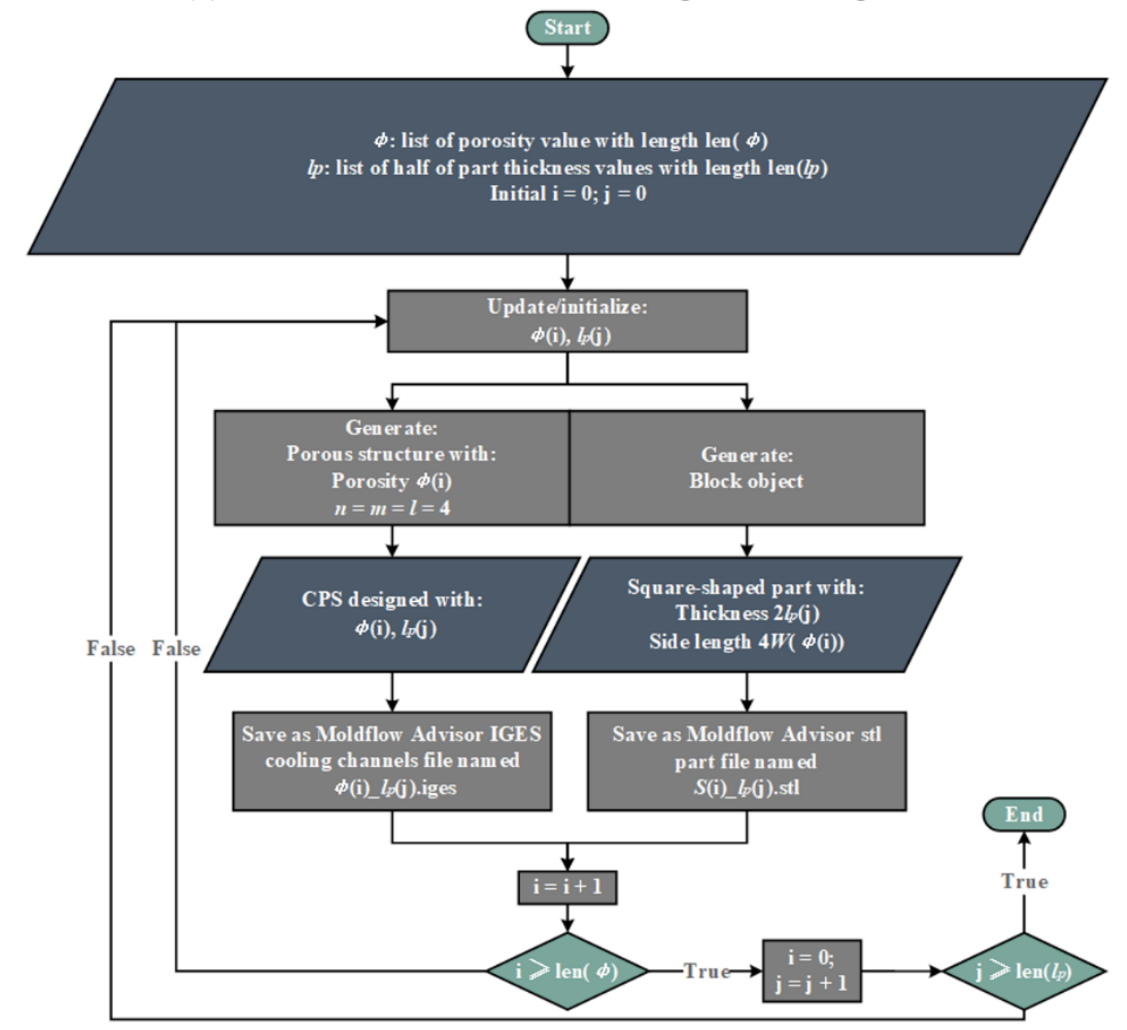

Figure 4-11. The CPS and porous structure simulation files generation algorithm 
therefore constant value to set to $h_{1}$ and $h_{2}$ as $12 \mathrm{~mm}$, which is the minimum value of the pitch width. Thus, the parameter $h_{1}$ and $h_{2}$ are set as $12 \mathrm{~mm}$ during the cooling channels and part generation process of the CPS and porous structure. In addition, the number of cooling cells of the CPS and porous structure is fixed as $n=m=4$, and $n=m=l=4$, respectively, to achieve a stable flow rate at the center of the cooling planes that is closest to the part surface without causing a significant increase of the simulation time. Note that the cooling channel to mold surface distance of the porous structure is not considered as a design parameter for porous structure. This distance is set as half of the pitch width values $W(\phi(i))$, since a invariant porosity over the mold is desired to ensure the accuracy of the training data. The generated simulation files are then imported into the Moldflow Advisor ${ }^{\circledR}$ simulation as shown in Figure 3-4a and Figure 4-12a. The input parameters for the simulations are provided in Table 4-1. Note that the the inlet flow rate of the CPS and porous cooling simulations is different from which of the zigzag and spiral cooling simulations. The flow rate for CPS and porous structures is set to $20 \mathrm{lit} / \mathrm{min}$, since it is the inlet

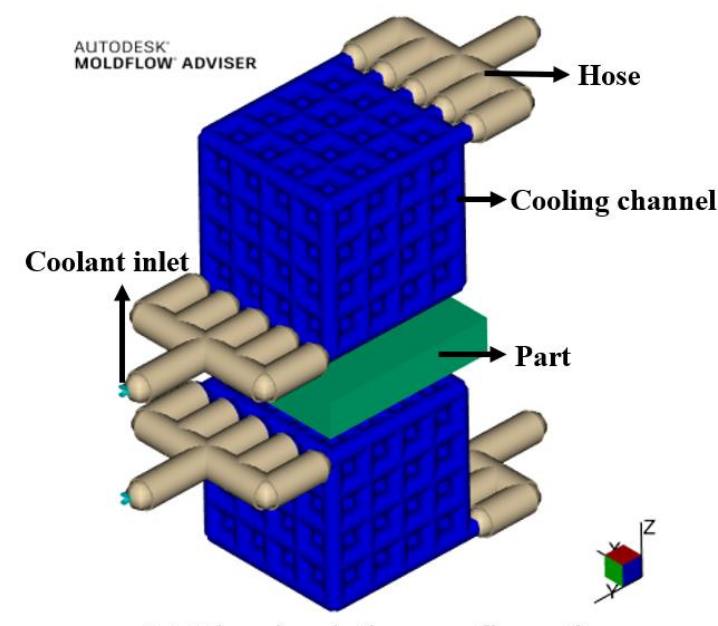

(a) The simulation configuration for porous training data collection

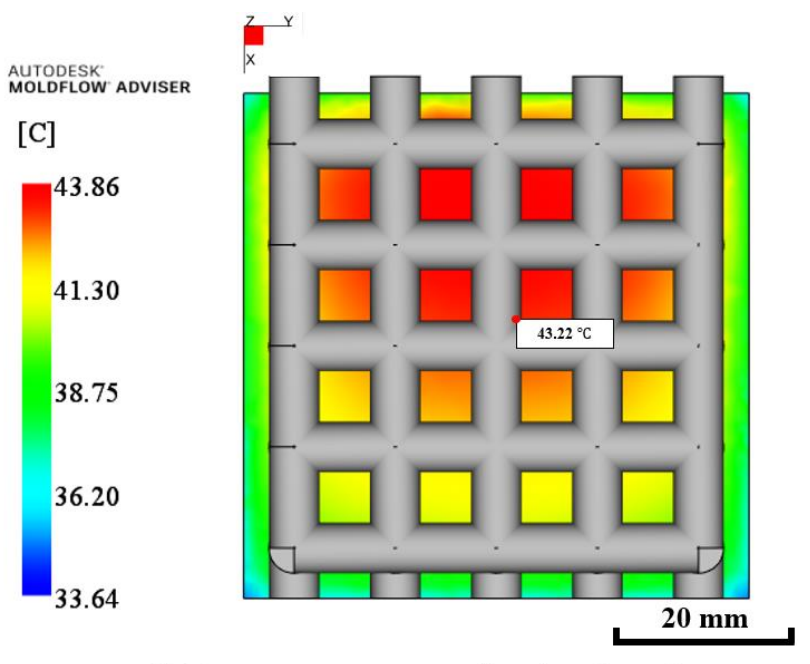

(b) Part temperature collection location

Figure 4-12. Example simulation configuration and temperature distribution result for porous cooling channels 
flow rate that makes the major portions of the cooling channels exhibit a flow rate that is close to 5 lit/min, which is the same as coolant flow rate for zigzag and spiral cooling channels. This inlet flow rate allows the comparison between different topological designs of the cooling channels together on different regions of the same part. Example simulation results are provided in Figure $3-4 \mathrm{~b}$ and Figure $4-12 \mathrm{~b}$. The temperature data is collected at the center of the part surface, since the flow rate of CPS and porous cooling channels are not stable at the positions closer to the inlets and outlets.

\subsection{Supervised machine learning process}

In this study, a supervised machine learning process is applied to train the surrogate temperature prediction model for different cooling channel topologies including zigzag, spiral, porous, and CPS. There are mainly two reasons of deriving a machine learning surrogate model for temperature prediction. First, the theoretical models such as the temperature model proposed by $\mathrm{Xu}$ et al. [10] cannot be generally adapted and applied to different novel designs of conformal cooling channel geometries due to their various types of design parameters. Even if the basic heat transfer theory in Section 1.2 can be modified for novel conformal cooling designs such as CPS [13], the accuracy of the adapted model cannot be guaranteed. However, the surrogate model proposed in this thesis can be effectively tested by the testing data, and the loss functions during the training process. In addition, the computational cost of the temperature prediction is significantly decreased, where the approximate computing time between the Moldflow Advisor ${ }^{\circledR}$ and the surrogate temperature model for different cooling channel topologies is compared on a computer with NVIDIA ${ }^{\circledR}$ GEFORCE ${ }^{\circledR}$ GTX graphics card, intel ${ }^{\circledR}$ i7-8750H CPU, and 16 GB RAM. The comparison results as shown in Table 4-2 validates that the surrogate temperature model provided in this work is more efficient in predicting local part temperature during the optimization 
process. The detailed explanation of the ANN training process is given in Section 4.2.1, where the evaluation result of the finally trained model is provided in Section 4.2.2.

Table 4-2. Approximate computational cost of the simulation software and the machine learning temperature prediction model

\begin{tabular}{|l|l|l|}
\hline $\begin{array}{l}\text { Types of the cooling channel } \\
\text { topology }\end{array}$ & $\begin{array}{l}\text { Moldflow Advisor cooling } \\
\text { simulation }\end{array}$ & $\begin{array}{l}\text { Machine learning temperature } \\
\text { prediction model }\end{array}$ \\
\cline { 1 - 2 } Spiral & $30 \sim 60$ minutes & \\
\hline Zigzag & $1 \sim 5$ minutes & \multirow{2}{*}{0.5 seconds } \\
\hline Porous & $5 \sim 10$ minutes & \\
\hline CPS & $5 \sim 10$ minutes & \\
\hline
\end{tabular}

\subsubsection{ANN training process}

The ANN is selected in this work to predict the heat transfer performance of the conformal cooling channels and build the surrogate model. ANN is a massively parallel distributed processor that consists of simple processing units. There are two reasons that ANN is chosen in this study. First, ANN has the computing power to solve complex problems that are currently intractable due to its large scale of parallel distributed structure as well as the ability to learn and generalize [47]. Second, the accuracy of ANN can be continuously improved by importing additional training data without changing its original architecture. Among different types of supervised machine learning method, it should also be noted that the ANN is a type of these strategies selected in this work to validate the proposed MLACCD method, while the proposed method is not limited to be achieved through ANN. The layout of the ANN is provided in Figure 4-13, where it has two hidden layers, and the input layer is not the same for different topologies of the cooling channels. For instance, the input layer for CPS is in the form of $\left[S, l_{m}, l_{p}\right]$, which is different from $\left[\phi, l_{p}\right]$ for porous structures, and $\left[W, l_{m}, l_{p}\right]$ for zigzag and spiral cooling channels due to their different identified design parameters. There are two hidden layers in the ANN, where each hidden layer contains 20 


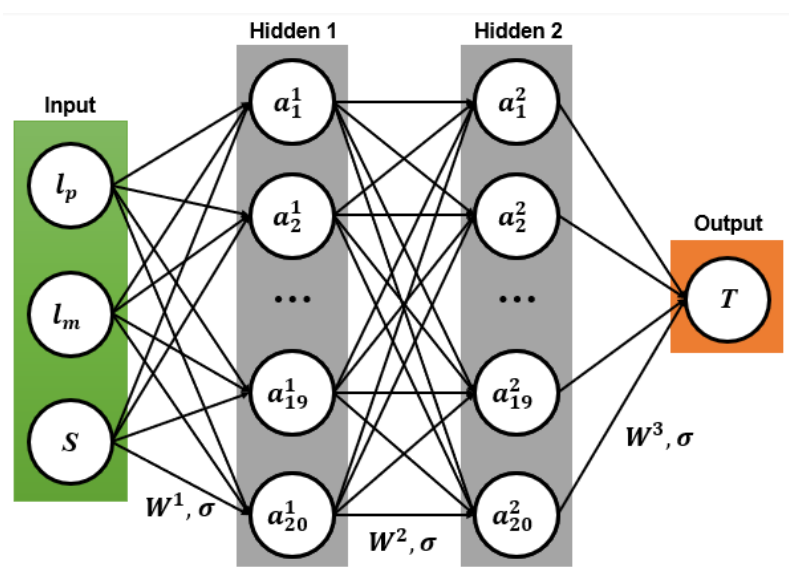

(a) ANN layout for CPS

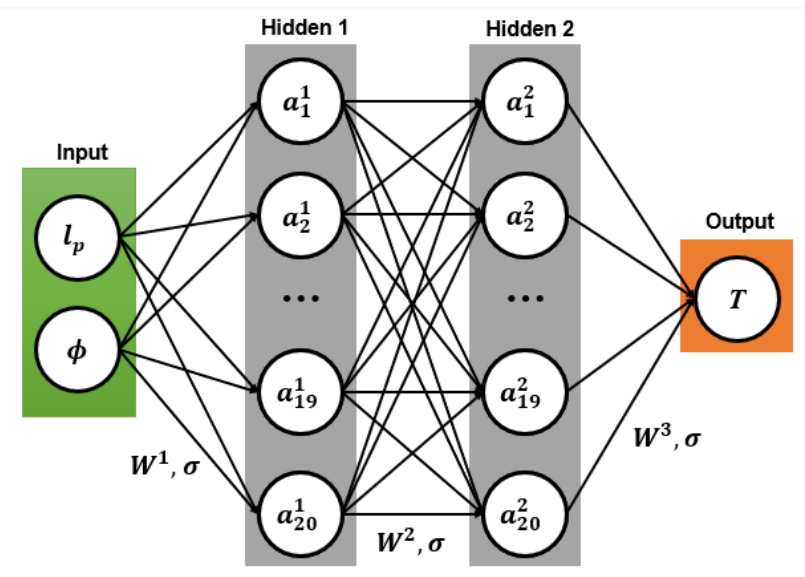

(b) ANN layout for porous structures

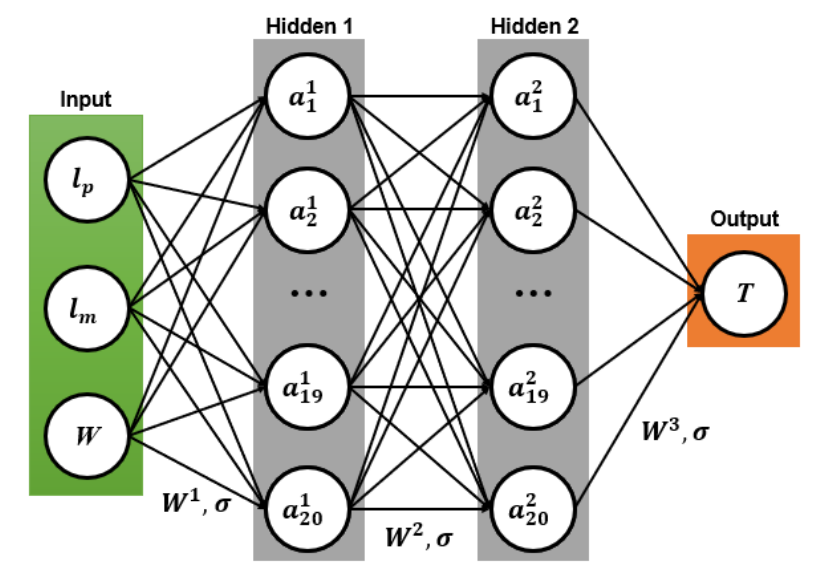

(c) ANN layout for zigzag and spiral cooling channels

Figure 4-13. The ANN layout for different cooling channel topologies

nodes. To pass the information between layers, the propagation function is used to calculate the weighted sum of the node outputs as shown in equation (4-5):

$$
z_{j}^{i}=\sum_{k=1}^{n} W_{j k}^{i} a_{k}^{i-1}+b_{j}^{i}
$$

where $z_{j}^{i}$ is the input value for $j^{t h}$ neuron of the $i^{t h}$ layer, $a_{k}^{i-1}$ is the output from $k^{t h}$ neuron of the $i-1^{\text {th }}$ layer, $W_{j k}^{i}$ represents the weight of the $a_{k}^{i-1}$ on $z_{j}^{i}, n$ represents the number of neurons in $i-1^{\text {th }}$ layer, and $b_{j}^{i}$ is the bias function between $i-1^{\text {th }}$ and $i^{\text {th }}$ layer. To non-linearize the input value of each neuron, an activation function is used as shown in equation (4-6): 


$$
a_{j}^{i}=\sigma\left(z_{j}^{i}\right)
$$

where $\sigma$ is the Sigmoid activation function, $a_{j}^{i}$ is the node output value. During the training process, the weight of each neuron is adjusted to obtain the trained machine learning surrogate model with a learning rate of 0.005 and a 30000 number of training epochs. The performance of this ANN configuration is evaluated by the loss function as shown in Figure 4-14, where the ANN structure (i.e. two hidden layers, 20 neurons in each layer, etc.) and the training parameters (i.e. learning rate of $0.005,30000$ training epochs, etc.) are selected to achieve a steady decrease of the loss function during the training process.

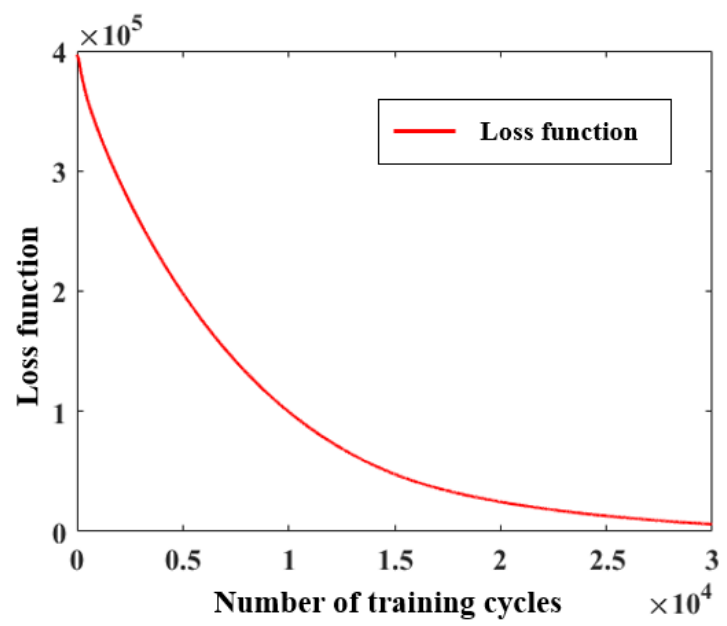

Figure 4-14. Loss function of the machine learning process

As discussed in Section 4.1, the training data is generated and collected from the Moldflow Advisor $^{\circledR}$ simulation. In total, more than 1000 sets of the training data are built for the zigzag, spiral, porous, and CPS cooling systems as shown in Figure 4-15a to Figure 4-15c, while $20 \%$ of them are randomly chosen as the test data. The amount of the training data is determined based on the accuracy of the model. Initially, a small number of the training data sets is created and fed into the ANN, and a large error between the predicted temperature and the test data exists. To 
improve the accuracy of the model, an increasing amount of the training data is fed into ANN until the accuracy of the model is precise enough for the optimization process. The number of the training data sets reported in this work is the final amount of the training data that is able to successfully train the surrogate temperature model with a reasonable model accuracy. In some ranges of the parameters, the rate of temperature variation is higher, so more underlying patterns of data need to be studied by ANN in these ranges. Therefore, specifically importing these training data will improve the accuracy of the temperature prediction model faster. For zigzag and spiral

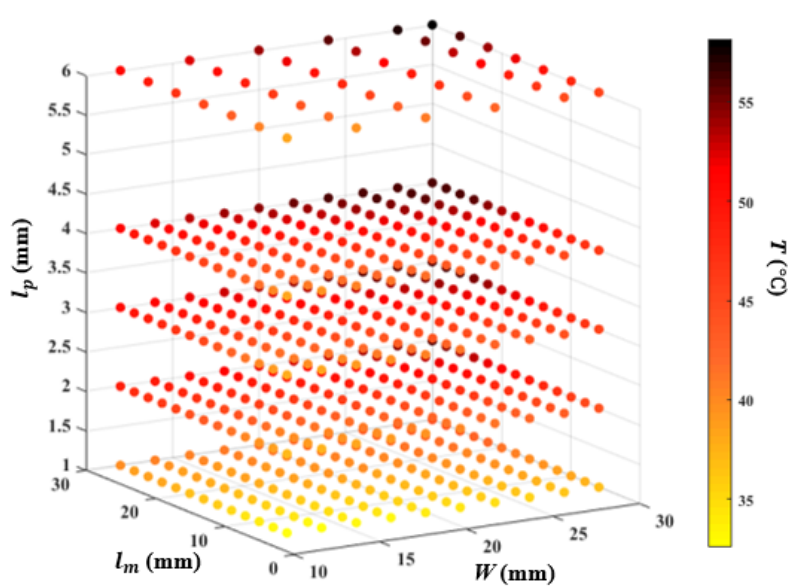

(a) Training data for spiral and zig-zag cooling channels

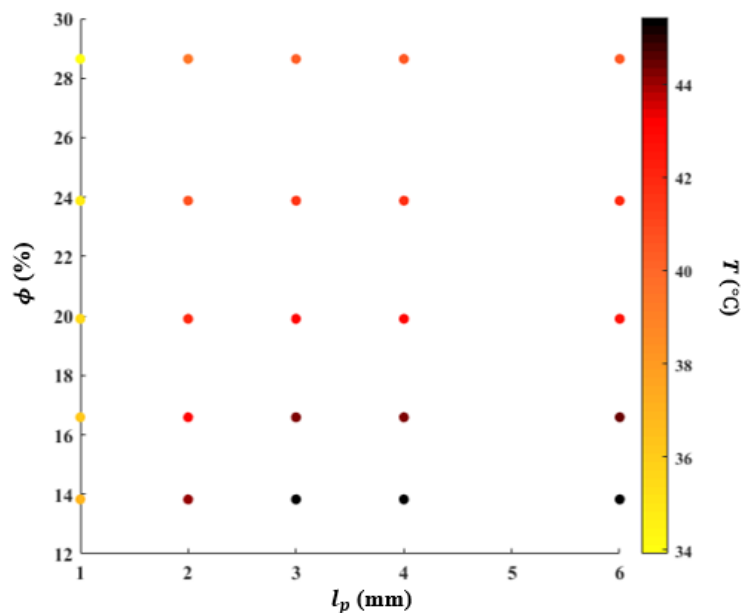

(b) Training data for porous cooling channels

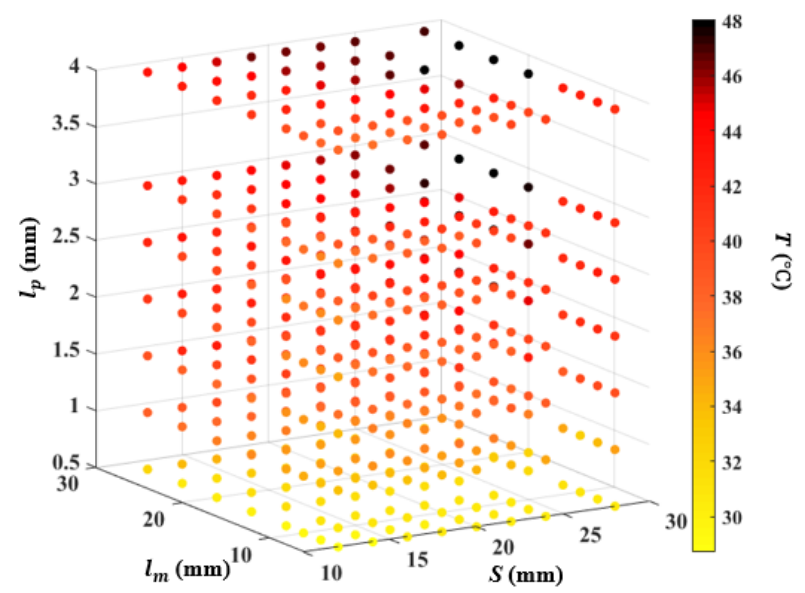

(c) Training data for CPS

Figure 4-15. Training data for the supervised machine learning process 
cooling channels, more test data is selected from the range of $l_{p} \leq 4 \mathrm{~mm}$. For CPS, more test data is generated for the ranges of $l_{p} \leq 4 \mathrm{~mm}, l_{m} \leq 12 \mathrm{~mm}$ and $S \leq 20 \mathrm{~mm}$. The final trained surrogate temperature prediction model is provided in Figure 4-16, where the evaluation results (i.e. computation time, temperature prediction error) of this model is provided in Section 4.2.2.

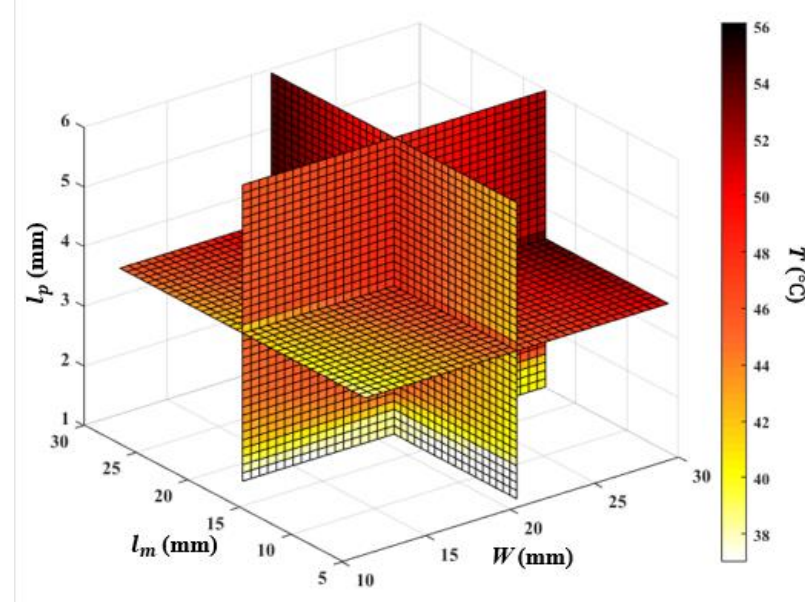

(a) Part surface temperature prediction for spiral and zig-zag cooling channels

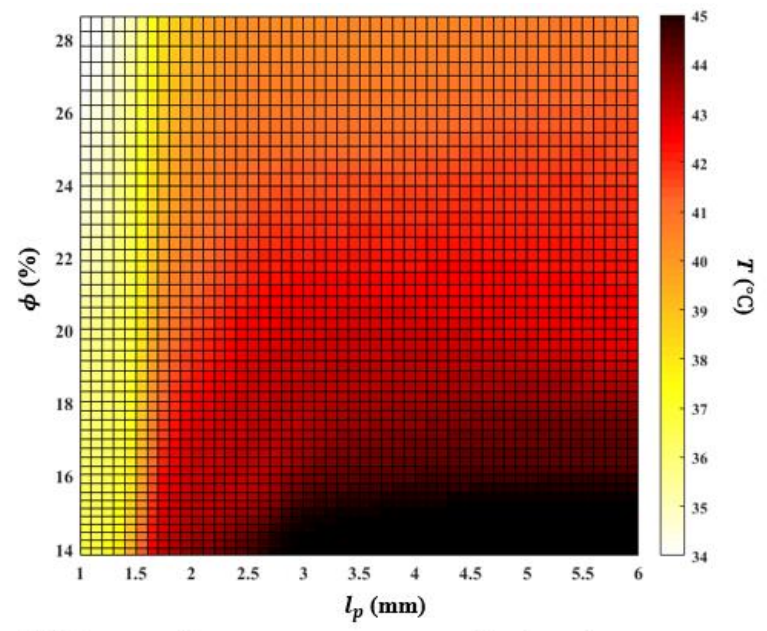

(b) Part surface temperature prediction for porous cooling channels

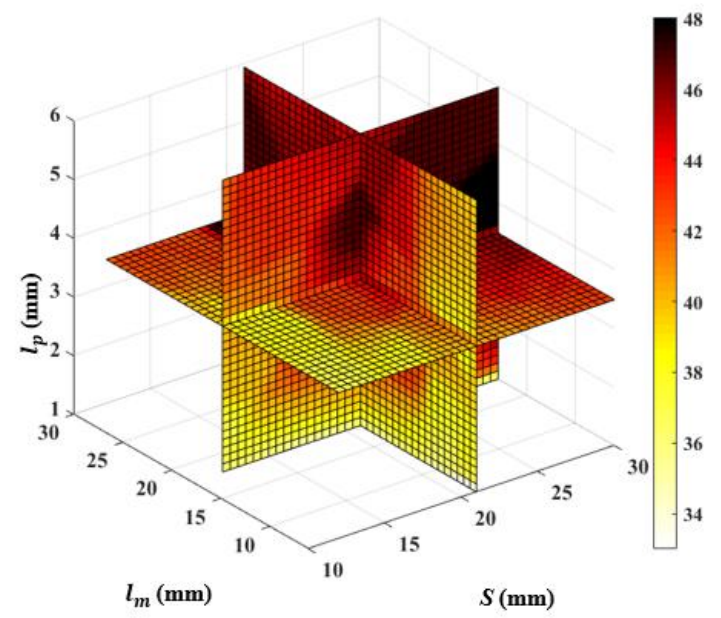

(c) Part surface temperature prediction for CPS cooling system

Figure 4-16. Part temperature prediction from the machine learning surrogate model

\subsubsection{Evaluation of the surrogate temperature model}

In this section, the evaluation results of the trained surrogate temeprature model is provided. There are two types of evaluations performed for every training attempt. First, the loss function is 
calculated during each training process, which reflects the quality of the training algorithm. The loss function is calculated based on the mean squared error formula as shown in equation (4-7):

$$
\operatorname{MSE}=\frac{1}{N} \sum_{i=1}^{N}\left(y_{i}-\widehat{y}_{l}\right)^{2}
$$

where MSE is the mean squared error, $N$ is the size of the sample, $\widehat{y}_{\iota}$ represents the predicted values, and $y_{i}$ is the true value. After each training process, the parameters of the ANN are adjusted if the loss function does not exhibit a steady decrease during that training. The loss function for the finalized ANN with 30000 training cycles and a learning rate of 0.005 is shown in Figure 4-14, where a steady decrease is observed. Second, the accuracy of the trained temperature prediction model is evaluated based on the maximum temperature difference between the test data and the prediction from the surrogate model. The test results are provided in Table 4-3, the maximum temperature difference for porous, spiral, zigzag, and CPS are all below $0.5^{\circ} \mathrm{C}$, which indicates that the surrogate temperature prediction model is accurate since it has reached the default temperature resolution of the MLACCD software (within the TVM process of the software, the design parameter update process stops once the predicted temperature converges, or the difference between predicted temperature and target temperature has reached $\pm 0.5^{\circ} \mathrm{C}$ ).

Table 4-3. The accuracy of the surrogate temperature prediction model

\begin{tabular}{|l|l|l|l|}
\hline Topology & Porous & Spiral and zigzag & CPS \\
\hline $\begin{array}{l}\text { Maximum temperature difference between the } \\
\text { prediction and test data }\left({ }^{\circ} \mathrm{C}\right)\end{array}$ & 0.23 & 0.28 & 0.47 \\
\hline Test data percentage $(\%)$ & 20 & & \\
\hline
\end{tabular}

\subsection{Summary}

To achieve a fast and accurate prediction of the part temperature based on the given conformal cooling design parameters, the supervised machine learning is performed with two steps: (1) construction of the training data; (2) training the machine learning surrogate model through ANN. 
Due to a large amount of the required training data, algorithms are designed to automatically construct the IGES cooling channel files and their corresponding part files. These simulation files are imported into the Moldflow Advisor ${ }^{\circledR}$ simulation software to gather the temperature data. The training data is then constructed by combining the design parameter sets with their corresponding resulting temperature of the part surface. During the training process, over 1000 sets of the training data are imported into an ANN with two hidden layers, where the design parameters of the cooling channels are set as the input layer, and the resulting temperature are set as the output layer. The loss function during the training presents a steady decrease, and the maximum model error is less than $0.5^{\circ} \mathrm{C}$. As a result, a surrogate model that is able to achieve a fast and accurate prediction of the part surface temperature is trained. The derived machine learning surrogate temperature prediction model is then applied in Chapter 5 to aid the rapid temperature prediction during the TVM process. 


\section{CHAPTER 5}

\section{Temperature variance minimization process}

In this chapter, different optimization strategies are discussed for spiral, zigzag, porous, and CPS cooling topologies in Sections 5.1 to 5.4 based on the surrogate temperature prediction model. The differences between the optmization strategies of these cooling topologies are caused by the large variation among the geometrical designs of their cooling systems. For each cooling topology, the design parameters of the cooling channels are optimized to minimize the part temperature variance based on the given part thickness distributions. Specifically for the cooling topologies where the pressure drop is high such as zigzag and spiral, sequential optimizations are used to obtain a cooling channel which can reduce the temperature variance with the shortest length. The optimal cooling channel control points are generated based on these optimized design parameters, and the final machine learning aided conformal cooling channels are generated by connecting these control points.

\subsection{Optimization of spiral cooling channels}

The general optimization procedure for spiral cooling channels includes four steps: generation of control lines, generation of spiral W-optimized control points (WOCP), spiralization of WOCP, and $l_{m}$ adjustment of spiral WOCP as shown in Figure 5-1. For a general example of spiral design area with multiple thickness maxima as shown in Figure 5-2, the area division based on the thickness contour is first applied to divide it into multiple spiral design areas with single thickness maxima. Specifically, the division line is selected as boundary thickness contours of those design areas with distinct local maxima points. For a derived spiral cooling surface with single local maxima as shown in Figure 5-3, the control lines are first found to restrict the optimization space for the control points of the cooling channels. To derive the control lines, the point $p_{0}$ with maximum divergence is defined in equation (5-1): 


$$
\vec{\nabla}^{2} l_{p}\left(x_{p_{0}}, y_{p_{0}}, z_{p_{0}}\right)=\operatorname{MAX}\left(\vec{\nabla}^{2} l_{p}\left(x_{p_{A}}, y_{p_{A}}, z_{p_{A}}\right)\right)
$$

where $\left(x_{p_{0}}, y_{p_{0}}, z_{p_{0}}\right)$ is the coordinate of $p_{0}$, and $\left(x_{p_{A}}, y_{p_{A}}, z_{p_{A}}\right)$ is the coordinate of an arbitrary point $p_{A}$ on the example spiral cooling surface.

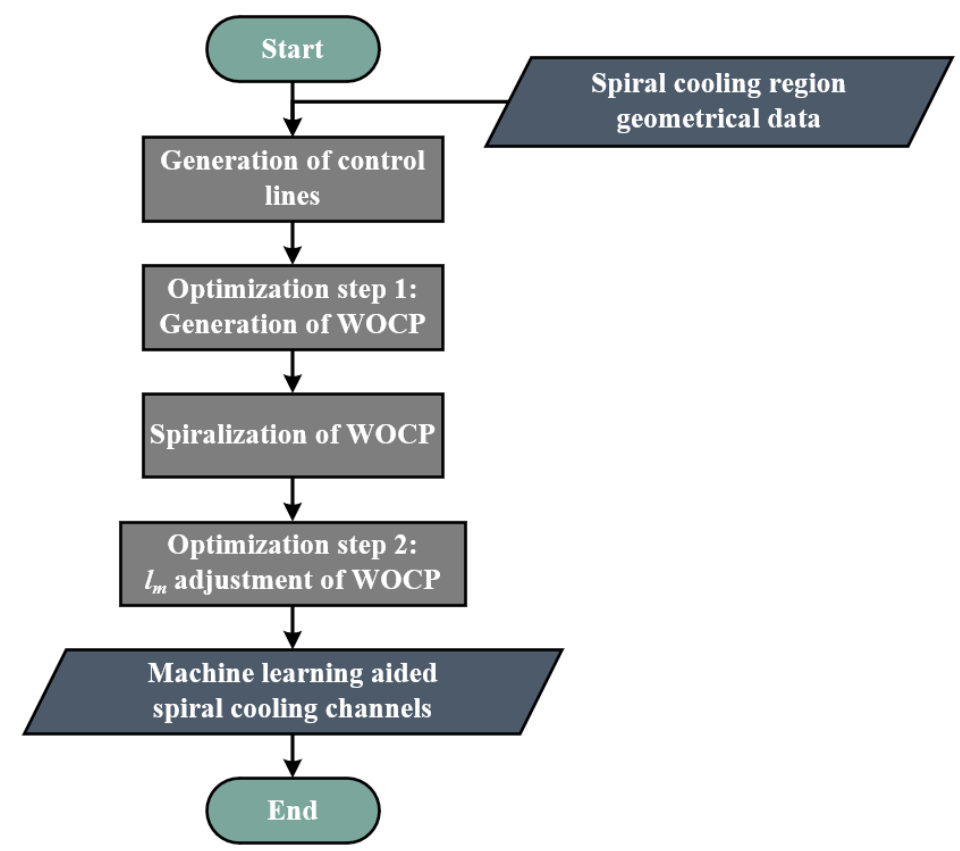

Figure 5-1. The design procedure of machine learning aided spiral cooling channels

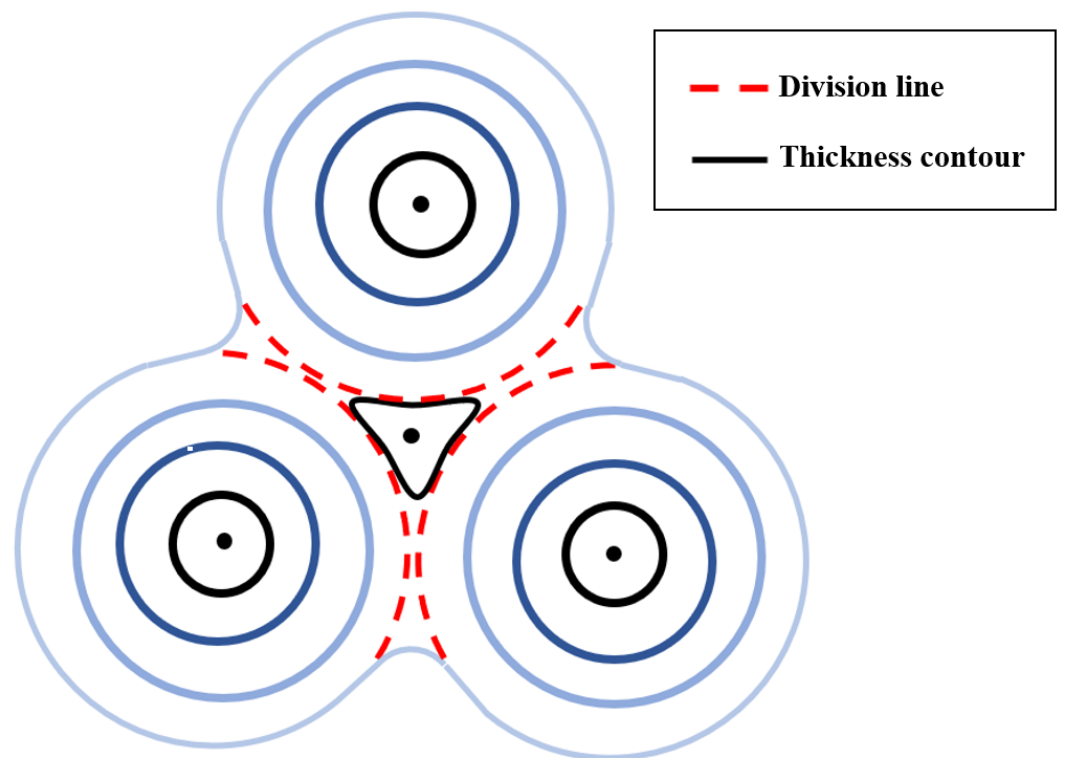

Figure 5-2. Division of spiral design areas from multiple local thickness maxima 
As shown in Figure 5-4, each control line $l_{i}$ is created so that it starts from $p_{0}$ with the direction of $\vec{\nabla} l_{p i}$ separated by an resolution angle $\Delta \theta$. The value of the resolution angle is related to the variation of $\vec{\nabla} l_{p i}$ on its tangent direction. For instance, suppose the value of $\vec{\nabla} l_{p i}$ is distributed along the part surface in an approximately isometric manner, the resolution angle $\Delta \theta$ can be set to a larger value. However, if the value of $\vec{\nabla} l_{p i}$ is varying along its tangent direction as shown in the

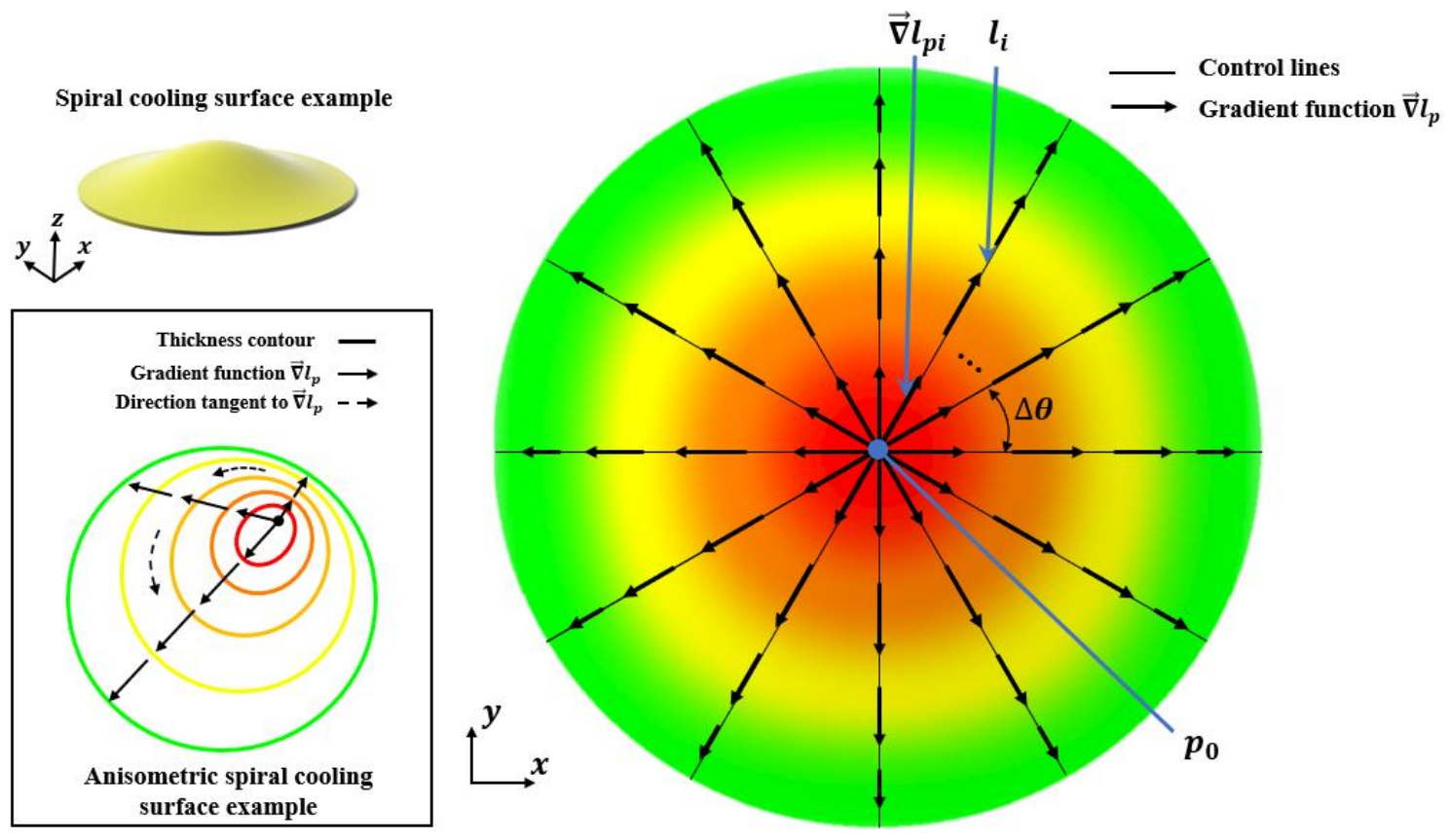

Figure 5-3. An example of a spiral cooling surface with gradient function and control lines

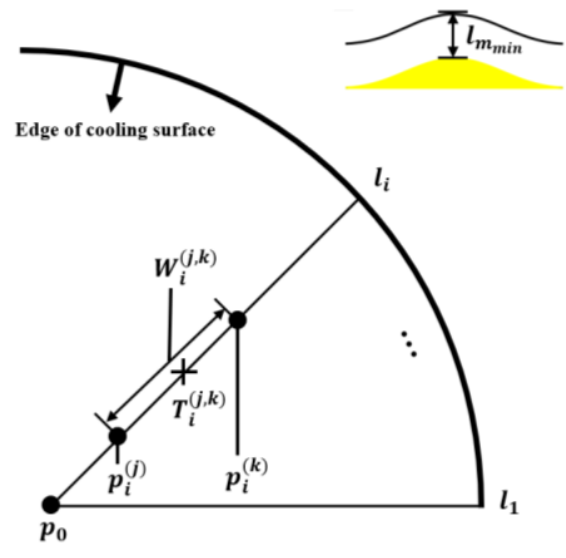

(a) 2-point system on control line

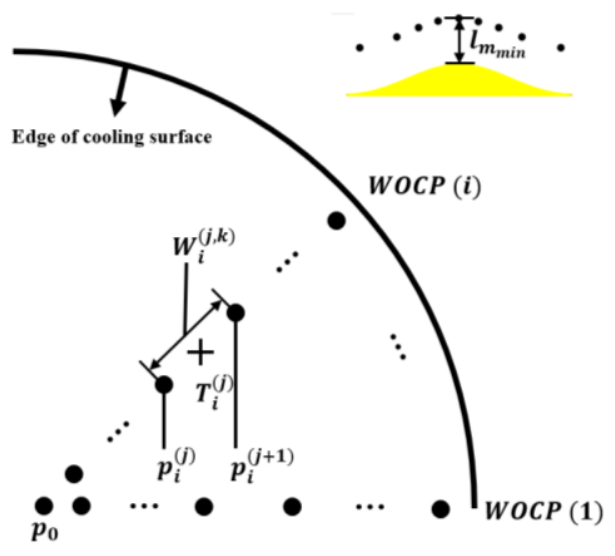

(b) 2-point system of $W O C P$

Figure 5-4. Generation of WOCP on a spiral cooling surface 
caption of Figure 5-3, a smaller value of $\Delta \theta$ is required since different designs of cooling channel control points need to be derived, which will be discussed in detail in the following paragraphs.

Second, the $W O C P$ for the spiral cooling channels for the example cooling surface is generated as shown in Figure 5-4. To create WOCP, the control lines are first lofted from the part surface by the minimum pitch to mold distance $l_{m_{\min }}=6 \mathrm{~mm}$. For each control line, the 2-point system $\left(p_{i}^{(j)}, p_{i}^{(k)}\right)$ for arbitrary points $p_{i}^{(j)}$ and $p_{i}^{(k)}$, and the corresponding temperature $T_{i}^{(j, k)}$ between $p_{i}^{(j)}$ and $p_{i}^{(k)}$ are defined in equations (5-2) and (5-3):

$$
\begin{gathered}
\left\{\left(p_{i}^{(j)}, p_{i}^{(k)}\right) \mid p_{i}^{(j)}, p_{i}^{(k)} \in L(i)\right\} \\
T_{i}^{(j, k)}=S M L T M_{\text {spiral }}\left(\operatorname{AVG}\left(l_{p i}^{(j)}, l_{p i}^{(k)}\right), W_{i}^{(j, k)}, \operatorname{AVG}\left(l_{m i}^{(j)}, l_{m i}^{(k)}\right)\right)
\end{gathered}
$$

where $L(i)$ represents the set of the points on control line $l_{i}, S M L T M_{\text {spiral }}$ is the surrogate model relating the design parameters and resulting part surface temperature for spiral cooling channels, $l_{p i}^{(j)}$ indicates the half of part thickness measured from the cooling surface geometry below $p_{i}^{(j)}, l_{m i}^{(j)}$ represents the distance from $p_{i}^{(j)}$ to the cooling surface, $W_{i}^{(j, k)}$ is the distance between $p_{i}^{(j)}$ and $p_{i}^{(k)}, T_{i}^{(j, k)}$ is the resulting surface temperature of a part with $l_{p}=A V G\left(l_{p i}^{(j)}, l_{p i}^{(k)}\right)$ cooled by spiral cooling channels with $W=W_{i}^{(j, k)}, l_{m}=A V G\left(l_{m i}^{(j)}, l_{m i}^{(k)}\right)$. From the set of points on each control line $l_{i}$, a sub-point set $W O C P(i)$ is found through the spiral optimization step 1 as expressed in Table 5-1. During the optimization iteration, the quantity $m$ and the coordinates of the control points $p_{i}^{(j)}$ are updated so that the resulting part surface temperature $T_{i}^{(j, j+1)}$ predicted by SMLTM $M_{\text {spiral }}$ is the closest to the target temperature $T_{g}$ for each $\left(p_{i}^{(j)}, p_{i}^{(j+1)}\right)$. 
Table 5-1. The temperature variance minimization (TVM) strategies for each cooling topology

\begin{tabular}{|c|c|c|}
\hline Topology & TVM strategy & \multirow{3}{*}{$\begin{array}{l}\text { Step 2: } \\
\text { To Find: } \Delta h_{i}^{(j)}, \mathrm{j}=1,2, \ldots, m \\
\text { Minimize: }\left|T_{g}-T_{i}^{(j, j+1)}\right|, \mathrm{j}=1,2, \ldots, m-1 \\
\text { S.T.: } 12 \mathrm{~mm} \leq W_{i}^{(j, j+1)} \leq 30 \mathrm{~mm} \\
\quad 0 \mathrm{~mm} \leq \Delta h_{i}^{(j)} \leq 24 \mathrm{~mm}\end{array}$} \\
\hline Spiral & $\begin{array}{l}\text { Step 1: } \\
\text { To Find: } m, p_{i}^{(j)}, \mathrm{j}=1,2, \ldots, m \\
\text { Minimize: }\left|T_{g}-T_{i}^{(j, j+1)}\right|, \mathrm{j}=1,2, \ldots, m-1 \\
\text { S.T.: } p_{i}^{(j)} \in L(i) \\
\quad 12 \mathrm{~mm} \leq W_{i}^{(j, j+1)} \leq 30 \mathrm{~mm} \\
\quad l_{m i}^{j}=l_{m_{\min }} \\
\quad m \geq 2\end{array}$ & \\
\hline Zigzag & $\begin{array}{l}\text { Step 1: } \\
\text { To Find: } m, p_{1 \sim n}^{(j)}, \mathrm{j}=1,2, \ldots, m \\
\text { Minimize: }\left|T_{g}-T^{(j, j+1)}\right|, \mathrm{j}=1,2, \ldots, m-1 \\
\text { S.T.: } p_{i}^{(j)} \in L(i) \\
\quad 12 \mathrm{~mm} \leq W_{i}^{(j, j+1)} \leq 30 \mathrm{~mm} \\
\quad l_{m i}^{j}=l_{m_{\min }} \\
\quad m \geq 2\end{array}$ & \\
\hline Porous & $\begin{array}{l}\text { To Find: } \phi_{i} \\
\text { Minimize: } \Delta T_{i} \\
\text { S.T.: } 10 \% \leq \phi_{i} \leq 30 \%\end{array}$ & \\
\hline CPS & $\begin{array}{l}\text { For each } l_{p} \text { : } \\
\text { To Find: } S_{i}, l_{m j}, i, j=1,2, \ldots, n-1 \\
\text { Minimize: }\left|T_{g}-\mathrm{T}_{(i, j)}\right| \\
\text { S.T.: } 12 \mathrm{~mm} \leq S_{i} \leq 30 \mathrm{~mm} \\
\quad 6 \mathrm{~mm} \leq l_{m j} \leq 30 \mathrm{~mm}\end{array}$ & \\
\hline
\end{tabular}

Third, the spiralized WOCP is generated as shown in Figure 5-5 by applying the similar boundary distance mapping (BDM) method proposed by Wang et al. [14]. As shown in Figure $5-5$, there is an associated distance $d_{i}^{(j-1, j)}$ between $p_{i}^{(j)}$ and $p_{i}^{(j-1)}$ in the original WOCP $(i)$. To derive spiralized WOCP, each $p_{i}^{(j)}$ is moved towards $p_{0}$ by $\Delta d_{i}$ defined in equation (5-4):

$$
\Delta d_{i}=\frac{\Delta \theta}{2 \pi} d_{i}^{(j-1, j)} i
$$

where $\Delta d_{i}$ is the distance for point $p_{i}^{(j)}$ to be moved. 


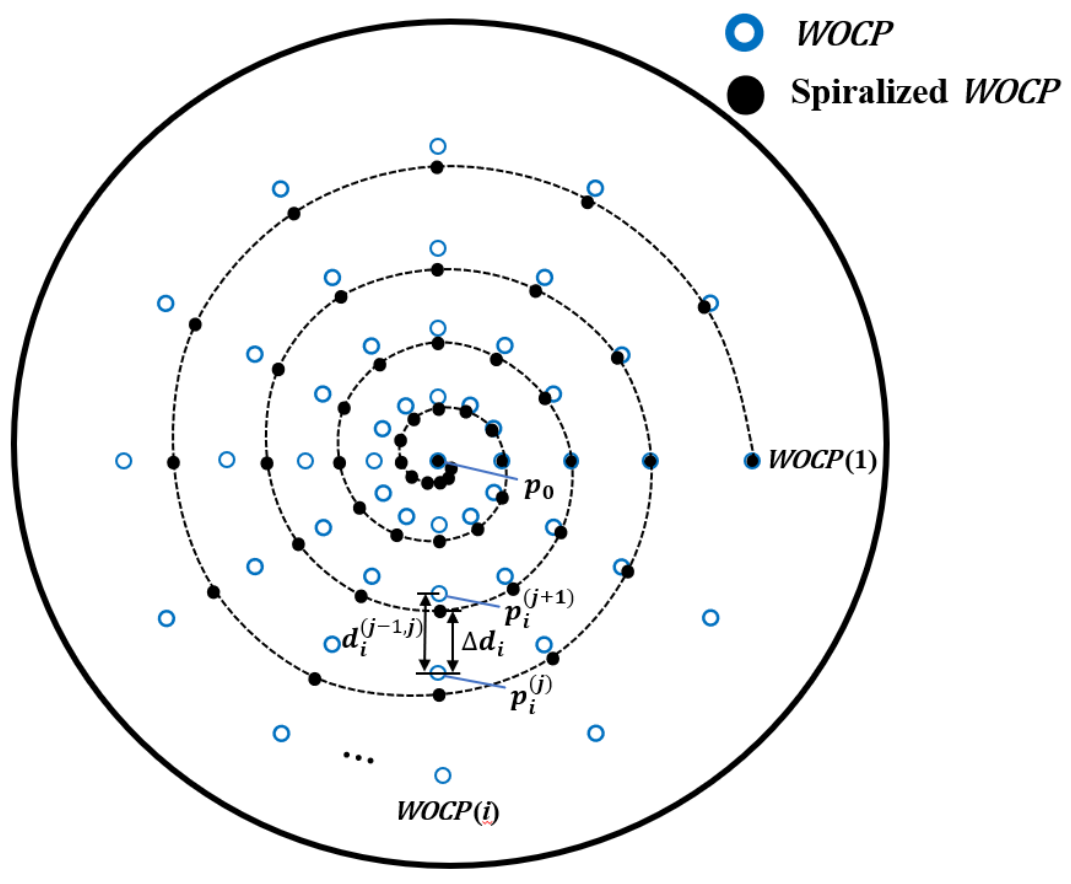

Figure 5-5. Spiralization of WOCP

Temperature variance minimized $W O C P(i)$

Spiralized $W O C P(i)$

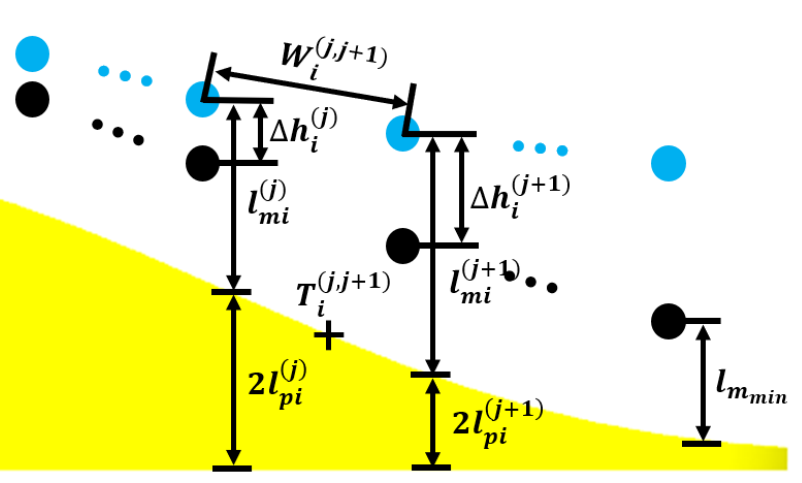

Figure 5-6. Generation of $l_{m}$ - optimized control points of spiral cooling channels

Finally, the $l_{m}$ values of each point in spiralized WOCP are adjusted as shown in Figure 5-6.

To illustrate, the adjustment of the pitch to mold surface distance $l_{m i}^{(j)}$ as shown in is defined in equation (5-5): 


$$
l_{m i}^{j}=l_{m_{\min }}+\Delta h_{i}^{j}
$$

where $\Delta h_{i}^{j}$ is the additional height added to the distance from $p_{i}^{(j)}$ to the cooling surface. During the spiral optimization step 2 as shown in Table $5-1, \Delta h_{i}^{(j)}$ is updated so that for each $\left(p_{i}^{(j)}, p_{i}^{(j+1)}\right)$, the objective function $\left|T_{g}-T_{i}^{(j, j+1)}\right|$ is minimized for $j=1,2, \ldots, m$. The value of $\Delta h_{i}^{(j)}$ is selected as $0.5 \mathrm{~mm}$ in this study, where a smaller $\Delta h_{i}^{(j)}$ value won't cause noticeable difference on the resulting temperature distribution based on our experience. During the optimization, the predicted part surface temperature converges to the target temperature with small variations, which indicates the completion of the optimization process. For the case that the predicted temperature is unable to reach the target temperature, adjustments can be made for the target temperature, and the range of the optimization parameters. At the end of the spiral optimization process, the adjusted control points are connected to form the machine learning aided spiral cooling channels as shown in Figure 5-7.

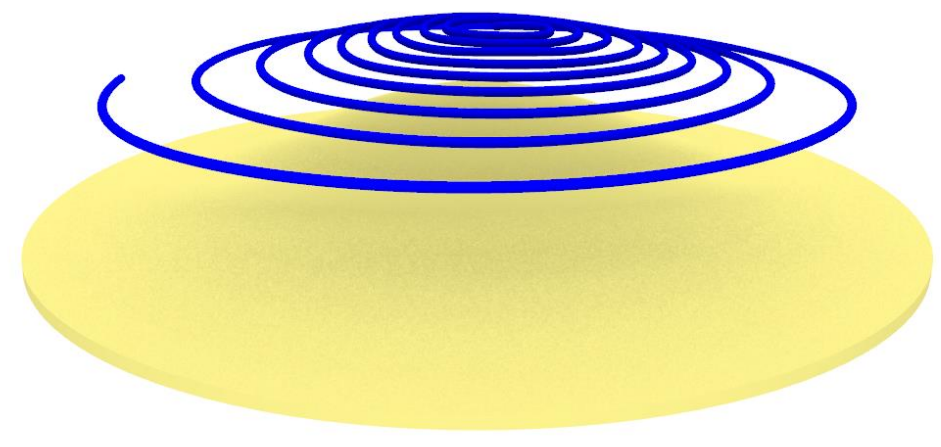

Figure 5-7. Machine learning aided spiral cooling channel 


\subsection{Optimization of zigzag cooling channels}

As shown in Figure 5-8, the optimization process of a zigzag conformal cooling channels includes three steps: generation of control lines, generation of zigzag $W O C P$, and $l_{m}$ adjustment of zigzag WOCP. To design the cooling channels with the highest cooling controllability, the control lines are created along the main direction of $\vec{\nabla} l_{p}$ on an example zigzag cooling surface as shown in Figure 5-9. In this example, all $\vec{\nabla} l_{p}$ is aligned in $\vec{x}$ direction for illustration purpose. In a general case, small thickness variation is allowed in $\vec{y}$ direction as well in the zigzag optimization method proposed in this work. Thus, the control lines are separated by a resolution distance $\Delta w$ along $\vec{y}$, where the value of $\Delta w$ is negatively proportional to the magnitude of $\vec{\nabla} l_{p}$ in $\vec{y}$ direction. The edge distance $d_{e}$ is set as $12 \mathrm{~mm}$ to obtain a high cooling efficiency on the edge of the zigzag cooling surface without overcooling.

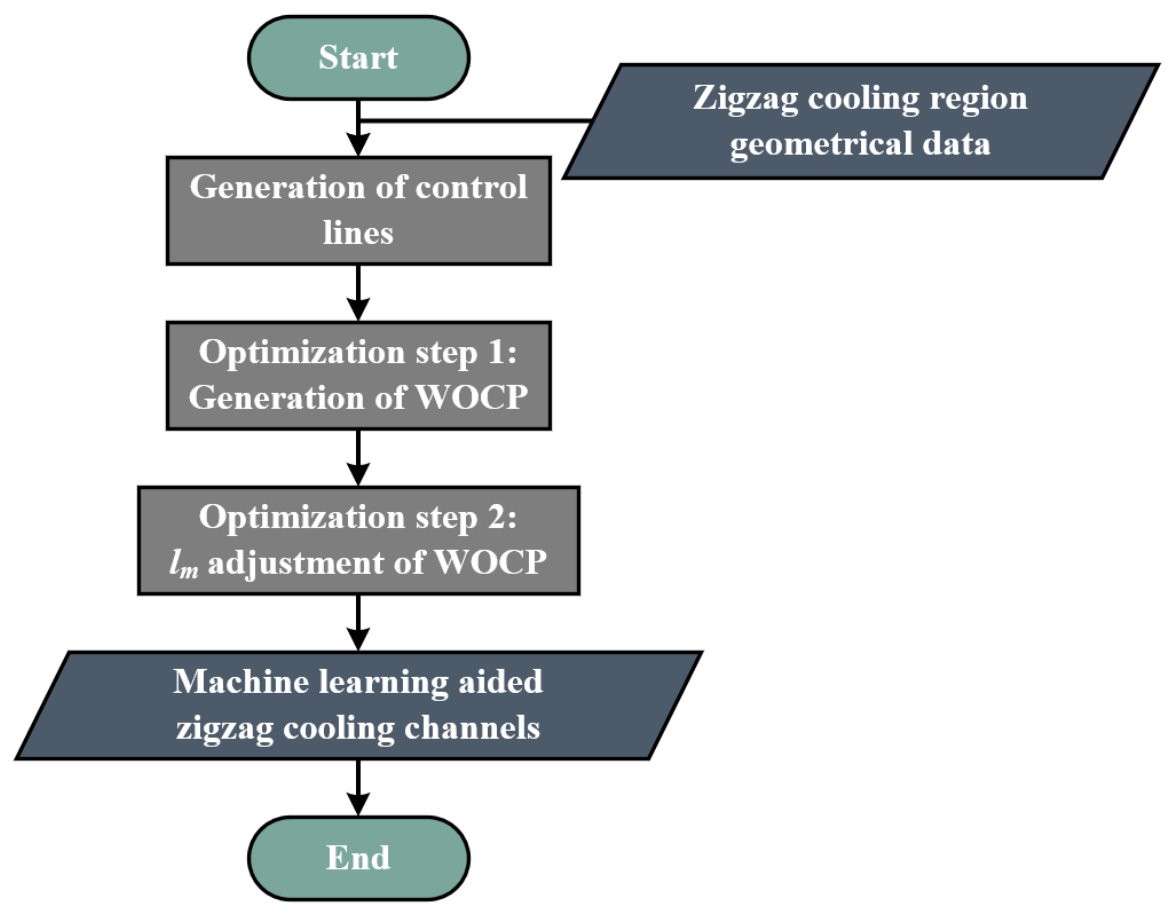

Figure 5-8. The optimization procedure of zigzag cooling channels 


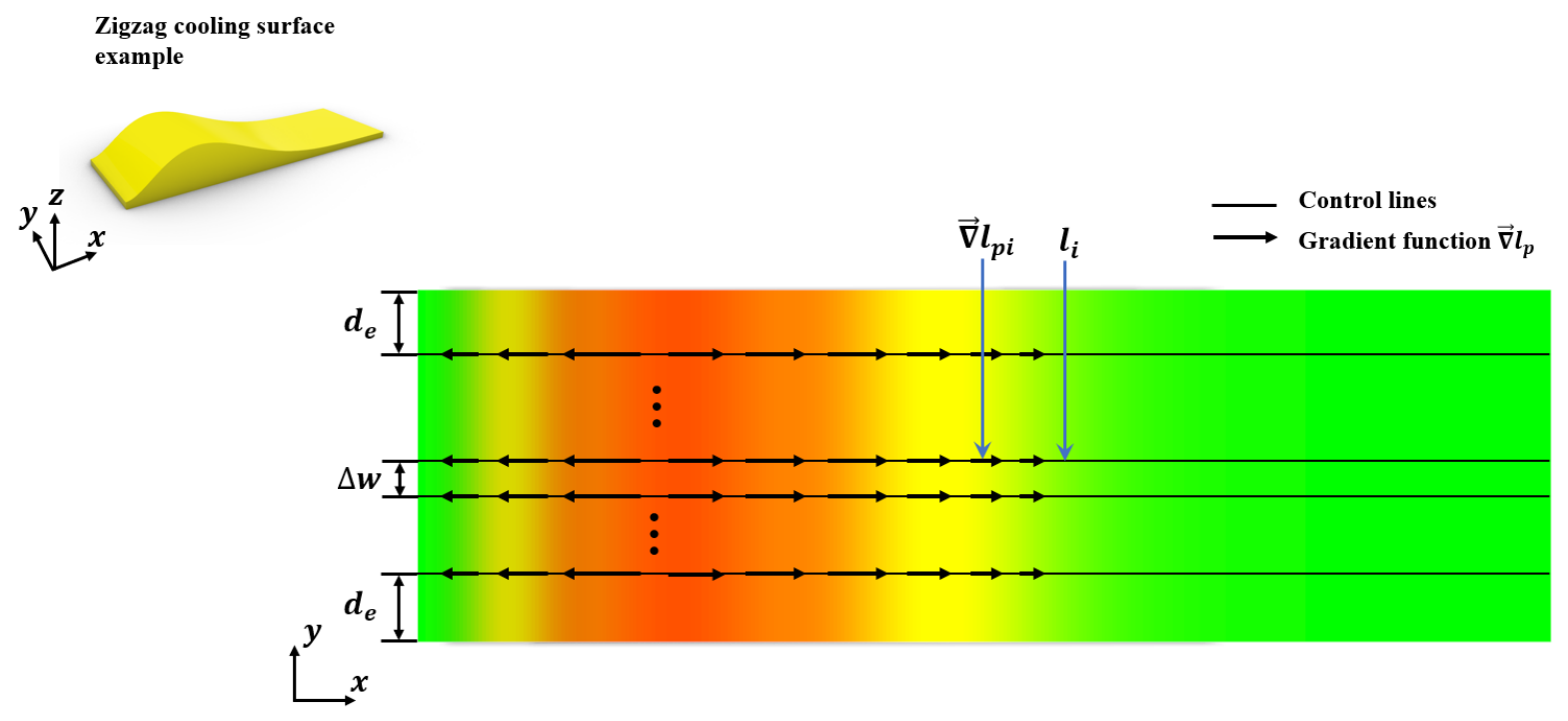

Figure 5-9. An example of a zigzag cooling surface with gradient function and control lines

To generate the zigzag WOCP as shown in Figure 5-10, the control lines are first lofted by a minimum $l_{m_{\min }}=6 \mathrm{~mm}$. On the lofted control lines, any 2 n-point system $\left(p_{1 \sim n}^{(j)} p_{1 \sim n}^{(k)}\right)$ is defined in equation (5-6):

$$
\left\{\left(p_{1 \sim n,}^{(j)} p_{1 \sim n}^{(k)}\right) \mid p_{i}^{(1 \sim m)} \in L(i)\right\}
$$

where $L(i)$ represents the set of all the points on the $i^{t h}$ control line, $n$ is the total number of control lines, and $m$ is the number of points in $L(i)$. Based on the temperature surrogate model for zigzag cooling channels $S M L T M_{z i g-z a g}$, the average temperature $T^{(j, k)}$ for each 2 n-point system is defined in equation (5-7):

$$
T^{(j, k)}=\frac{\sum_{i=1}^{n} T_{i}^{(j, k)}}{n}
$$

where $T_{i}^{(j, k)}$ defined in equation (5-8) is the temperature between $p_{i}^{(j)}$ and $p_{i}^{(k)}$ as shown in Figure 5-10. 


$$
T_{i}^{(j, k)}=\operatorname{SMLTM}_{z i g-z a g}\left(\operatorname{AVG}\left(l_{p i}^{(j)}, l_{p i}^{(k)}\right), W_{i}^{(j, k)}, \operatorname{AVG}\left(l_{m i}^{(j)}, l_{m i}^{(k)}\right)\right)
$$

Based on these calculations, the zigzag optimization step 1 as shown in Table 5-1 is performed to generate zigzag WOCP. During the optimization process, the quantity $m$ and the coordinates of $p_{1 \sim n}^{(j)}$ is updated to minimize $\left|T_{g}-T^{(j, j+1)}\right|$.

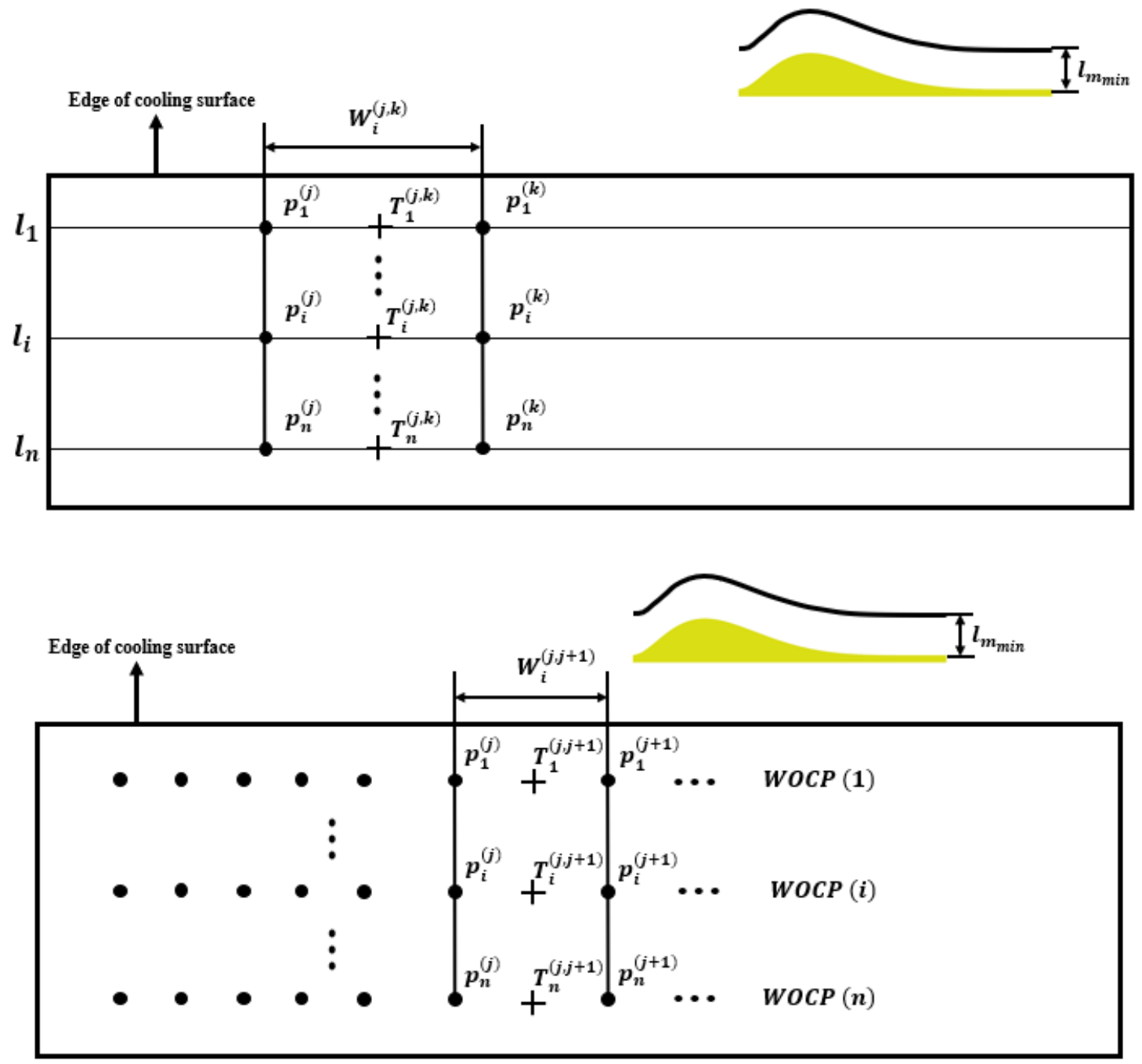

Figure 5-10. Generation of WOCP on a zigzag cooling surface 


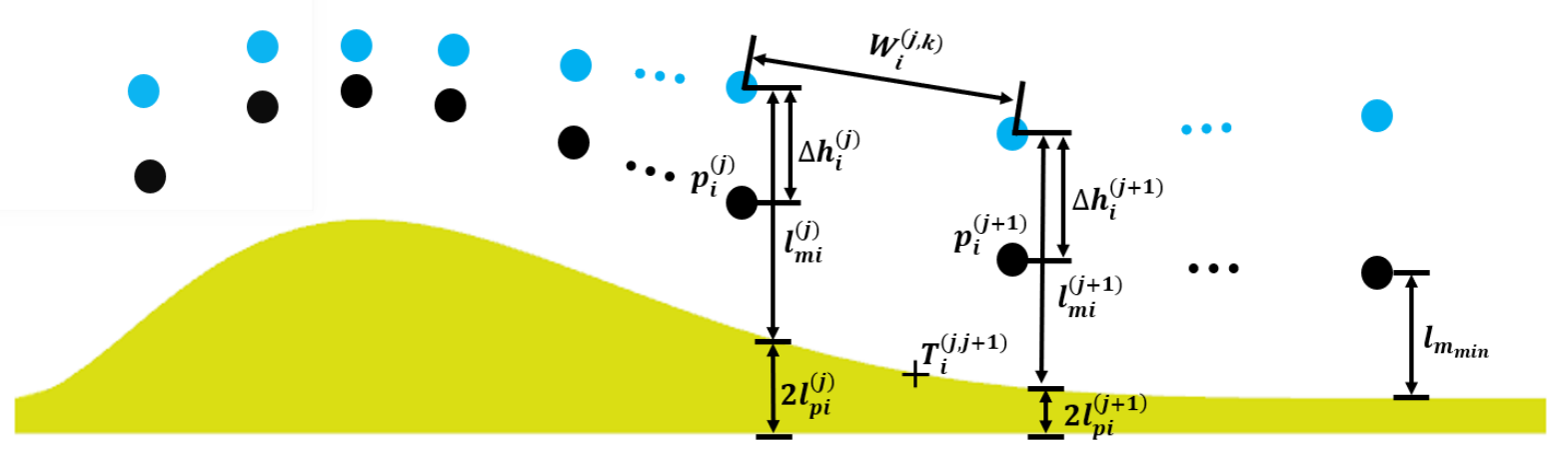

Figure 5-11. Generation of $l_{m}$ - optimized control points of zigzag cooling channels

Finally, the $l_{m}$ - optimized $W O C P$ for a zag-zag conformal cooling channels is generated shown in Figure 5-11. For every control point $p_{i}^{(j)}$ in WOCP, the value of $l_{m i}^{(j)}$ is added by an additional height $\Delta h_{i}^{(j)}$ similar to the optimization process of spiral cooling channels. During the second step of zigzag optimization, the $\Delta h_{i j}$ is optimized to a value so that on each control line $l_{i}$, the temperature difference between $T_{i}^{(j, j+1)}$ and $T_{g}$ is minimized. At the end of the zigzag cooling channels optimization, the $l_{m}$ - optimized WOCP are connected in a proper zigzag sequence to obtain the final machine learning aided zigzag cooling channels as shown in Figure 5-12.

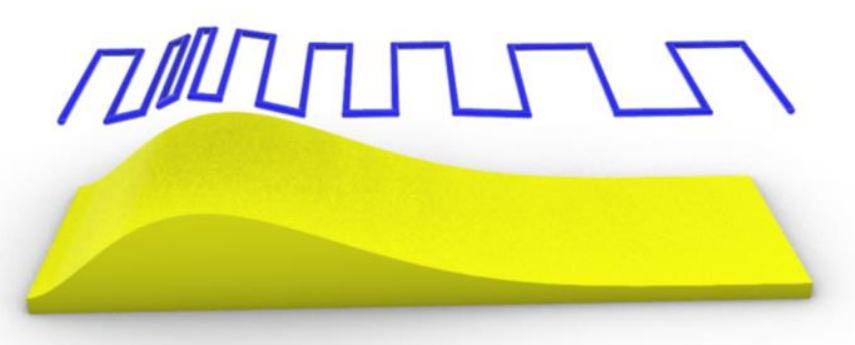

Figure 5-12. Machine learning aided zigzag cooling channel 


\subsection{Optimization of porous cooling channels}

An example of optimized porous channels is shown in Figure 5-13. To design the machine learning aided porous channels, the porosity of the cooling channels $\phi_{i}$ should be optimized with respect to the the half-part thickness $l_{p i}$ for each region $R_{i}$. The objective function during the porous optimization process is the temperature difference $\Delta T_{i}$ between the machine learning predicted temperature $S M L T M_{\text {porous }}\left(l_{p i}, \phi_{i}\right)$ and $T_{g}$ as provided in equation (5-9):

$$
\Delta T_{i}=\left|T_{g}-S M L T M_{\text {porous }}\left(l_{p i}, \phi_{i}\right)\right|
$$

During the optimization iteration, the objective function $\Delta T_{i}$ is minimized by updating $\phi_{i}$ as shown in Table 5-1. Based on the optimized $\phi_{i}$, the machine learning aided porous channels can be generated for each porous cooling region as shown in Figure 5-13.

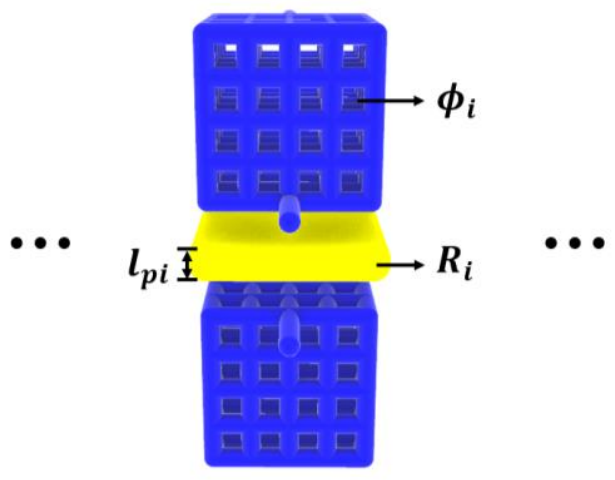

Figure 5-13. Machine learning aided porous cooling channel

\subsection{Optimization of CPS cooling channels}

In this section, the proposed CPS optimization strategy is explained with a swimming pedal case study, where the mathematical representation of the optimization (i.e. objective function, etc.) is provided in Table 5-1, and the optimization procedure of the design parameters is provided in Figure 5-14. 


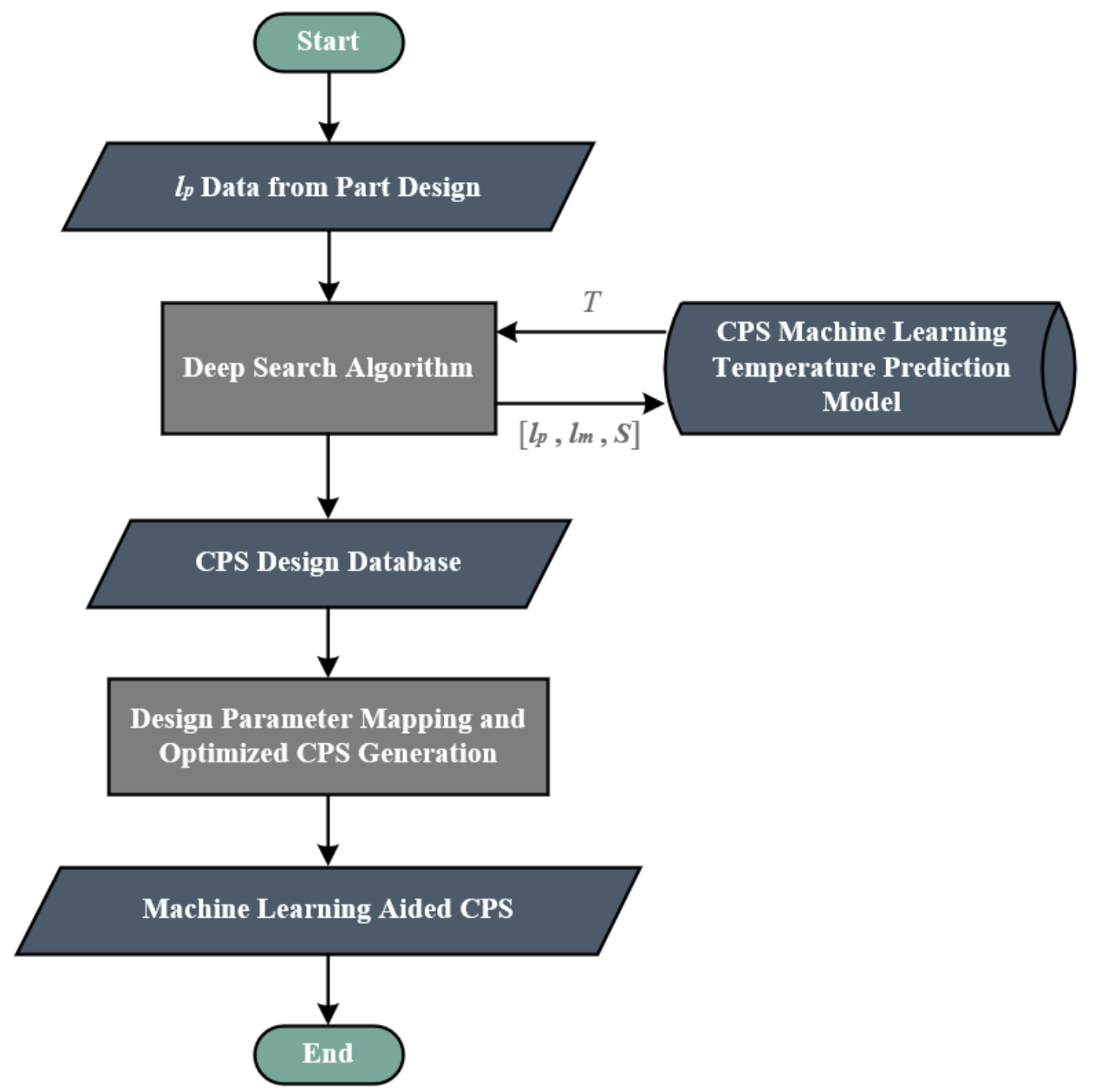

Figure 5-14. The optimization procedure of CPS

First, the range of $l_{p}$ for the given part design evaluated. For the swimming pedal case study, the $l_{p}$ is varying from $0.7 \mathrm{~mm}$ to $5 \mathrm{~mm}$. Then, these $l_{p}$ values are imported into a deep search algorithm to find the optimized corresponding channel design parameters. The flowchart of this algorithm is provided in Figure 5-15. For each $l_{p}$ value from the case study, the ranges of $S$ and $l_{m}$ are first divided into small intervals by a given number of steps $n$, where the initial limits of $S$ and $l_{m}$ are set as $[12 \mathrm{~mm}, 30 \mathrm{~mm}]$ and $[6 \mathrm{~mm}, 30 \mathrm{~mm}]$. Then, the program calculates the corresponding part temperature $T_{(i, j)}$ for each combination of the $S$ and $l_{m}$ intervals from the machine learning model as shown in equation (5-10):

$$
T_{(i, j)}=M\left(S_{i}, l_{m j}, l_{p}\right)
$$




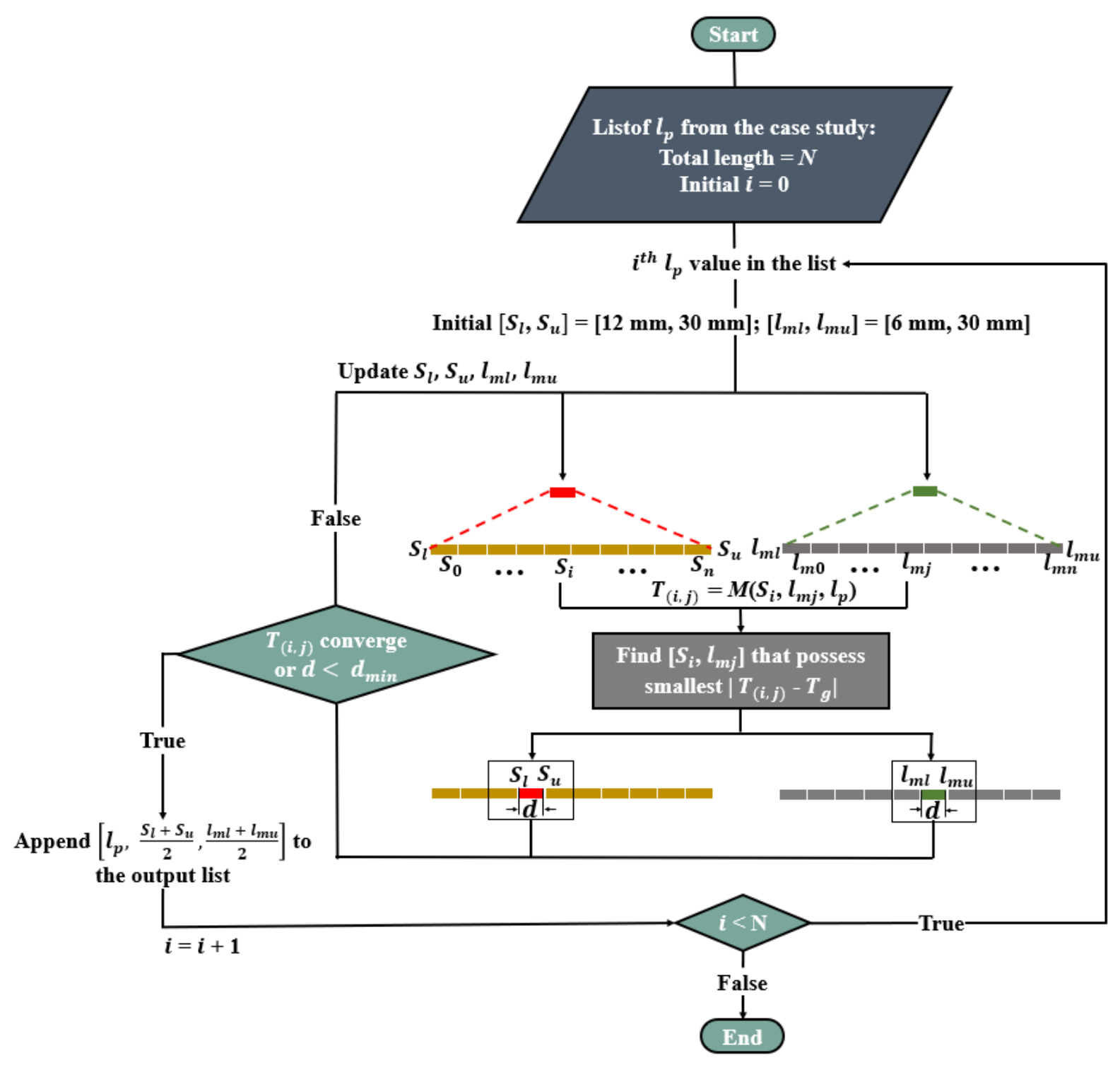

Figure 5-15. A deep search strategy during the optimization

where $S_{i}$ is the $\mathrm{S}$ value at the middle of the $i^{\text {th }} \mathrm{S}$ interval, $l_{m j}$ is the $l_{m}$ value at the middle of $j^{\text {th }} l_{m}$ interval, $M$ represents the surrogate temperature prediction model, and $T_{(i, j)}$ is the predicted resulting part surface temperature for the combination of $i^{\text {th }} \mathrm{S}$ interval and $j^{\text {th }} l_{m}$ interval. An optimal $\left[S, l_{m}\right]$ combination is found among these intervals, which provides the $T_{(i, j)}$ which is the closest to the target temperature $T_{g}$. Afterwards, the program updates the upper and lower limits of $S$ and $l_{m}$ to the limits of the found $S$ and $l_{m}$ intervals as shown in equations (5-11) and (5-12): 


$$
\begin{gathered}
S_{i l} \rightarrow S_{l}, S_{i u} \rightarrow S_{u} \\
l_{m j l} \rightarrow l_{m l}, l_{m j u} \rightarrow l_{m u}
\end{gathered}
$$

where $S_{l}, S_{u}$ and $l_{m l}, l_{m u}$ are the lower and upper limits of $S$ and $l_{m}$ at the beginning of each iteration, $S_{i l}, S_{i u}$ and $l_{m j l}, l_{m j u}$ are the lower and upper limits of the $i^{\text {th }} S$ interval and $j^{\text {th }} l_{m}$ interval which provides $T_{(i, j)}$ that is closest to $T_{g}$, respectively. Consequently, the range of the $S$ and $l_{m}$ is updated to a smaller value $d$ shown in Figure 5-15. The program will then iterate and decompose these new ranges by $n$ again and search for a more precise $\left[S, l_{m}\right]$ combination. The iteration stops once the predicted temperature converges to the value that is closest to $T_{g}$, or the new ranges have already reached highest resolution $\left(d<d_{\min }\right)$. The time efficiency of this search strategy is summarized and compared with nested loop searching approach method in Table 5-2, where $n$ is the number of steps to divide the given interval, $l$ is the initial range of the design parameters, $d_{\min }$ represents the minimum interval allowed during the optimization, $N$ is the number of different $l_{p}$ values. Theoretically, the deep search algorithm is more time efficient as $m$ scales up. Based on this new search algorithm, a database is generated, which can efficiently provide the optimized [ $S$, $\left.l_{m}\right]$ for every thickness values of the given part design. These optimized design parameters are then mapped onto the cooling surface as shown in Figure 5-16, and the final machine learning aided CPS design is constructed as shown in Figure 5-17. As a result, the machine learning aided design of CPS has a larger $\mathrm{S}$ and $l_{m}$ values at the thinner regions of the part (i.e. regions with $x \in$ [0 mm, $100 \mathrm{~mm}]$ in Figure 5-16b), which prevents the temperature variance caused by the overcooling on those regions.

Table 5-2. Time efficiency of deep search algorithm and nested loop approach

\begin{tabular}{|c|c|}
\hline Algorithm & Worst-case running time \\
\hline Deep search algorithm & $\mathrm{O}\left(N \frac{n^{2}}{m}\right)$, where $m=\frac{l}{d_{\min }}$ \\
\hline Nested loops approach & $\mathrm{O}\left(\mathrm{Nm}^{2}\right)$ \\
\hline
\end{tabular}


(a) Reference geometrical line on the swimming pedal
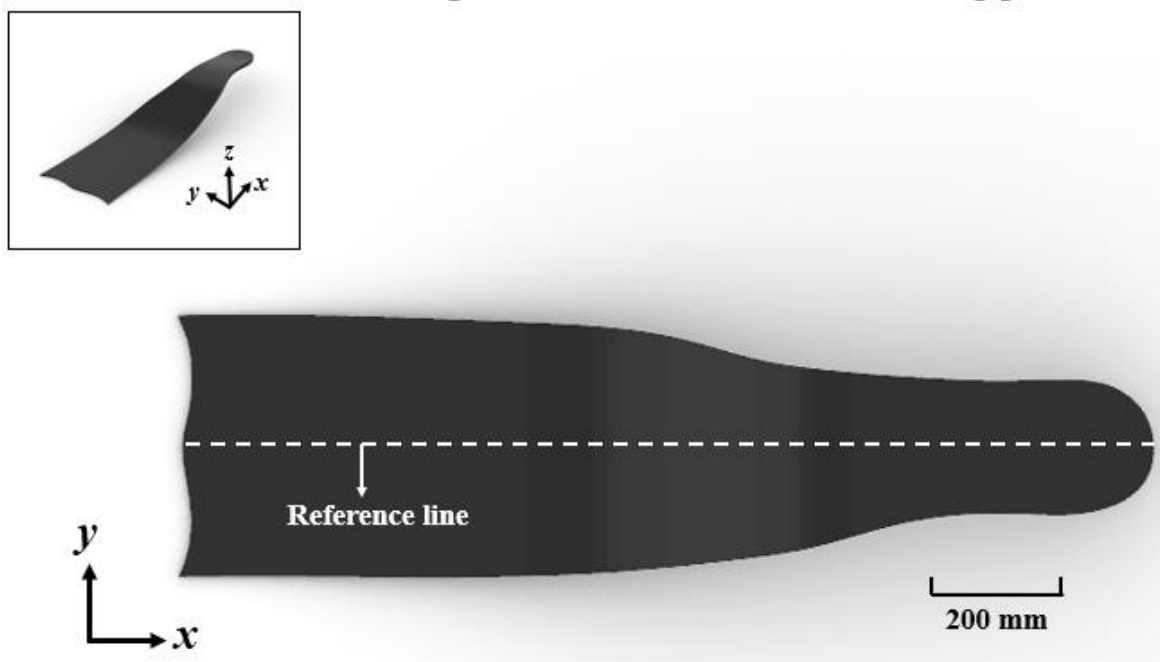

(b) Design data on the reference geometrical line
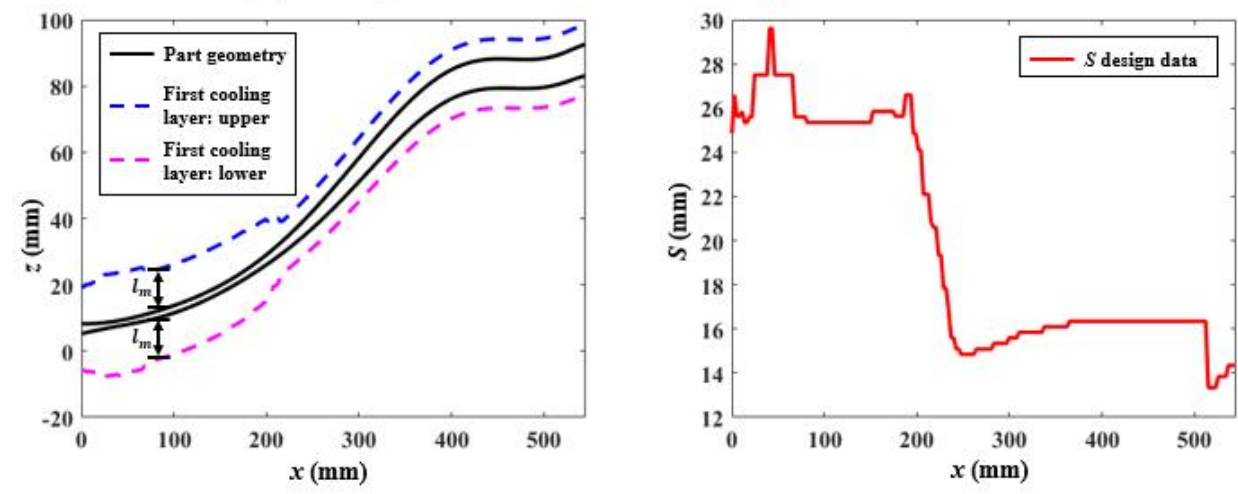

Figure 5-16. Design data and the optimized design data

(a) Perspective view

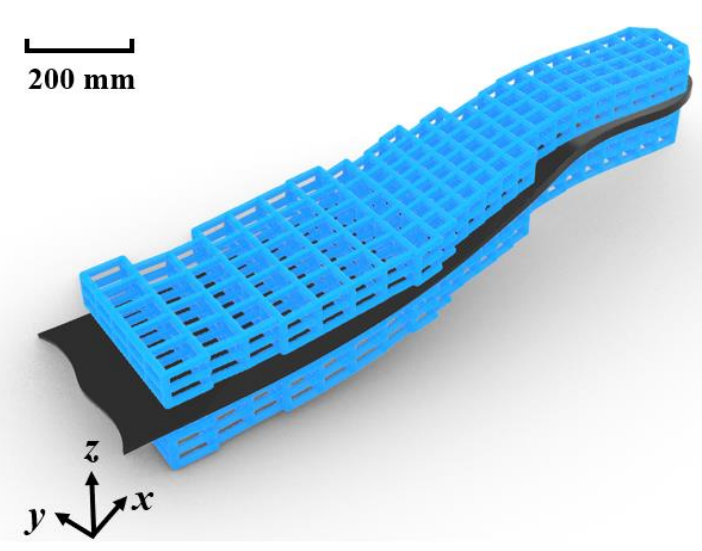

(b) Top view

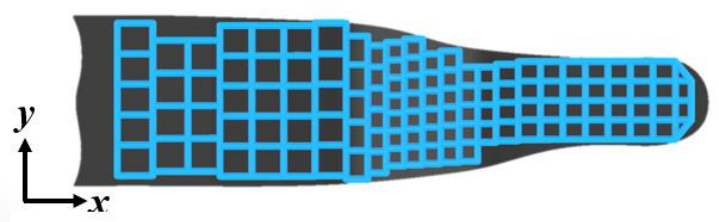

(c) Side view

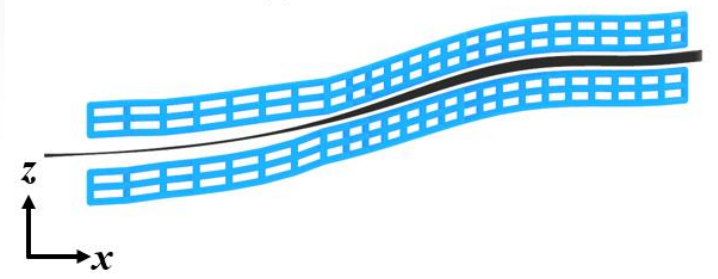

Figure 5-17. Machine learning aided CPS 


\subsection{Summary}

In this chapter, different TVM methods are proposed and discussed for zigzag, spiral, porous, and CPS cooling topologies to generate the final MLACCD channels. Variety types of optimization strategies are applied for these topologies due to their distinct geometrical designs. For the zigzag and spiral cooling channels, the pitch width $W$ is first optimized to generate WOCP. Then, the distances from each point of WOCP to the mold surface are then adjusted to obtain an optimal $l_{m}$ value for those control points. Due to this sequential optimization strategy, the MLACCD provides a larger width and shorter length of the zigzag and spiral cooling channels, which not only results in a decreased temperature variance, but also effectively reduces the coolant pressure drop. For the porous and CPS cooling channels where the pressure drop is already very low, the most optimized combinations of the design parameters are found to reduce the temperature variance without any optimization priorities. For each thickness value of the part, the porosity $\phi$ is optimized for porous cooling channels, and a deep search strategy is applied for CPS to efficiently found optimized $\left[S, l_{m}\right]$ sets. Based on the TVM tool developed in this work, the final MLACCD channels can be generated. In the next chapter, different case studies are discussed to validate the effectiveness of MLACCD. 


\section{CHAPTER 6 \\ Validation case studies and implementation of MLACCD software}

In this chapter, case studies are provided in Section 6.1 to validate the effectiveness of the proposed MLACCD method. In addition, the implementation of the MLACCD software is provided in Section 6.2, where the main functions, graphical user interface (GUI), and the expected program inputs and outputs are discussed in detail.

\subsection{Validation case studies}

In this section different design cases including a freeform surface, a shoe sole, and a swimming pedal as shown in Figure 6-1 are studied to validate the proposed method. The cooling simulations are using conventional conformal cooling channels (CCCD) and machine learning aided conformal cooling channels (MLACCD) on Moldflow Advisor ${ }^{\circledR}$ simulation software. The input parameters for those case studies are provided in Table 6-1, where they are kept the same for all case studies with CCCD and MLACCD cooling systems for comparison purposes.

(a) Freeform part surface

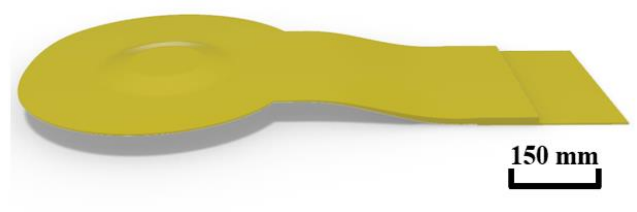

(b) Shoe sole

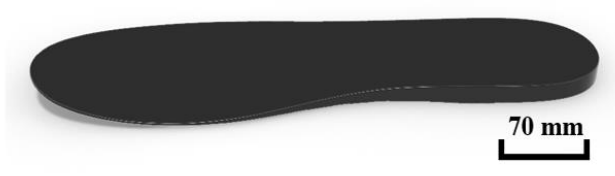

(c) Swimming pedal

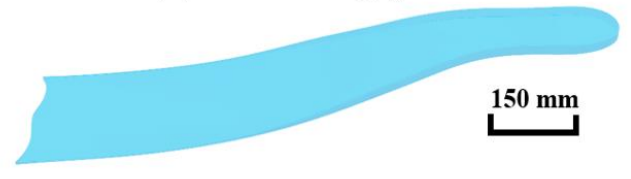

Figure 6-1. The part designs of case studies 
Table 6-1. Parameters of injection molding cooling simulation for the case studies

\begin{tabular}{|c|c|}
\hline Mold material & H13 Tool Steel \\
\hline Initial mold temperature & $323.15 \mathrm{~K}$ \\
\hline Part material & Generic PP \\
\hline Melt temperature & $493.15 \mathrm{~K}$ \\
\hline Coolant type & Water \\
\hline Coolant inlet temperature & $298.15 \mathrm{~K}$ \\
\hline Average coolant flow rate & $5 \mathrm{lit} / \mathrm{min}$ \\
\hline
\end{tabular}

To compare the resulting temeperature variance and the enery efficiency of the MLACCD and CCCD cooling systems, the temperature distribution and pressure drop simulation results are first analyzed on a freeform surface and a shoe sole case study with zigzag, spiral, and porous cooling channels. The results indicate that a significant reduction of temperature variance, and a smaller pressure drop is achieved by the MLACCD compared to CCCD. Based on the validated design method, an innovative machine learning aided CPS is developed for a swimming pedal case study to further validate the generality and effectiveness of the proposed method on a more novel conformal cooling system design. As a result, the machine learning aided CPS also exhibits great cooling performances in terms of minimizing temeprature variance compared to convenitonal CPS.

\subsubsection{Freeform part surface and shoe sole}

To validate the effectiveness of MLACCD channels, two design cases containing conventional conformal cooling channels and MLACCD channels are provided. In the first case study, a plastic part with a freeform surface is used. Figure 6-2a shows the cooling channels generated by the conventional conformal cooling design method, while Figure 6-2b represents the cooling channels designed based on the MLACCD method proposed in this paper. The cooling channels generated by different methods are imported to Autodesk Moldflow Advisor ${ }^{\circledR}$, and the parameters used in the simulation are summarized in Table 6-1. The results of the simulations for the first design case are summarized in Table 6-2, and it will be carefully discussed in the following paragraphs. 
(a) Conventional conformal cooling channels

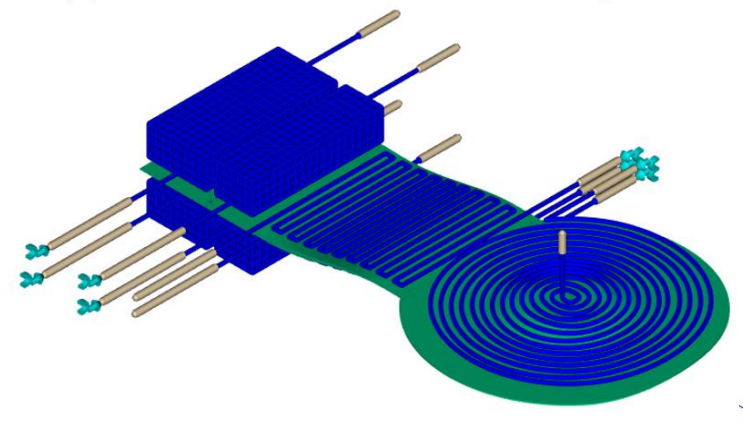

$500 \mathrm{~mm}$ (b) MLACCD channels

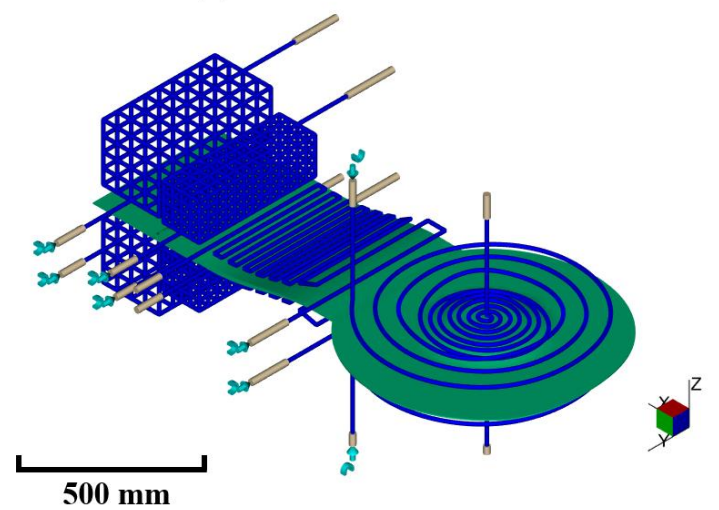

Figure 6-2. Comparison of CCCD and MLACCD channels

Table 6-2. A summary of simulation results for the first design case including: temperature variance at critical locations, $\sigma_{T}$, and the pressure drop

\begin{tabular}{|l|l|l|l|l|l|l|}
\hline \multirow{2}{*}{$\begin{array}{l}\text { Design } \\
\text { Area }\end{array}$} & \multicolumn{2}{|l|}{$\begin{array}{l}\text { Temperature variance at } \\
\text { critical locations }\left({ }^{\circ} \mathrm{C}\right)\end{array}$} & \multicolumn{2}{|l|}{$\sigma_{T}\left({ }^{\circ} \mathrm{C}\right)$} & \multicolumn{2}{l|}{ Pressure drop (kPa) } \\
\cline { 2 - 7 } & MLACCD & $\begin{array}{l}\text { Conventional } \\
\text { conformal } \\
\text { cooling } \\
\text { design }\end{array}$ & MLACCD & $\begin{array}{l}\text { Conventional } \\
\text { conformal } \\
\text { cooling } \\
\text { design }\end{array}$ & MLACCD & $\begin{array}{l}\text { Conventional } \\
\text { conformal } \\
\text { cooling } \\
\text { design }\end{array}$ \\
\hline Spiral & 0.54 & 8.12 & 0.36 & 2.85 & 125 & 244.81 \\
\hline Zigzag & 2 & 7.43 & 0.73 & 2.57 & 226.62 & 269.67 \\
\hline Porous & 4.2 & 5.04 & 1.93 & 2.22 & 909 & 1388.1 \\
\hline Total & \multicolumn{2}{|l|}{ Not applicable } & 1.18 & 2.83 & \multicolumn{2}{l|}{ Not applicable } \\
\hline
\end{tabular}

The part surface temperature and the temperature variation simulation results are shown in Figure 6-3. Comparing to conventional conformal cooling channels, a significant reduction of temperature variance along the overall cooling surface within the same injection cycle time is achieved by MLACCD as shown in Figure 6-3c and Figure 6-3d. For MLACCD channels, the temperature differences among the regions with different thickness values as shown in Figure 6-3a are $0.54^{\circ} \mathrm{C}, 2^{\circ} \mathrm{C}$, and $4.2^{\circ} \mathrm{C}$ for spiral, zigzag, and porous cooling surface, respectively. While for conventional cooling channels, the corresponding temperature variances are $8.12^{\circ} \mathrm{C}, 7.43^{\circ} \mathrm{C}$, and $5.04{ }^{\circ} \mathrm{C}$ as shown in Figure 6-3b. Thus, the temperature variations are reduced by $93 \%, 73 \%$, and 
$17 \%$ for spiral, zigzag, and porous design areas, respectively. There are two reasons that the cooling result achieved by MLACCD has a smaller temperature variation. First, the conventional conformal cooling channels are not conformal to part thickness comparing to MLACCD channels.

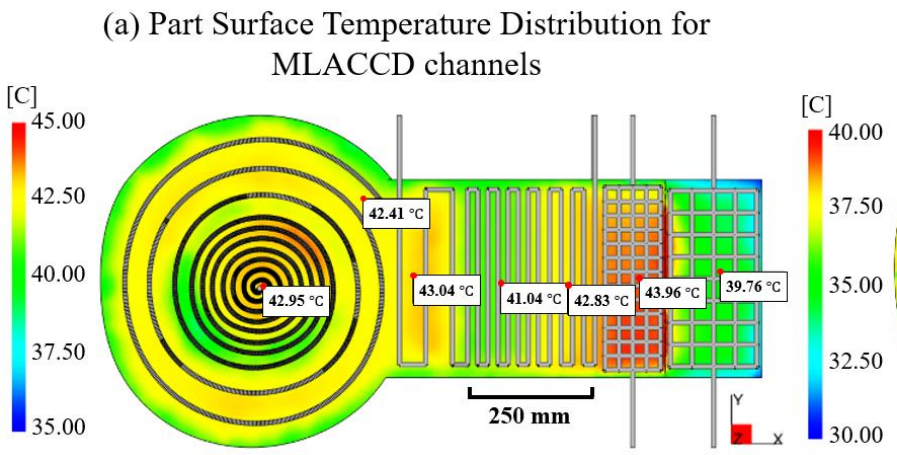

(c) Part Surface Temperature Variance for

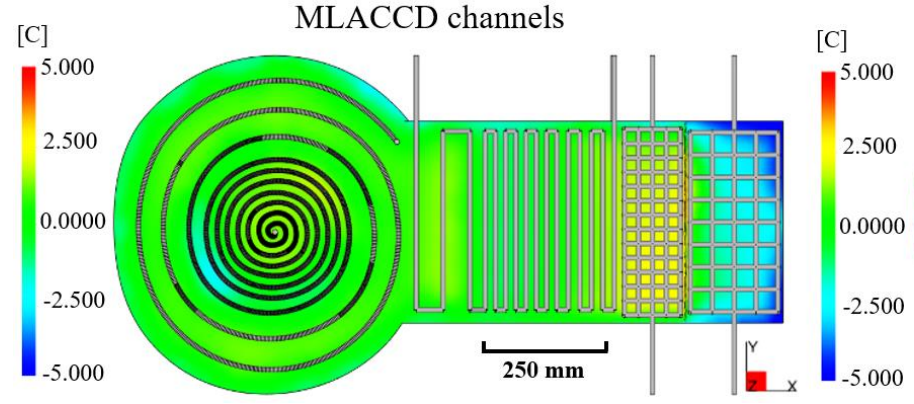

(b) Part Surface Temperature Distribution for conventional conformal cooling channels

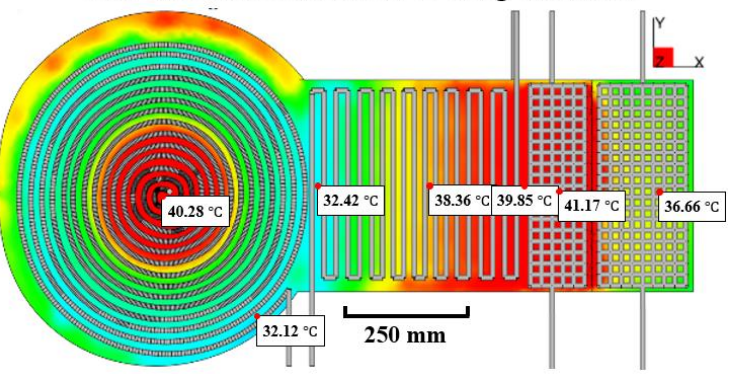

(d) Part Surface Temperature Variance for conventional conformal cooling channels

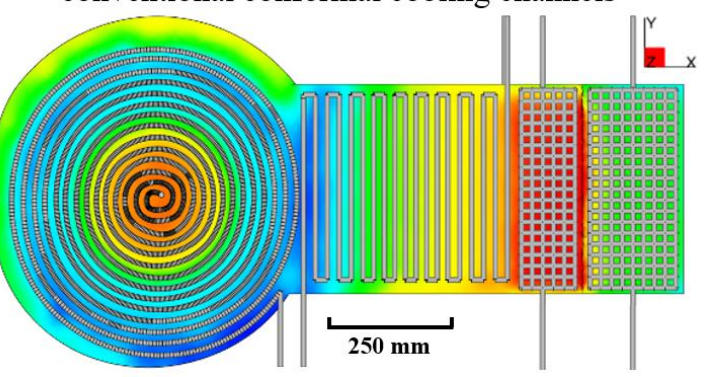

Figure 6-3. The temperature distribution and the temperature variance of the part surface achieved by MLACCD channels and conventional conformal cooling channels

For the cooling regions with different thickness values, unequal amount of heat needs to be carried away through the cooling passageway to reach a uniform part surface temperature after the cooling process. For conventional conformal cooling design, the cooling efficiency is uniform along the part surface. This results in a higher temperature variance on the thicker regions of the part comparing to MLACCD, since the MLACCD channels are conformal to not only the part surface but also the thickness distribution of the part geometry. Second, the coolant temperature differences between the inlets and outlets of the cooling system are reduced in MLACCD. As shown in Figure 6-4, the maximum temperature rise between coolant inlet and outlet is $3.65^{\circ} \mathrm{C}$, 
while the value for conventional designed conformal cooling channels is $4.02^{\circ} \mathrm{C}$. This smaller coolant temperature rise is due to the reduced total length of channels in MLACCD.

(a) Coolant temperature of MLACCD cooling channels

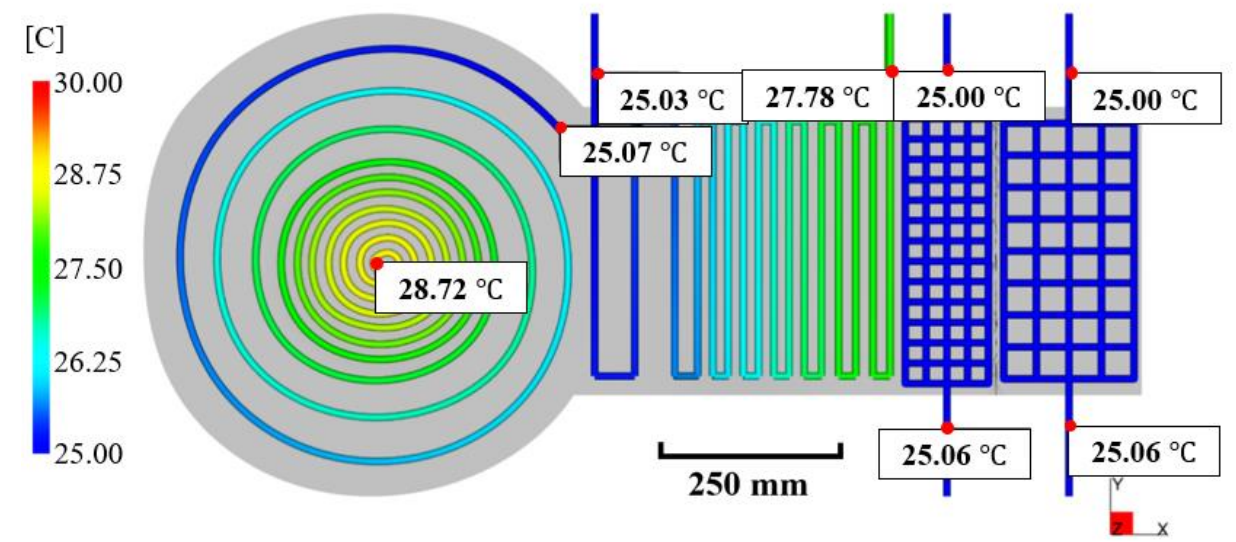

(b) Coolant temperature of conventional conformal cooling channels

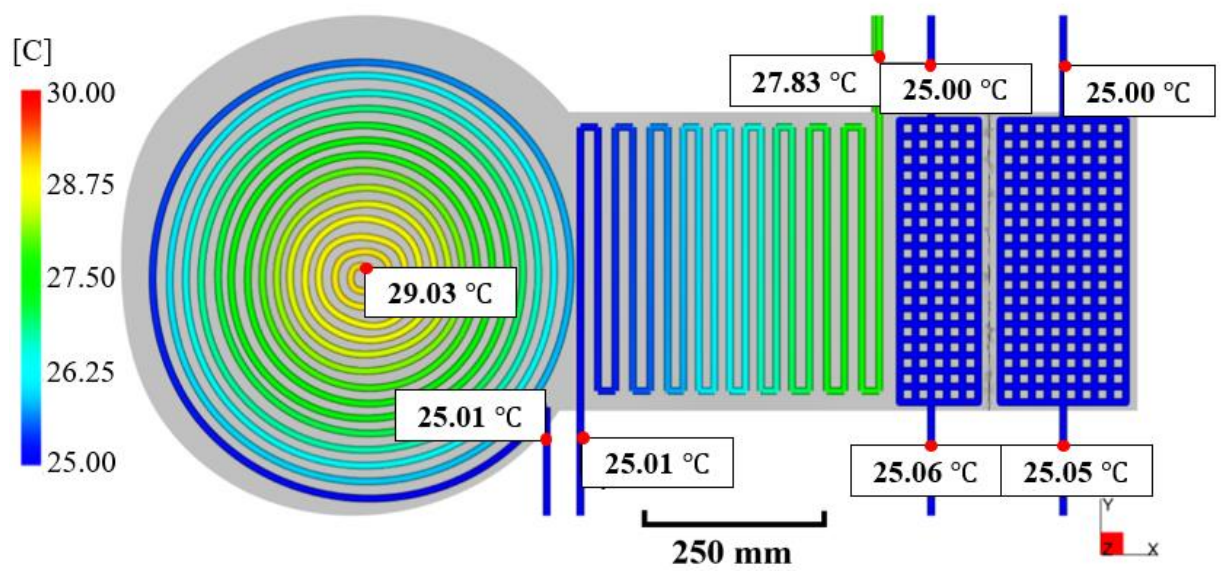

Figure 6-4. The coolant temperature distribution of the cooling channels for MLACCD channels and conventional conformal cooling channels

To further compare the part surface temperature variation of two design cases, the surface temperature together with the part thickness along the center line is provided in Figure 6-5. It is found that the part surface temperature for the conventional conformal cooling system exhibits a higher sensitivity to the thickness variation, while this effect is minimized with MLACCD channels. To quantify this result, the standard deviations of part surface temperature $\sigma_{T}$ for 
different cooling regions along the center line are calculated and summarized in Table 6-2 for each design of cooling channels. For the total cooling surface of MLACCD channels, the $\sigma_{T}$ values are reduced by $58 \%$. Specifically, the $\sigma_{T}$ values for MLACCD channels are $87 \%, 72 \%$, and $13 \%$ compared with conventional conformal cooling channels. However, there are still variations between the machine learning prediction of part surface temperature and the simulation result as shown in Figure 6-5. Most of the variations are within $1^{\circ} \mathrm{C}$ temperature variation as programmed in the optimization algorithm, while some of the part surface temperatures observed at the porous cooling area have exceeded this limit. These variations are caused by the temperature rise of the coolant and the unevenly distributed coolant flow rates. As shown in Figure 6-4, the temperatures of the coolant are higher at the positions which are closer to the coolant outlets. In addition, the flow rate is quite un-uniform for the porous conformal cooling channels as shown in Figure 6-6, which contributes to the variations of simulation and machine learning prediction results of part surface temperature.

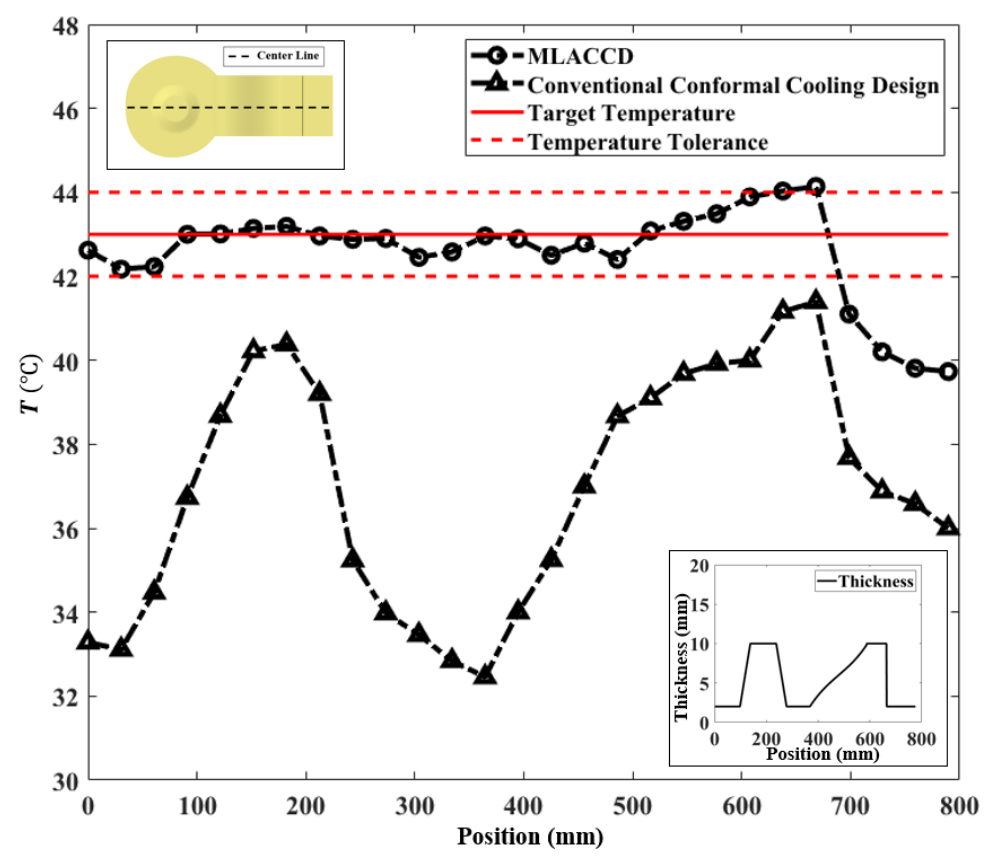

Figure 6-5. The part surface temperature distribution along the center of the part geometry for MLACCD channels and conventional conformal cooling channels 


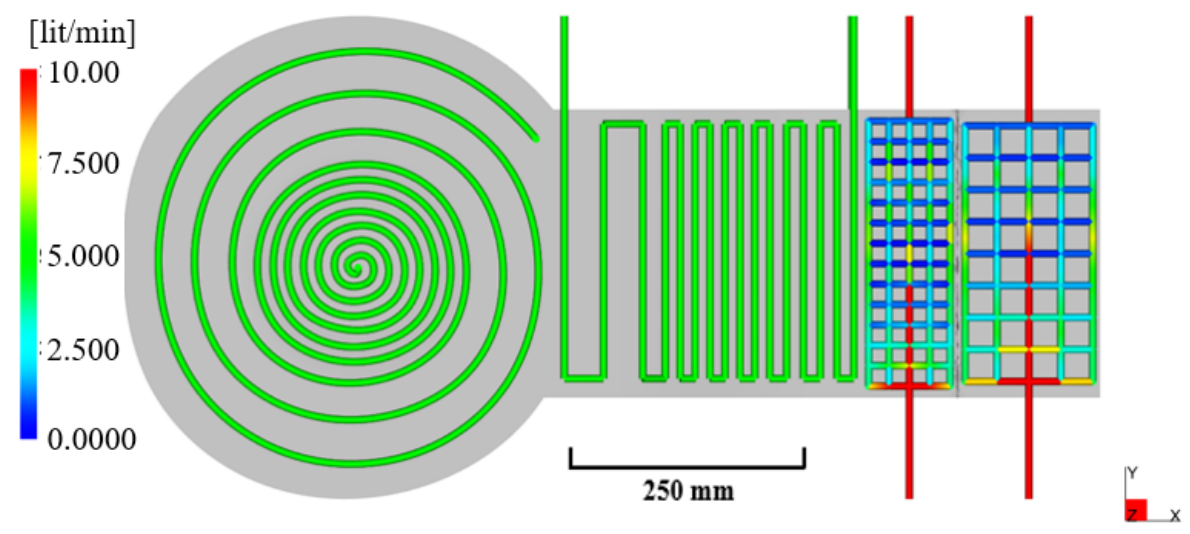

Figure 6-6. The coolant flow rate distribution of MLACCD channels

To compare the energy efficiency of the cooling systems from these two design cases, simulations are performed to obtain the cooling passageway pressure drop. The result of this simulation is again summarized in Table 6-2. The result shows that the mold with MLACCD channels has less energy consumption during the injection molding process since it requires less power to pump the coolant with sufficient inlet pressure. Specifically, the pressure drops of MLACCD spiral, zigzag, and porous channels are 49\%, 16\%, and 35\% lower compared with conventionally designed conformal cooling systems. The cause of the smaller pressure drops of MLACCD channels is their significantly reduced cooling channel lengths. For instance, a larger pitch width is found for the MLACCD spiral cooling channels at the thinner part region, which results in less dense cooling channels, and hence a reduced coolant pressure drops.

(a) Shoe sole

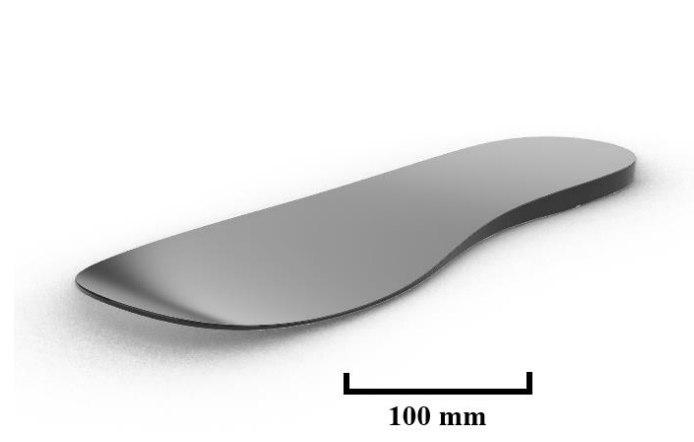

(b) MLACCD channels for the shoe sole

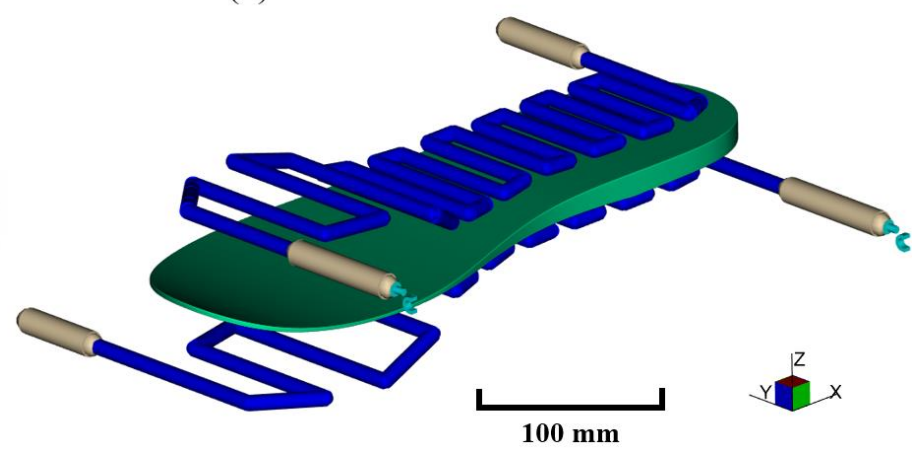

Figure 6-7. Shoe sole and its MLACCD channels 

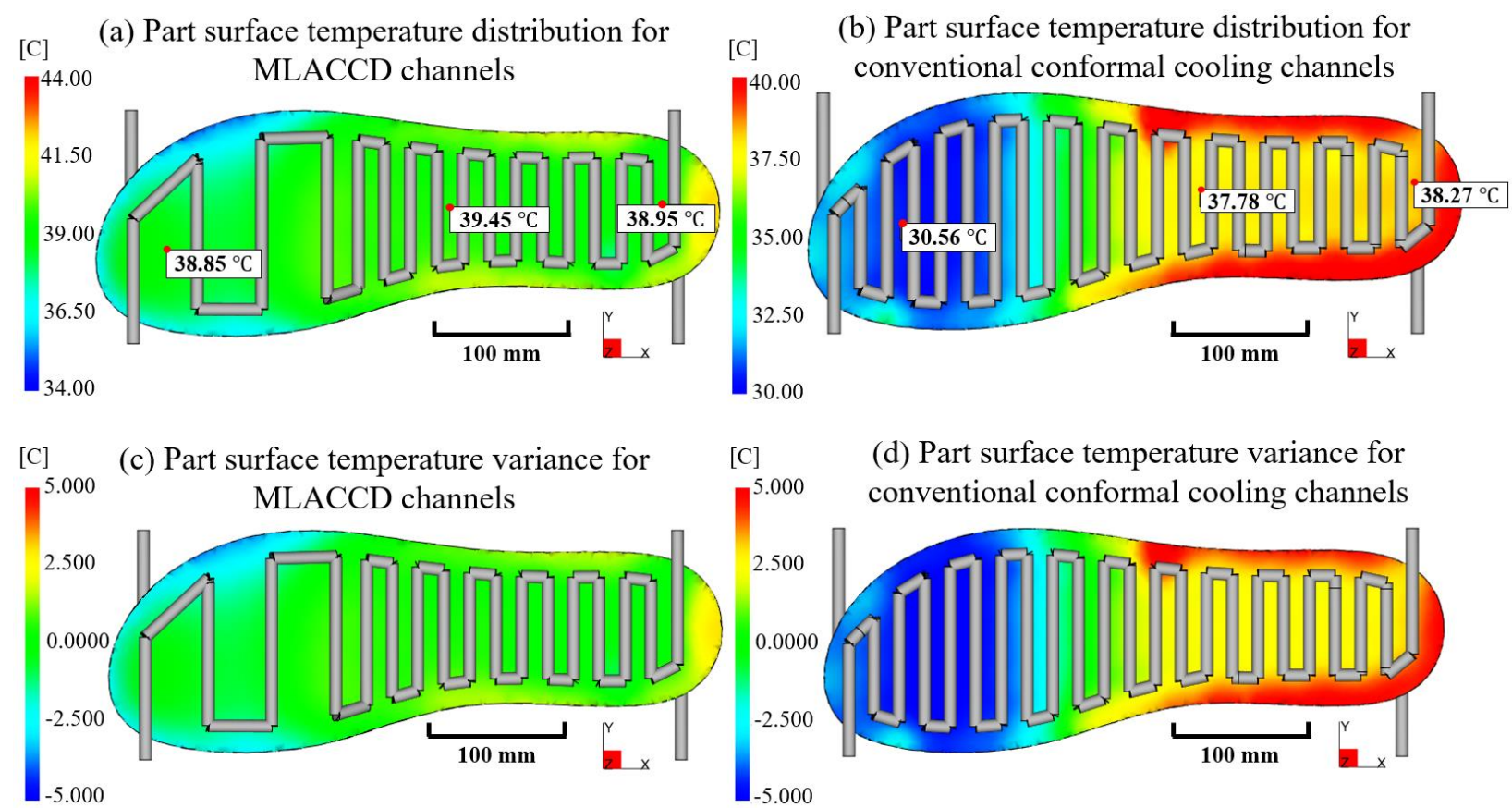

Figure 6-8. The temperature distribution and the temperature variance of the part surface achieved by MLACCD channels and conventional conformal cooling channels

In the second case study, a shoe sole with a curved surface as shown in Figure 6-7 is selected to validate the generality of MLACCD proposed in this study. The cooling performance is analyzed through Autodesk Moldflow Advisor ${ }^{\circledR}$ simulations. The input parameters of the simulations are kept the same as the rest of the case studies and they are listed in Table 6-1. Based on the thickness distribution of the shoe sole, the zigzag cooling channel topology is selected in this design case. The simulation result of the part surface temperature together with the temperature variation is provided in Figure 6-8. Similar to the first design case, the MLACCD channels provide a significantly part surface temperature variance reduction comparing to the conventional conformal cooling channels. Specifically, the temperature variance among the regions with different thicknesses is decreased by $92 \%$ from $7.71^{\circ} \mathrm{C}$ to $0.6^{\circ} \mathrm{C}$. For the part surface temperature along the center line of the shoe sole as shown in Figure 6-9, the effect of the part thickness variations on the resulting temperature is minimized by MLACCD channels. The $\sigma_{T}$ 


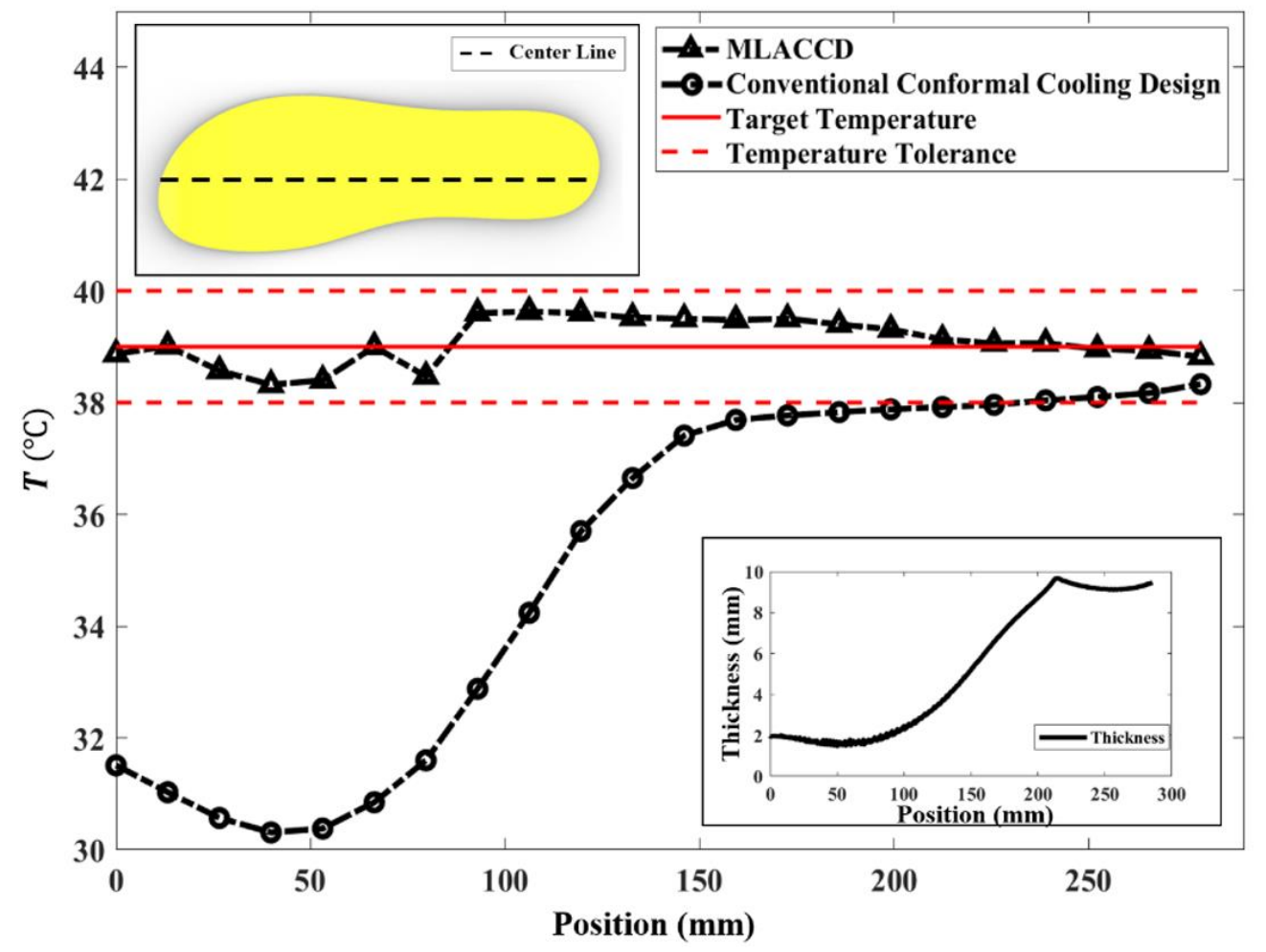

Figure 6-9. The part surface temperature distribution along the center of the part geometry for MLACCD channels and conventional conformal cooling channels

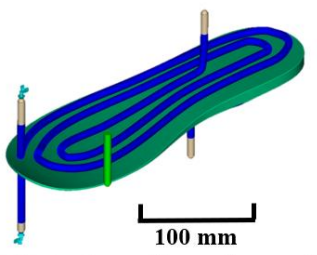

(a) Spiral cooling channels [C]

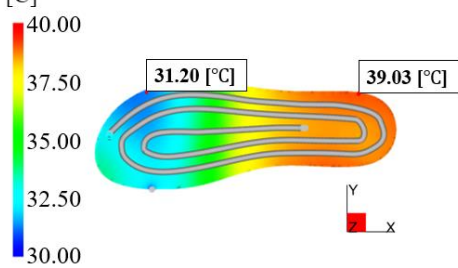

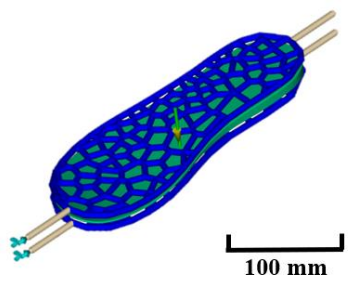

(b) Voronoi cooling channels [C]

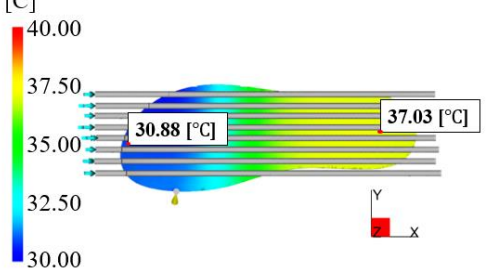

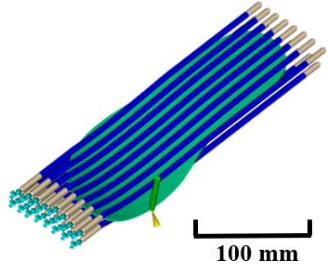

(c) Parallel cooling channels $[\mathrm{C}]$

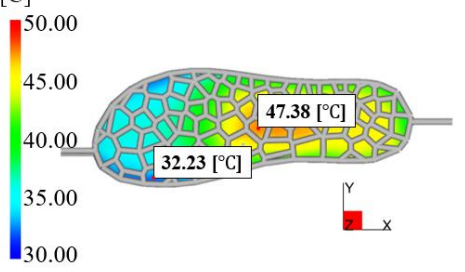

(d) Part surface temperature distribution (e) Part surface temperature distribution (f) Part surface temperature distribution for spiral cooling channels for parallel cooling channels for Voronoi cooling channels

Figure 6-10. State-of-the-art conformal cooling channel topologies

value calculated for MLACCD is $0.39^{\circ} \mathrm{C}$, which is $88 \%$ lower than $3.25^{\circ} \mathrm{C}$ for conventional conformal cooling design. To compare the MLACCD channels with more state-of-the-art 
conformal cooling designs, additional simulations are performed for spiral, parallel, and Voronoi conformal cooling channel topologies given in Figure 6-10a to Figure 6-10c with the same input parameters as shown in Table 6-1. The resulting temperature distributions of those channel topologies are shown in Figure 6-10d to Figure 6-10f. Comparing to $0.6{ }^{\circ} \mathrm{C}$ temperature variance for MLACCD channels, the temperature variances of the part surface for spiral, parallel, and Voronoi conformal cooling channels are $7.83^{\circ} \mathrm{C}, 6.15^{\circ} \mathrm{C}$, and $15.15^{\circ} \mathrm{C}$, respectively. In addition, a reduced coolant pressure drop is achieved by MLACCD. As shown in Figure 6-11, the pressure drop of the coolant is decreased from $246.16 \mathrm{kPa}$ to $178.82 \mathrm{kPa}$ when MLACCD is applied.
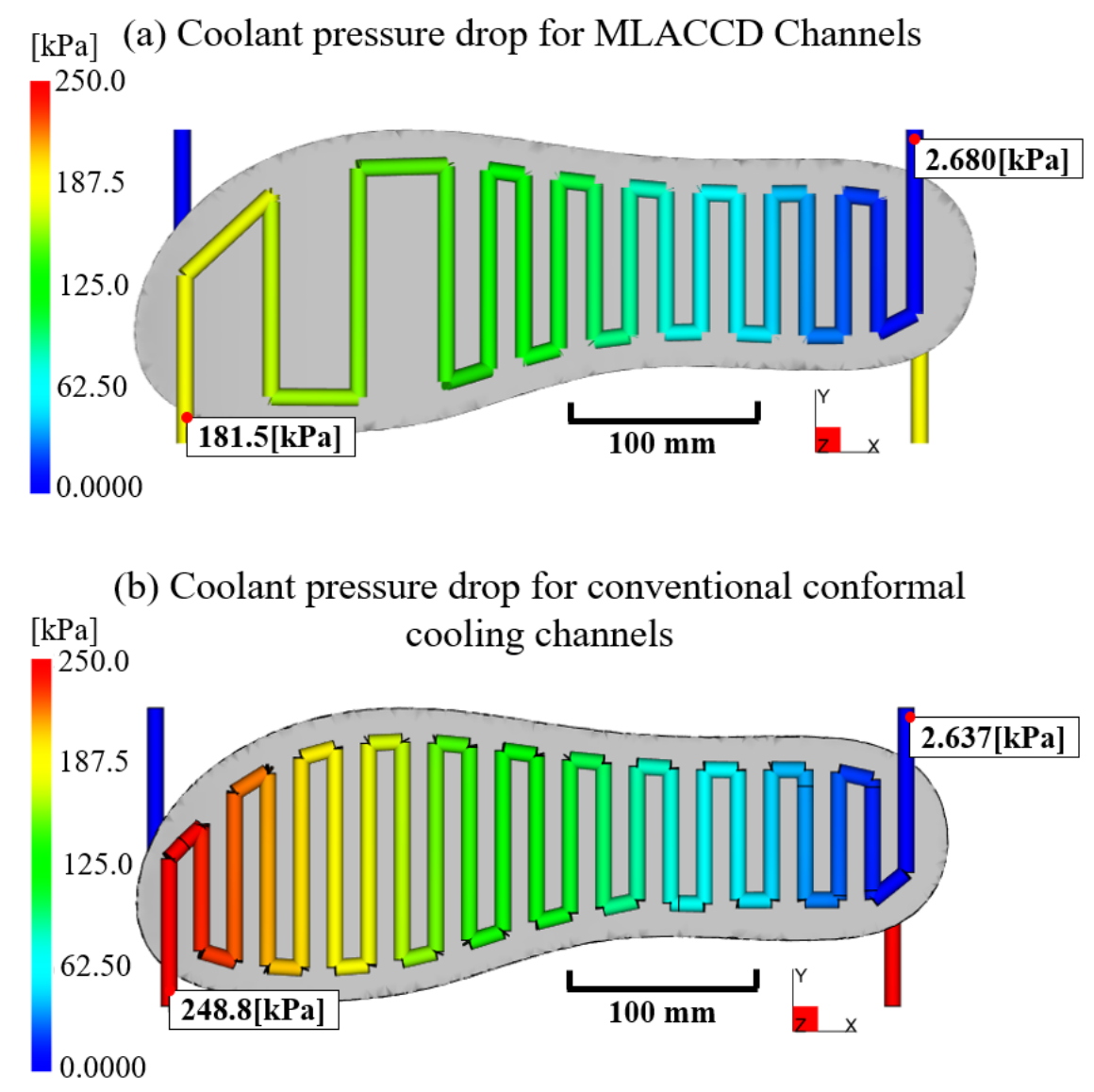

Figure 6-11. Coolant pressure drop for MLACCD channels and conventional conformal cooling channels 


\subsubsection{Swimming pedal}

In this section, cooling simulations are performed on a swimming pedal case study to further validate the effectiveness of the proposed method in novel CPS design using Moldflow Advisor ${ }^{\circledR}$. Figure 6-12 shows the machine learning aided and conventional CPS for a swimming pedal case study. Unlike conventional CPS, the machine learning aided designed CPS exhibits a larger cooling cell size $\mathrm{S}$ and cooling surface to mold surface distance at the part regions with smaller thickness values. This design allows a properly adjusted cooling efficiency to be applied along the part surface. The simulation input parameters are provided in Table 6-1, where these parameters are kept the same for both designs for comparison purpose. The results of the simulations are shown in Figure 6-13. Comparing to conventional CPS, the resulting part temperature distribution for the machine learning aided CPS is more uniform. As shown in Figure 6-13a, the part temperature variance at critical locations for the machine learning aided CPS is $2.07{ }^{\circ} \mathrm{C}$, which is $76 \%$ lower than $8.48^{\circ} \mathrm{C}$ for the conventional CPS.

(a) Machine learning aided CPS

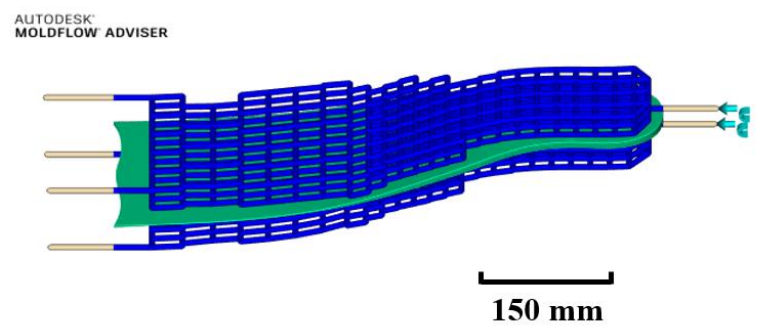

(b) Conventional CPS

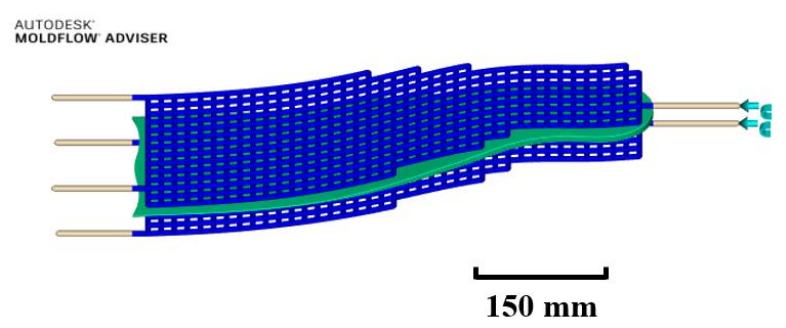

Figure 6-12. Machine learning aided CPS and conventional CPS 
(a) Temperature distribution: machine learning aided CPS

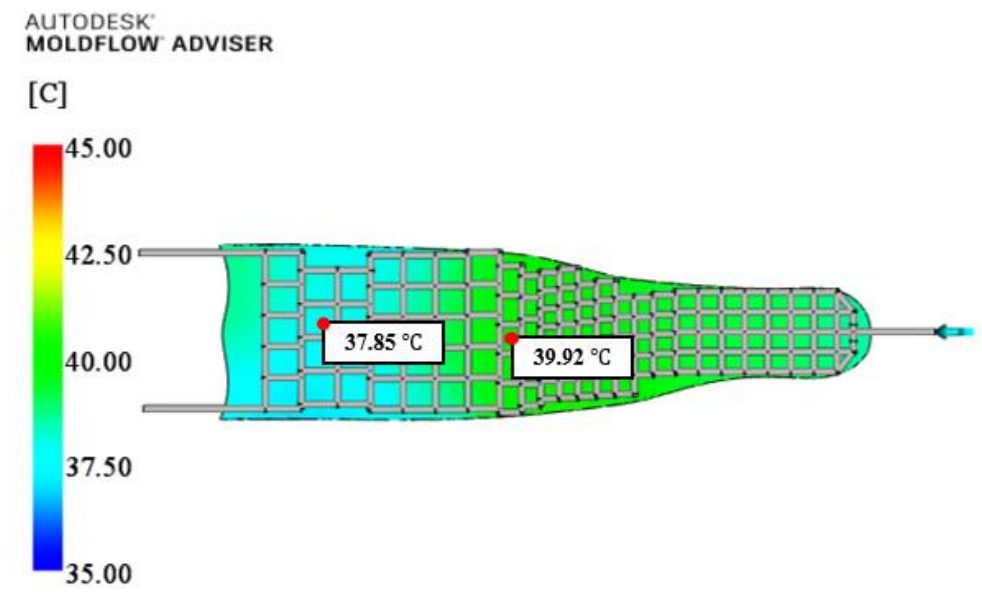

(b) Temperature distribution: conventional CPS

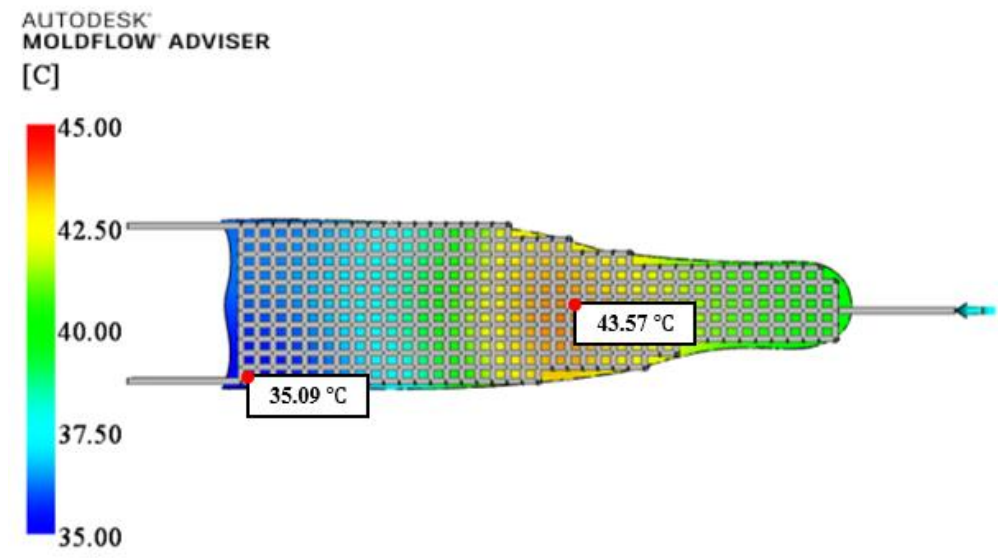

Figure 6-13. Resulting part temperature distribution for machine learning aided CPS and conventional CPS

To further compare evaluate the temperature variance of the part produced by machine learning aided and conventional CPS, the temperature variance distribution is calculated along the part surface in Figure 6-14. Comparing to an approximately $-4{ }^{\circ} \mathrm{C}$ variance at the thinner regions and a $4{ }^{\circ} \mathrm{C}$ variance at the thicker regions for conventional CPS, a close to zero temperature variance is found for most of the part regions for machine learning aided CPS. These results indicate that the machine learning aided CPS is able to achieve a significantly reduced part temperature variance 
compared to conventional CPS due to the fact that it not only conforms to the part geometry but also the part thickness distributions.

(a) Temperature variance: machine learning aided CPS

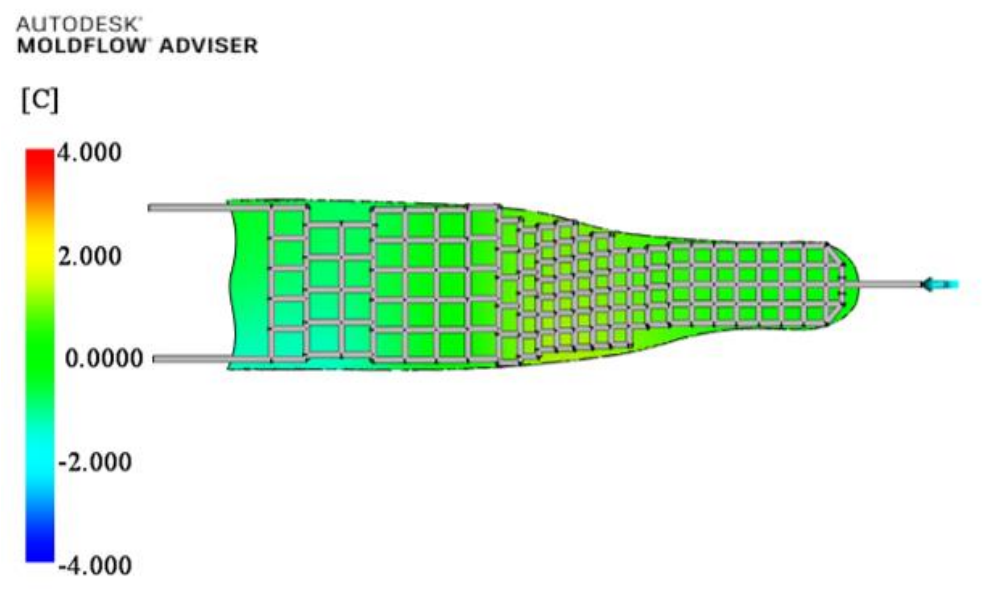

(b) Temperature variance: conventional CPS

$$
\begin{aligned}
& \text { AUTODESK } \\
& \text { MOLDFLOW ADVISER }
\end{aligned}
$$

[C]

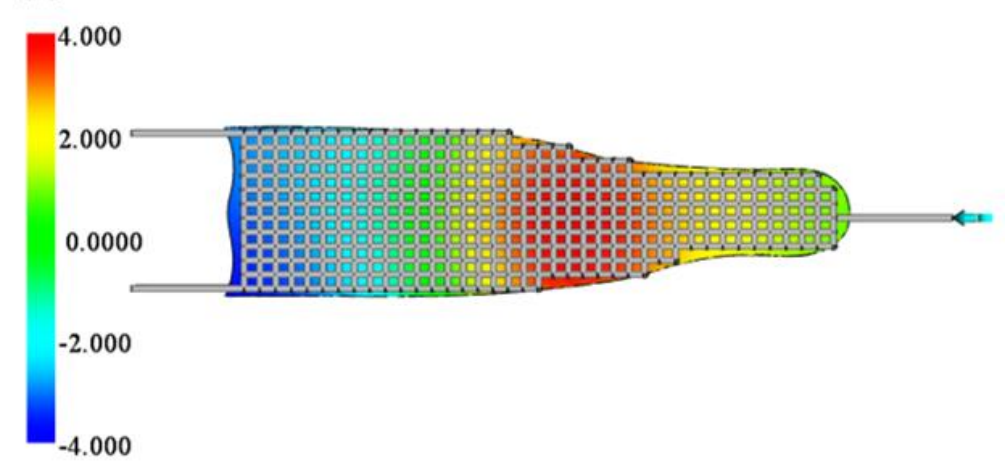

Figure 6-14. Resulting part temperature variance for machine learning aided CPS and conventional CPS

\subsection{Implementation of MLACCD software}

To implement the algorithms of MLACCD method proposed in this work, a MLACCD software is designed with a user-friendly GUI. With this software, the user will be able to obtain meshes of MLACCD channels based on their desired part design, conformal cooling topology, and target temperature. The MLACCD method will generate suitable cooling channels that are able to cool 
the part and achieve a highly uniform part surface temperature that is closest to a predefined target temperature. In this section, the main functions of the MLACCD software are introduced, whereas example cooling surfaces are given to further illustrate the expected outputs from the software.

\subsubsection{Software implementation}

The MLACCD software is designed based on the python tkinter GUI library, the pre-designed models that has the MLACCD implementation, and a data base composed of machine learning

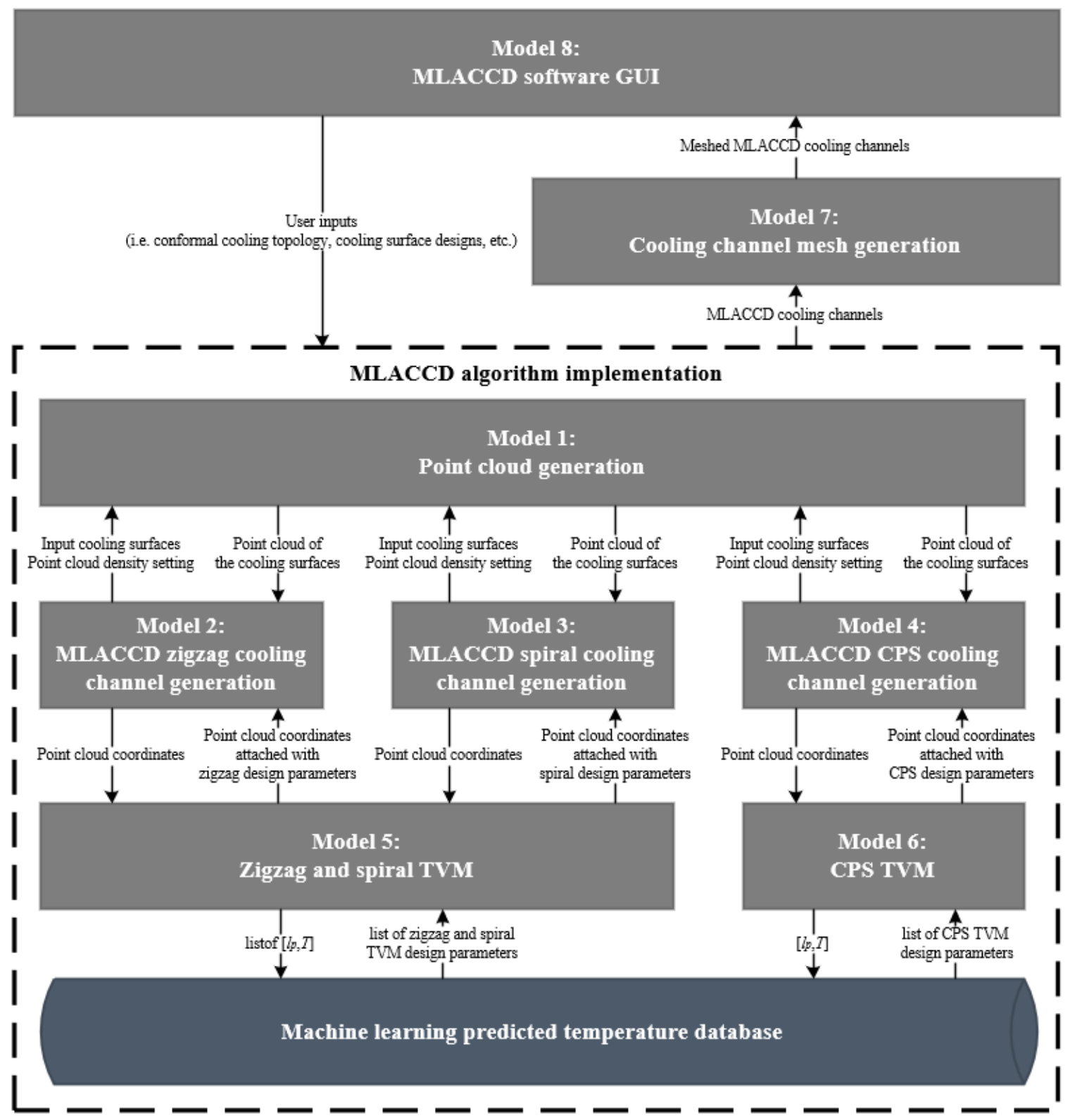

Figure 6-15. MLACCD software model relations 
predicted temperature. The relations between the models are shown in Figure 6-15. Basically, functions within the Model 1 to Model 7 are called by the Model 8 (GUI model) so that the interactions between the user and the MLACCD programs can be achieved. Specifically, these MLACCD algorithms are integrated by the GUI model with a proper calling sequence, so that the corresponding graphical interfaces will be displayed once the user interacts with the software. To improve the maintainability of the software, the software models are designed with high cohesion (the functions within the same model are built to achieve a common goal), and low coupling (unnecessary relations between models are removed).

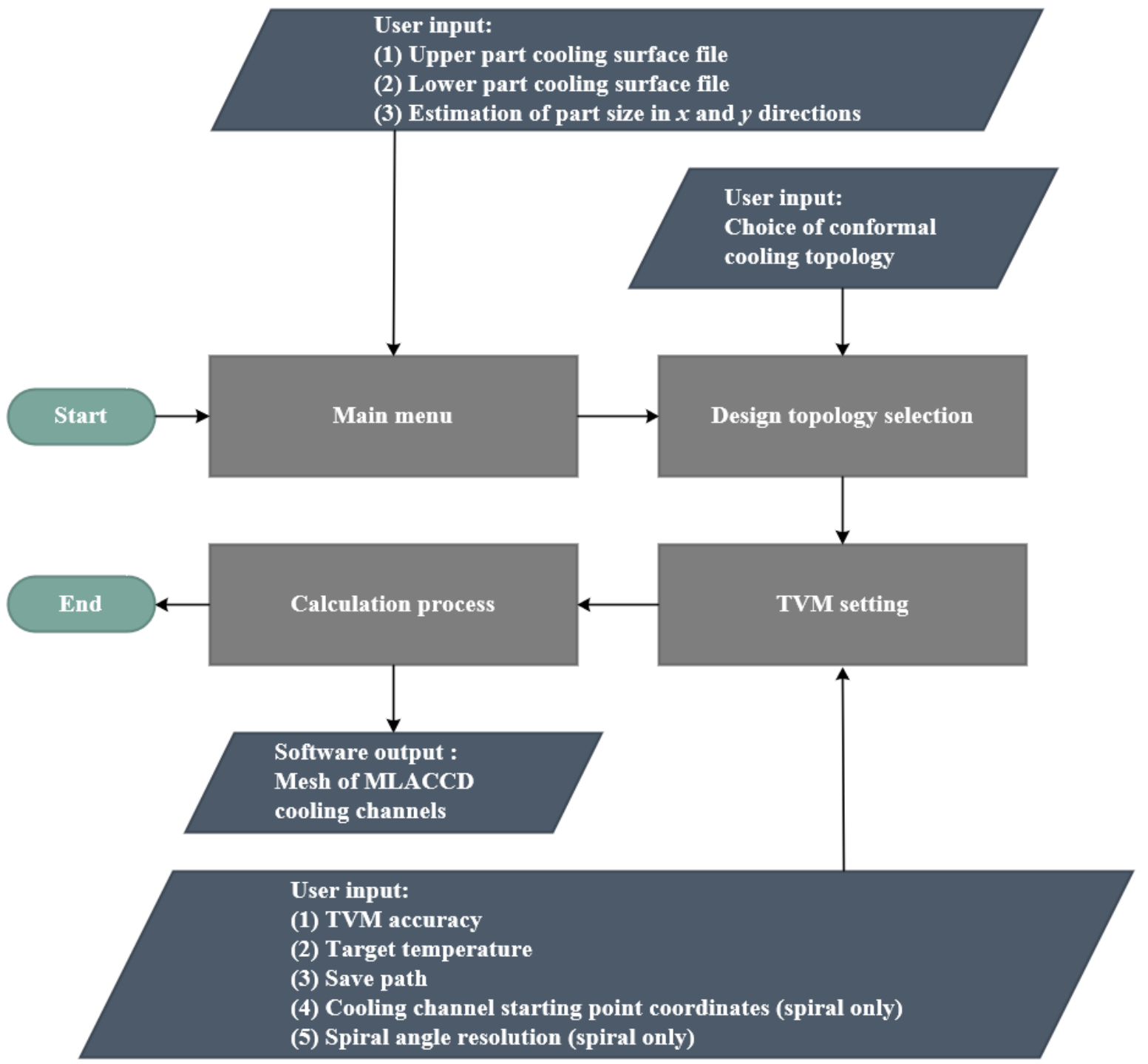

Figure 6-16. MLACCD software flowchart 
The flowchart of the software is shown in Figure 6-16, where are four steps for the software to generate the meshed MLACCD channels for the user. The main menu GUI is shown in Figure 6-17, where the user is asked to type in the file directories for the upper and lower cooling surface of the part, and the estimations of the part size in $x$ and $y$ directions. To explain, the file directories are used to upload the cooling surfaces from specified locations for analysis, and the sizes of the part are used in the calculation of the point cloud density, which will be discussed later.

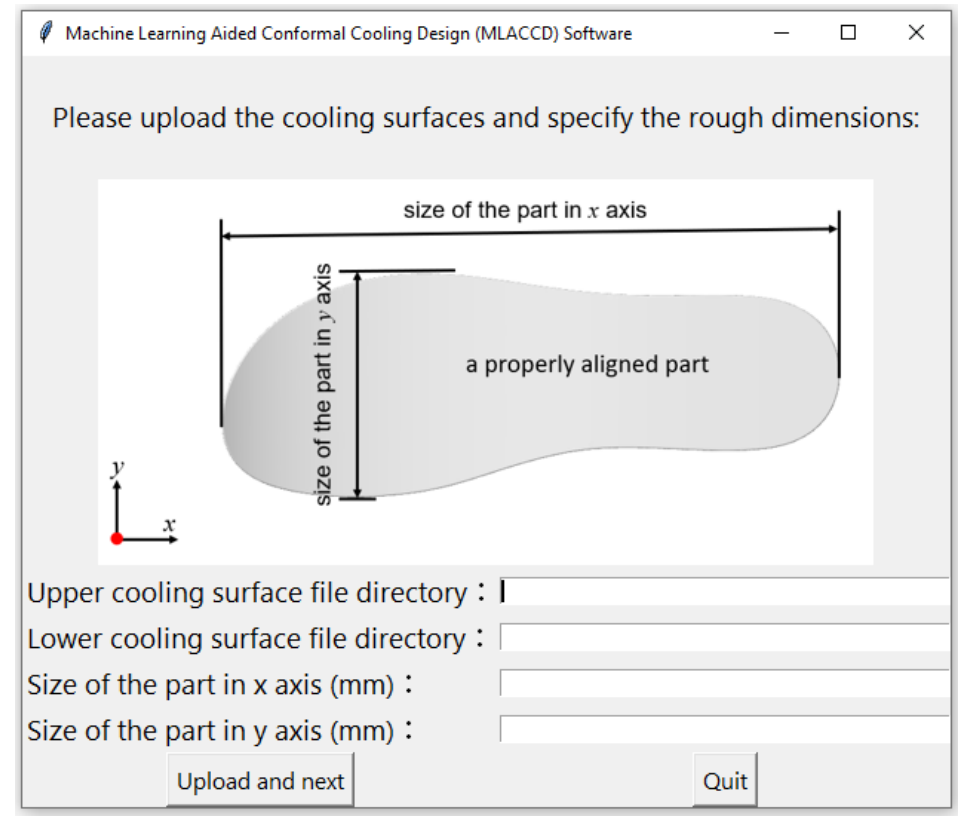

Figure 6-17. MLACCD software main menu

By clicking the "Upload and next" button, the software navigates to the design topology selection menu as shown in Figure 6-18. In this menu, three conformal cooling topologies are provided, where the topology that user prefers can be selected for TVM analysis. The porous cooling channels are not implemented in this interface, since CPS is able to provide a better cooling performance and a smaller pressure drop as a special type among different porous cooling systems [13]. 


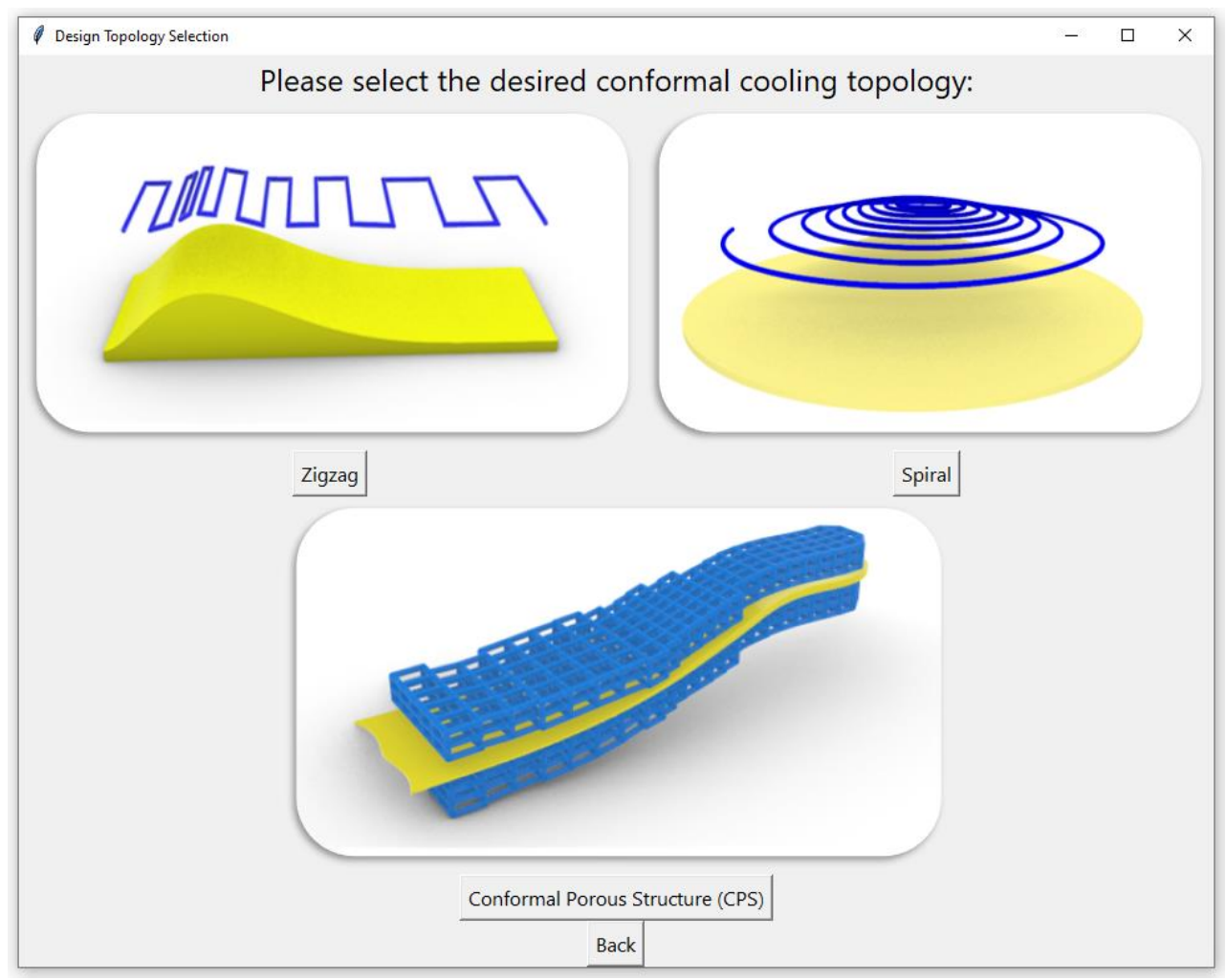

Figure 6-18. Design topology selection menu

After the cooling topology is selected, the TVM setting menu is shown is acquire the inputs including the TVM accuracy, save path, and the target temperature from the user as shown in Figure 6-19. For spiral cooling channels TVM setting as shown in Figure 6-19a, additional user inputs such as the spiral angle resolution $N$, and the cooling channel start point coordinates are required to fully constrain the control lines of the cooling channels. The TVM accuracy together with the size of the part reflects the density of the point cloud during the analysis as shown in equation (6-1):

$$
\rho_{\text {point cloud }}=\frac{\text { TVM accuracy } \times \text { global accuracy }}{s_{x} s_{y}}
$$

where $s_{x}, s_{y}$ are the estimate size of the part in $x$ and $y$ directions, $\rho_{\text {point cloud }}$ is the point cloud density. The global accuracy is implemented as 500 inside the current version of the software to 
achieve a simulation time ranging from roughly 5 minutes to 10 hours according to different TVM accuracy defined by the user.

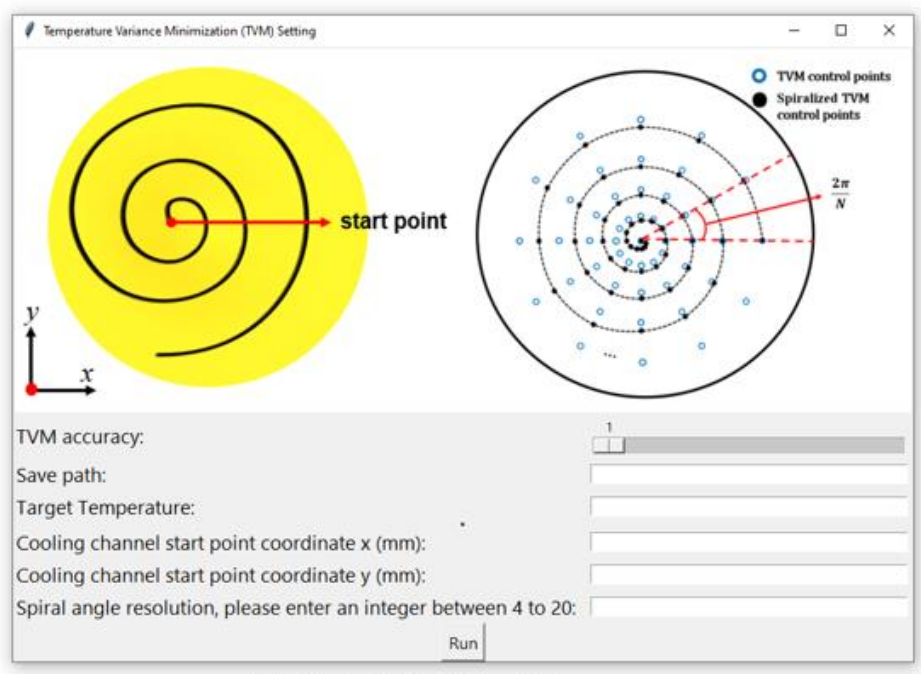

(a) Spiral TVM setting menu

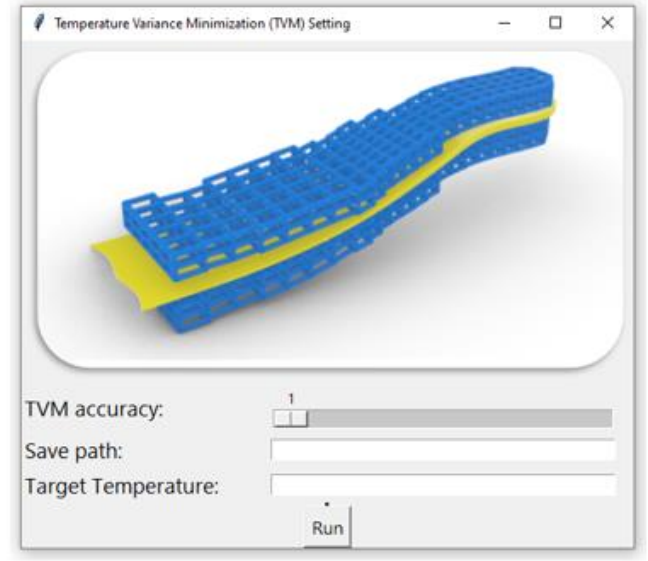

(b) CPS TVM setting menu

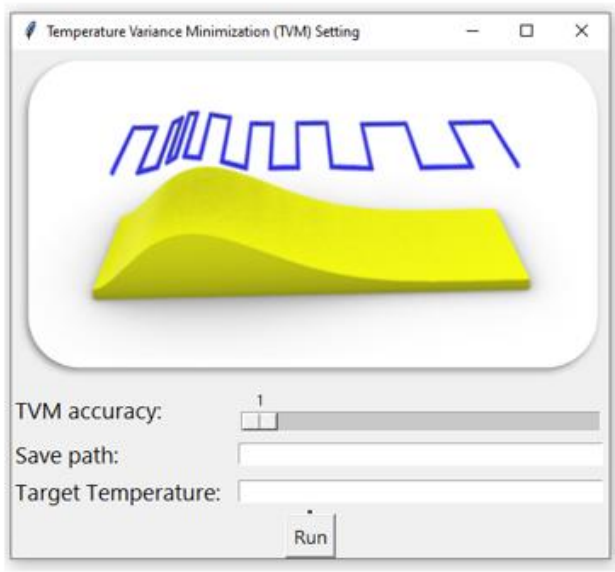

(c) Zigzag TVM setting menu

Figure 6-19. Temperature variance minimization (TVM) setting menu

Figure 6-20 shows the window that appears once the TVM setting is completed and the calculation process starts. The software displays a program dialog box and a process bar to provide the information about the current objective and progress of the calculation and potential warning messages for the user. Once the calculation is finished, the meshed MLACCD channels will be saved to the save path, where the supported input and output format of the current version software is STL. 


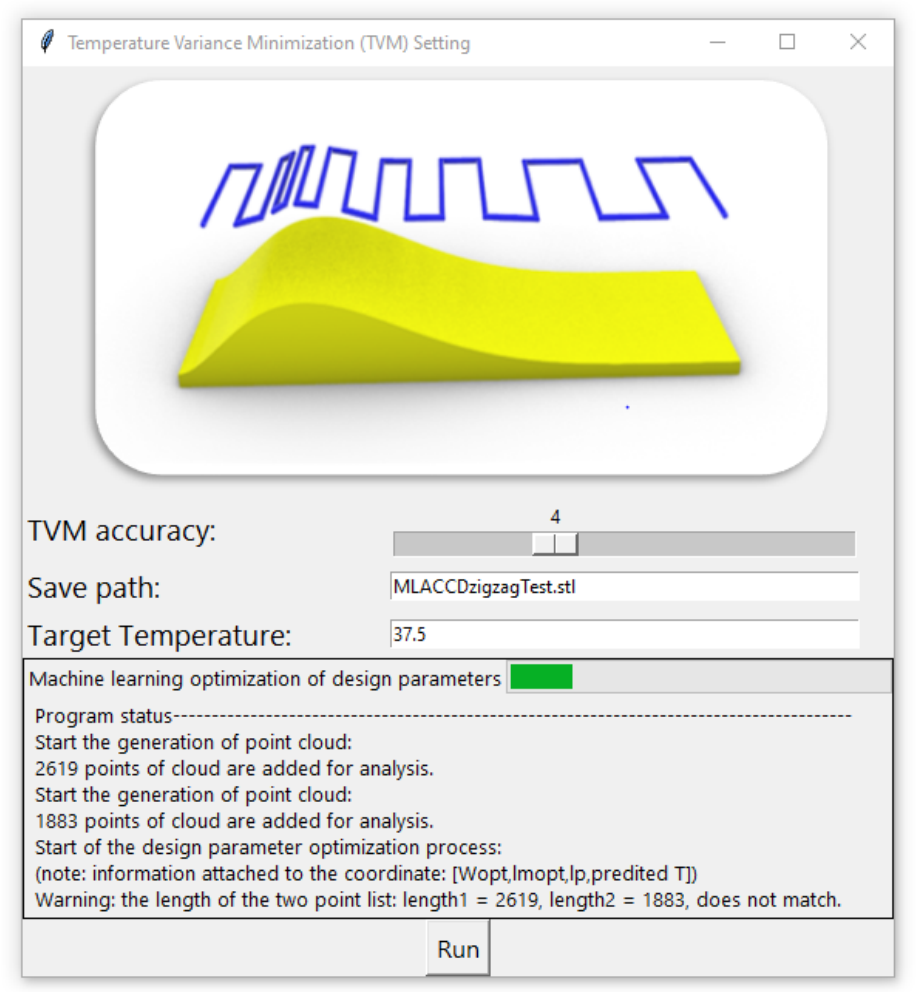

Figure 6-20. MLACCD program dialog window

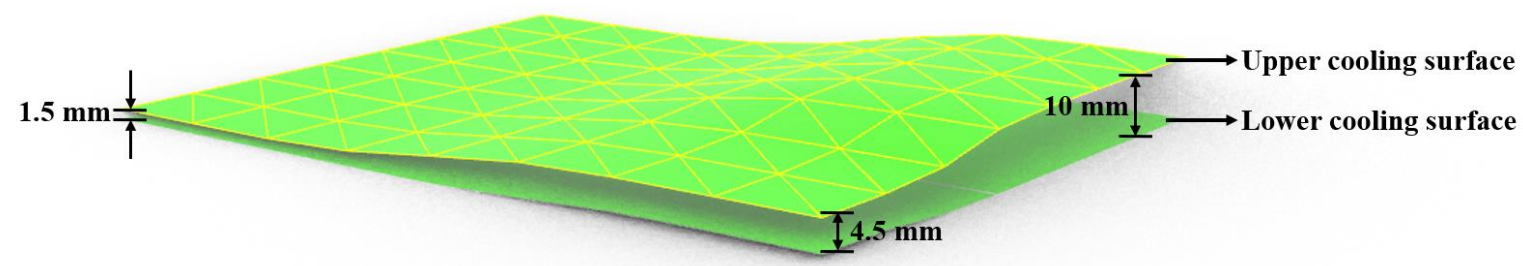

Figure 6-21. Example cooling surfaces

To illustrate the expected output of the software, example cooling surfaces as shown in Figure 6-21 are provided, where this test part has a highly non-uniform thickness values distributed long its surface. Following the instructions of the software as shown in Figure 6-17 to Figure 6-20, the mesh of example MLACCD channels can be generated, where the CPS is selected for these cooling surfaces for illustration purposes. The MLACCD CPS cooling channels generated by the software are shown in Figure 6-22, which has optimized cell size and pitch to part surface distance 
distributed along the cooling surface of the part. Based on the mesh of the MLACCD channels, the user can either make the MLACCD wireframe re-built for simulation purposes or generate the final mold design using the mesh Boolean subtraction between the mold object and the mesh of the MLACCD channels.

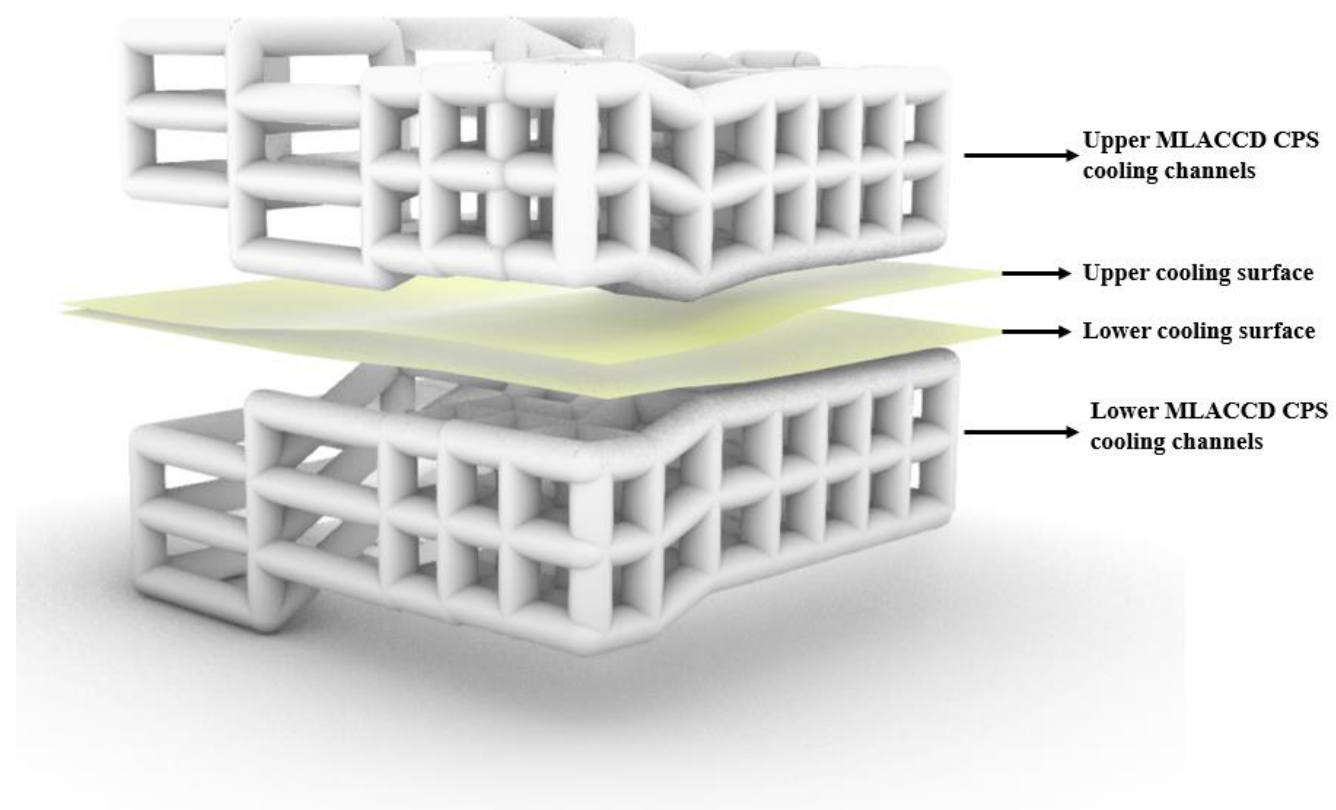

Figure 6-22. MLACCD CPS cooling channels for example cooling surfaces

Exceptional operations are required to generate the MLACCD cooling channels for the parts with special properties such as the one with large geometrical angle variations as shown in Figure 6-23, or the one containing very small geometrical features as shown in Figure 6-24. For the parts with large geometrical angle variations as shown in Figure 6-23a, the total cooling surface can be divided into different cooling regions. Specifically, these cooling regions are separated by the cooling surface division lines, which are the edges of two neighboring surfaces with the angles that are close to 90 degrees. After the total cooling surface is divided into different cooling regions as shown in Figure 6-23b, the MLACCD cooling channels can be formed based on the cooling channels that are separately constructed for each cooling region as shown in Figure 6-23c. 


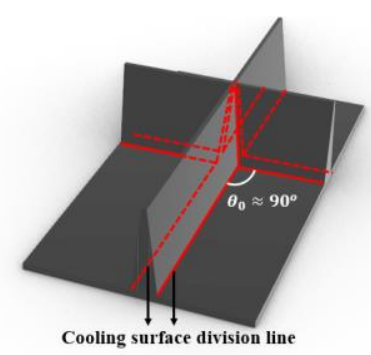

(a) Example part with large geometrical angle variations

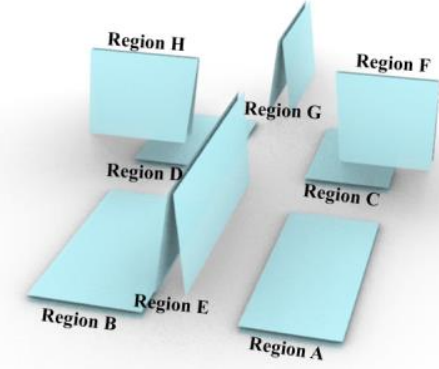

(b) Separated cooling regions

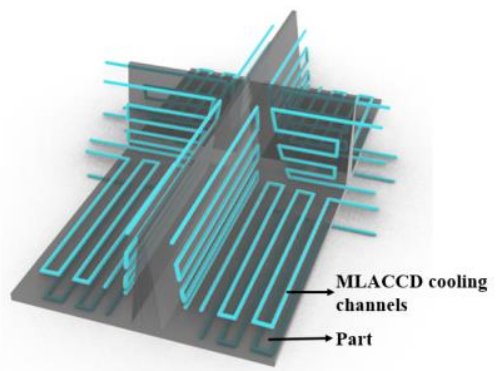

(c) MLACCD cooling channels for the part with large geometrical angle variations

Figure 6-23. Generation of cooling channels for the part with large geometrical angle variations

For the parts with very small geometrical features which have the feature size $d_{0}$ smaller than the minimum pitch width of the cooling channels as shown in Figure 6-24, the thickness-averaged cooling surfaces are required to generate the MLACCD cooling channels. The reason of calculating the thickness-averaged cooling surfaces is that the dimensions of those detailed features are too small for the cooling channels being conformal to them. For an example part with gear features as shown in Figure 6-24a, the distance between the two cooling surfaces would be the average thickness of the gear features calculated in Figure 6-24b. Based on the thicknessaveraged cooling surface, the MLACCD cooling channels for the part with small features can be generated as shown in Figure 6-24c.

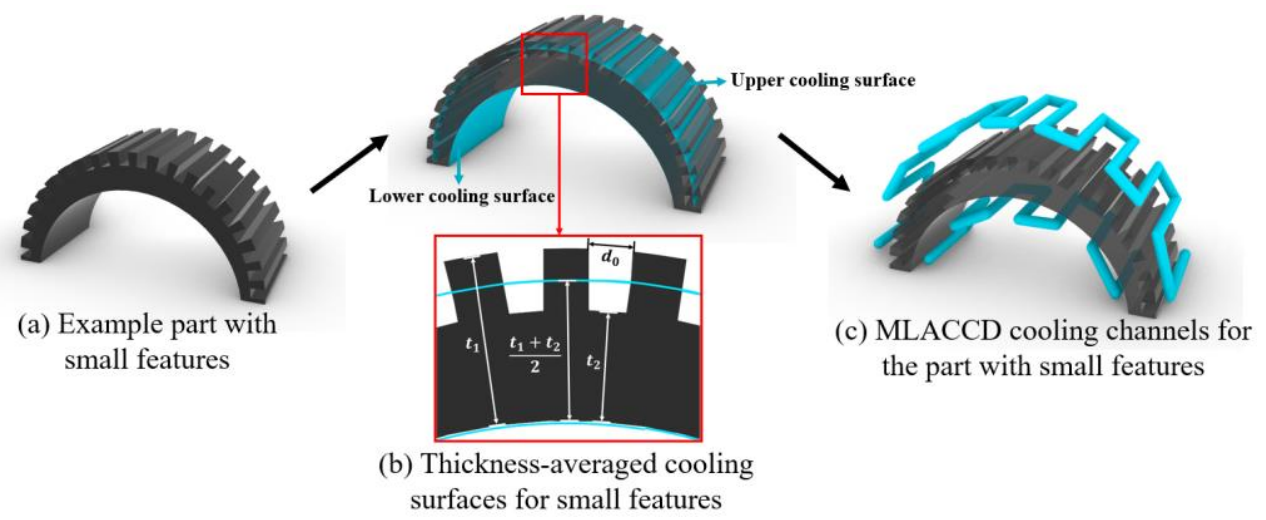

Figure 6-24. Generation of cooling channels for the part with small features 


\subsection{Summary}

In this chapter, three case studies including a freeform part surface, a shoe sole, and a swimming pedal are proposed to validate the effectiveness of the proposed MLACCD. The software implementation of the validated MLACCD method is also discussed. For comparison purpose, both the conventional conformal cooling design and the MLACCD are applied in each case study. The results indicate that the parts cooled by MLACCD have a significantly reduced temperature variance, which is due to the fact that the MLACCD channels are conformal not only to the part surface but also to the part thickness distributions. In addition, for the cooling topologies such as zigzag and spiral where the pressure drop is high for its original design, the MLACCD can provide a cooling system with a much lower coolant pressure drop. From the results of these case studies, it can be concluded that the MLACCD is a very successful conformal cooling design approach to solve the temperature variance problem in the plastic injection molding industry by making the cooling system smarter and more energy efficient. The MLACCD software are composed of six models containing the MLACCD algorithms, a meshing model, and a GUI model that achieves the interactions between the program and the user. Based on this software, the user can generate the MLACCD channels with their desired part cooling surfaces, target temperature, and cooling channel topologies. 


\section{CHAPTER 7}

\section{Conclusions and future works}

Injection molding is a common plastic manufacturing process which fabricates parts by injecting molten materials into the mold. Although the cooling process represents the largest portion of the injection cycle time, the cooling performance of the traditional cooling channels is limited by the conventional drilling and casting process. However, advanced AM technology liberates the possibilities for designing and manufacturing conformal cooling channels that could achieve a uniform pitch to mold surface distance. Nevertheless, this traditional conformal cooling design concept could result in a large temperature variance and a poor part quality, since it delivers a uniformly distributed cooling efficiency even for the part regions with different thickness values and thermal inertia. Thus, the temperature variance of the conformal cooling channels caused by varied part thickness remains unsolved. Due to the theoretical complexity and potential inaccuracy for predicting the part surface temperature from conformal cooling design parameters and the part thickness values, the part surface temperature cannot be efficiently derived using the existing conformal cooling heat transfer theories. To effectively predict the part surface temperature and eliminate the resulting part temperature variance, this thesis presents a novel machine learning aided design of conformal cooling channels, where in total four conformal cooling topologies are chosen to validate the proposal design method.

The proposed a novel conformal cooling design method generates cooling channels that are conformal not only to the part surface, but also to the part thickness distributions. The contributions of this research are listed as following:

\section{Development of a machine learning surrogate temperature prediction model.}

With more than 1000 sets of the training data being imported into a two-layer ANN, a machine learning surrogate temperature prediction model is derived. To explain, the 
design parameters are first identified from given cooling topologies following three principles: (1) the design parameters should have the ability to effectively affect the cooling efficiency of the cooling system; (2) it should be practical to adjust the design parameters in the optimization process; (3) the design parameters should be essential in fully defining the geometrical design of the cooling channels. Based on the identified design parameters, the training data is generated and collected through the Moldflow Advisor ${ }^{\circledR}$ cooling simulation and fed into a two-layer ANN. As a result, a surrogate temperature prediction model is obtained. The loss function during the training process represents a steady decrease, and the maximum model error is less than $0.5^{\circ} \mathrm{C}$. The local part surface temperature prediction time of the surrogate temperature model based on a given set of design parameters is less than 0.5 seconds. These evaluation results indicate that a fast and accurate prediction of part surface temperature is achieved through the supervised machine learning.

\section{Development of TVM algorithms for major conformal cooling topologies.}

In the TVM tool, different optimization strategies are designed for zigzag, spiral, porous, and CPS cooling channels to minimize the part surface temperature variance based on a given $\mathrm{CAD}$ design of the part. The surrogate machine learning temperature prediction model is continuously applied to predict the part temperature during the optimization process. In addition, the design parameters of zigzag, spiral porous, and CPS cooling channels are updated to minimize the temperature variance of the part based on the machine learning surrogate temperature prediction model. Due to the large design variance of different cooling topologies, different optimization strategy is proposed for each topological design of conformal cooling channels, which is carefully discussed in Chapter 
5. As a result, innovative MLACCD channels can be created to provide a smarter cooling without a large temperature variance resulted on the part surface. The effectiveness of the MLACCD is validated through three cases studies including a freeform part surface, a shoe sole, and a swimming pedal. Overall, a $17 \%$ to $93 \%$ reduction of the temperature variance is achieved. For the conformal cooling topologies that have high pressure drop such as the zigzag and spiral cooling channels, a significant reduction of the pressure drop is also achieved due to a decreased length of the cooling channels.

\section{Implementation of MLACCD software.}

Based on the TVM algorithms and the machine learning surrogate temperature prediction model, the MLACCD software is implemented to generate the MLACCD channels from the user defined part cooling surfaces and target temperature. This software provides a user-friendly interface that guide the researchers and industrial users to create their own MLACCD channels with desired conformal cooling topologies, which is discussed in Chapter 6.

The outcome of this research is an innovative conformal cooling design method which can effectively reduce the part temperature variance and improve the energy efficiency of the mold.

Some future works to continue improve the proposed MLACCD method is listed here. First, the length of the cooling channels could be considered as another design parameter, and be imported into the supervised machine learning algorithm to improve the performance of the surrogate model. The coolant typically exhibits a temperature rise from the inlets to the outlets for the cooling channels with a large length. This temperature rise will introduce a reduction in temperature prediction accuracy since the current model treats the temperature of the coolant as a constant value. 
Second, it is interesting to experimentally validate the effectiveness of the MLACCD in the future. Although the accuracy of the Moldflow Advisor ${ }^{\circledR}$ simulation software is proofed by previous publications [14-16, 24], it is still worthwhile to investigate the performance of the MLACCD mold on a real injection molding machine. The following steps could be the potential procedures for future experiments: (1) fabricate a MLACCD mold and a CCCD mold via AM with H13 tool steel with embedded sensor based on a selected case study and channel topology (i.e. shoe sole case study, zigzag cooling topology); (2) manufacture the parts with the printed molds on an industrialized injection molding machine until the temperature cycle of the mold become stable; (3) capture the thermal images of the parts fabricated by the MLACCD mold and CCCD mold right after it is ejected, record the part temperature distributions; (4) compare the experimental data and the numerical simulation results.

In addition, the manufacturability of this machine learning aided design of conformal cooling is not analyzed due to the challenges of printing the potential sharp corners of cooling channels. Thus, comparisons could be made between different machine learning aided optimization strategies (i.e. update priority of the design parameters) in terms of their resulting manufacturability. This manufacturability analysis can be incorporated into the cooling channel optimization method to achieve a user-preferred balance of the manufacturability and the cooling performance of conformal cooling channels.

Furthermore, the proposed MLACCD method can be applied to aid other types of injection molding process such as the resin transfer molding (RTM) for composite materials, where the shrinkage of the part may occur due to the temperature variance issue [48]. Specifically, additional training data could be constructed based on the method proposed in Chapter 4 for the composite parts fabricated via RTM process. These training data can be fed into the ANN so that the 
MLACCD will be able to support the temperature variance minimization of the composites parts manufactured by RTM, which can further benefit the aerospace and automotive industries where the composite materials have great application potentials $[49,50]$.

Finally, new topological designs of the conformal cooling channels may appear in the future. Utilizing the advantages of machine learning, the proposed method can be rapidly applied to these new topologies so that the uniformity of resulting part temperature distribution can be greatly improved. 


\section{REFERENCE}

[1] S. Kalpakjian, K. Vijai Sekar, and S. R. Schmid, Manufacturing engineering and technology. Pearson, 2014.

[2] D. V. Rosato and M. G. Rosato, Injection molding handbook. Springer Science \& Business Media, 2012.

[3] E. A. Ott and M. W. Peretti, "Metal injection molding of alloy 718 for aerospace applications," Jom, vol. 64, no. 2, pp. 252-256, 2012.

[4] J. Holbery and D. Houston, "Natural-fiber-reinforced polymer composites in automotive applications," Jom, vol. 58, no. 11, pp. 80-86, 2006.

[5] R. Ruprecht, T. Gietzelt, K. Müller, V. Piotter, and J. Haußelt, "Injection molding of microstructured components from plastics, metals and ceramics," Microsystem Technologies, vol. 8, no. 4-5, pp. 351-358, 2002.

[6] J. Teel. "Introduction to Injection Molding." https://predictabledesigns.com/introductionto-injection-molding/ (accessed Feburary, 2020).

[7] D. Yao and B. Kim, "Development of rapid heating and cooling systems for injection molding applications," Polymer Engineering and Science, vol. 42, no. 12, pp. 2471-2481, 2002.

[8] G. Venkatesh, Y. R. Kumar, and G. Raghavendra, "Comparison of straight line to conformal cooling channel in injection molding," Materials Today: Proceedings, vol. 4, no. 2, pp. 1167-1173, 2017.

[9] S. B. M. Yasin, N. F. M. Sani, S. Adnan, Z. Zulkifli, Z. Ahmad, and S. N. S. Ismail, "Efficiency Cooling Channel at Core Side Incorporating with Baffle and Bubbler System," in Proceedings of the Second International Conference on the Future of ASEAN (ICoFA) 2017-Volume 2, 2018: Springer, pp. 515-522.

[10] X. Xu, E. Sachs, and S. Allen, "The design of conformal cooling channels in injection molding tooling," Polymer Engineering and Science, vol. 41, no. 7, pp. 1265-1279, 2001.

[11] S. Ford and M. Despeisse, "Additive manufacturing and sustainability: an exploratory study of the advantages and challenges," Journal of Cleaner Production, vol. 137, pp. 1573-1587, 2016.

[12] E. Sachs, E. Wylonis, S. Allen, M. Cima, and H. Guo, "Production of injection molding tooling with conformal cooling channels using the three dimensional printing process," Polymer Engineering \& Science, vol. 40, no. 5, pp. 1232-1247, 2000.

[13] Y. Tang, Z. Gao, and Y. F. Zhao, "Design of conformal porous structures for the cooling system of an injection mold fabricated by Additive Manufacturing Process," Journal of Mechnical Design, vol. 141, no. 10, pp. 1-22, 2019.

[14] Y. Wang, K.-M. Yu, and C. C. Wang, "Spiral and conformal cooling in plastic injection molding," Computer-Aided Design, vol. 63, pp. 1-11, 2015.

[15] Y. Wang, K.-M. Yu, C. C. Wang, and Y. Zhang, "Automatic design of conformal cooling circuits for rapid tooling," Computer-Aided Design, vol. 43, no. 8, pp. 1001-1010, 2011.

[16] H.-S. Park and N. H. Pham, "Design of conformal cooling channels for an automotive part," International Journal of Automotive Technology, vol. 10, no. 1, pp. 87-93, 2009.

[17] M. Khan, S. K. Afaq, N. U. Khan, and S. Ahmad, "Cycle time reduction in injection molding process by selection of robust cooling channel design," ISRN Mechanical Engineering, vol. 2014, pp. 1-8, 2014. 
[18] K. Au and K. Yu, "A scaffolding architecture for conformal cooling design in rapid plastic injection moulding," The International Journal of Advanced Manufacturing Technology, vol. 34, no. 5-6, pp. 496-515, 2007.

[19] T. Wu, K. Liu, and A. Tovar, "Multiphase topology optimization of lattice injection molds," Computers and Structures, vol. 192, pp. 71-82, 2017.

[20] L. Crema, M. Sorgato, and G. Lucchetta, "Thermal optimization of deterministic porous mold inserts for rapid heat cycle molding," International Journal of Heat and Mass Transfer, vol. 109, pp. 462-469, 2017.

[21] K. Au and K. Yu, "Modeling of multi-connected porous passageway for mould cooling," Computer-Aided Design, vol. 43, no. 8, pp. 989-1000, 2011.

[22] H. Brooks and K. Brigden, "Design of conformal cooling layers with self-supporting lattices for additively manufactured tooling," Additive Manufacturing, vol. 11, pp. 16-22, 2016.

[23] C. Li, "A feature-based approach to injection mould cooling system design," ComputerAided Design, vol. 33, no. 14, pp. 1073-1090, 2001.

[24] H.-S. Park and X.-P. Dang, "Optimization of conformal cooling channels with array of baffles for plastic injection mold," International Journal of Precision Engineering Manufacturing, vol. 11, no. 6, pp. 879-890, 2010.

[25] S. A. Jahan et al., "Implementation of conformal cooling \& topology optimization in 3D printed stainless steel porous structure injection molds," Procedia Manufacturing, vol. 5, pp. 901-915, 2016.

[26] S. Jahan and H. El-Mounayri, "A Thermomechanical Analysis of Conformal Cooling Channels in 3D Printed Plastic Injection Molds," Applied Science, vol. 8, no. 12, p. 2567, 2018.

[27] S. A. Jahan, T. Wu, Y. Zhang, J. Zhang, A. Tovar, and H. El-Mounayri, "Effect of Porosity on Thermal Performance of Plastic Injection Molds Based on Experimental and Numerically Derived Material Properties," in Mechanics of Additive and Advanced Manufacturing, Volume 9: Springer, 2018, pp. 55-63.

[28] I. Pandelidis and Q. Zou, "Optimization of injection molding design. Part I: Gate location optimization," Polymer Engineering and Science, vol. 30, no. 15, pp. 873-882, 1990.

[29] B. Lee and B. Kim, "Optimization of part wall thicknesses to reduce warpage of injection-molded parts based on the modified complex method," Polymer-plastics Technology and Engineering, vol. 34, no. 5, pp. 793-811, 1995.

[30] D. Dimla, M. Camilotto, and F. J. J. o. M. P. T. Miani, "Design and optimisation of conformal cooling channels in injection moulding tools," vol. 164, pp. 1294-1300, 2005.

[31] S. A. Jahan, W. Tong, Z. Yi, Z. Jing, and H. Elmounayri, "Thermo-mechanical Design Optimization of Conformal Cooling Channels using Design of Experiments Approach," Procedia Manufacturing, vol. 10, pp. 898-911, 2017.

[32] M. et al., "Warpage optimisation on the moulded part with conformal cooling channels using response surface methodology (RSM) and genetic algorithm (GA) optimisation approaches."

[33] S. Kitayama, H. Miyakawa, M. Takano, and S. Aiba, "Multi-objective optimization of injection molding process parameters for short cycle time and warpage reduction using conformal cooling channel," International Journal of Advanced Manufacturing Technology, vol. 88, no. 5-8, pp. 1735-1744. 
[34] M. S. Jacques, "An analysis of thermal warpage in injection molded flat parts due to unbalanced cooling," Polymer Engineering and Science, vol. 22, no. 4, pp. 241-247, 1982.

[35] Z. Shayfull, M. Fathullah, S. Sharif, S. Nasir, and N. Shuaib, "Warpage Analysis on Ultra-Thin Shell by Using Taguchi Method and Analysis of Variance(ANOVA) for Three-Plate Mold," International Review of Mechanical Engineering, vol. 5, no. 6, pp. 1116-1124, 2011.

[36] B. Lee and B. Kim, "Variation of part wall thicknesses to reduce warpage of injectionmolded part: robust design against process variability," Polymer-Plastics Technology and Engineering, vol. 36, no. 5, pp. 791-807, 1997.

[37] T. Erzurumlu and B. Ozcelik, "Minimization of warpage and sink index in injectionmolded thermoplastic parts using Taguchi optimization method," Materials \& design, vol. 27, no. 10, pp. 853-861, 2006.

[38] A. Ahmad et al., "Optimization of warpage defect in injection moulding process using ABS material," in 2009 Third Asia International Conference on Modelling \& Simulation, 2009: IEEE, pp. 470-474.

[39] K. P. Murphy, Machine learning: a probabilistic perspective. MIT press, 2012.

[40] D. Michie, D. J. Spiegelhalter, and C. Taylor, "Machine learning," Neural and Statistical Classification, vol. 13, no. 1994, pp. 1-298, 1994.

[41] L. Monostori, "AI and machine learning techniques for managing complexity, changes and uncertainties in manufacturing," Engineering Applications of Artificial Intelligence, vol. 16, no. 4, pp. 277-291.

[42] P. Priore, D. d. l. Fuente, J. Puente, and J. Parre?o, "A comparison of machine-learning algorithms for dynamic scheduling of flexible manufacturing systems," Engineering Applications of Artificial Intelligence, vol. 19, no. 3, pp. 247-255.

[43] H. M. Chi et al., "Machine learning and genetic algorithms in pharmaceutical development and manufacturing processes," Decision Support Systems, vol. 48, no. 1, pp. 69-80, 2009.

[44] P. Priore, D. DE LA FUENTE, A. GOMEZ, and J. PUENTE, "A review of machine learning in dynamic scheduling of flexible manufacturing systems," Artificial Intelligence for Engineering Design Analysis \& Manufacturing, vol. 15, no. 3, pp. 251-263.

[45] H. S. Park, D. X. Phuong, and S. Kumar, "AI Based Injection Molding Process for Consistent Product Quality," Procedia Manufacturing, vol. 28, pp. 102-106, 2019.

[46] H. Shi, S. Xie, and X. Wang, "A warpage optimization method for injection molding using artificial neural network with parametric sampling evaluation strategy," The International Journal of Advanced Manufacturing Technology, vol. 65, no. 1-4, pp. 343353, 2013.

[47] S. Haykin, Neural networks: a comprehensive foundation. Prentice Hall PTR, 1994.

[48] M. Haider, P. Hubert, and L. Lessard, "Cure shrinkage characterization and modeling of a polyester resin containing low profile additives," Composites Part A: Applied Science and Manufacturing, vol. 38, no. 3, pp. 994-1009, 2007.

[49] A. Quilter, "Composites in aerospace applications," IHS White Paper, vol. 444, no. 1, 2001.

[50] G. Mahajan and V. Aher, "Composite material: a review over current development and automotive application," International journal of scientific and research publications, vol. 2, no. 11, pp. 1-5, 2012. 


\section{LIST OF PUBLICATIONS}

[1] Z. Gao, G. Dong, Y. Tang, Y. Zhao. Machine learning aided design of conformal cooling channels for injection molding. ASME. Journal of Mechanical Design. (In Press)

[2] Y. Tang, Z. Gao, Y. Zhao. Design of conformal porous structures for the cooling system of an injection mold fabricated by Additive Manufacturing Process. ASME. Journal of Mechanical Design 2019; 1-22. doi: 10.1115/1.4043680.

[3] Z. Gao, Y. Zhao. Machine learning aided design and optimization of conformal porous structure. IDETC-CIE 2020.

[4] Z. Gao, Y. Zhao. Machine learning aided optimization of conformal cooling channels for injection molding. HI-AM Conference 2019. 